\title{
CONFORMALLY COVARIANT BOUNDARY CORRELATION FUNCTIONS WITH A QUANTUM GROUP
}

\author{
KALLE KYTÖLÄ \\ kalle.kytola@aalto.fi \\ Department of Mathematics and Systems Analysis \\ P.O. Box 11100, FI-00076 Aalto University, Finland \\ Evelina Peltola \\ eveliina.peltola@unige.ch \\ Section de Mathématiques, Université de Genève, \\ 2-4 rue du Lièvre, C.P. 64, 1211 Genève 4, Switzerland
}

\begin{abstract}
Particular boundary correlation functions of conformal field theory are needed to answer some questions related to random conformally invariant curves known as Schramm-Loewner evolutions (SLE). In this article, we introduce a correspondence and establish its fundamental properties, which are used in the companion articles [JJK16, KP16] for explicitly solving two such problems. The correspondence associates Coulomb gas type integrals to vectors in a tensor product representation of a quantum group, a $q$-deformation of the Lie algebra $\mathfrak{s l}_{2}$. We show that desired properties of the functions are guaranteed by natural representation theoretical properties of the vectors.
\end{abstract}

\section{INTRODUCTION}

Boundary correlation functions in conformal field theories in general, and, in particular, questions about random conformally invariant curves, frequently lead to quite similar systems of partial differential equations whose boundary conditions are specified in terms of the asymptotic behavior of solutions. The main result of this article provides a systematic method to construct explicit solutions. The method is a form of the so-called "hidden quantum group symmetry of conformal field theories" FFK89, MR89, BMP90, GS90, FW91, PS91, RRRA91, Var92] (see also the textbooks [Fuc92, Var95, GRAS96]). In this article, we establish properties which are directly relevant for solving the PDE problems arising in the theory of Schramm-Loewner evolutions (SLE). In two companion articles, the results are applied to produce explicit answers to the questions of boundary visit probabilities of chordal SLEs [JJK16] and the pure geometries of multiple SLEs [KP16]. With appropriate modifications, the method can also be applied to bulk correlation functions - in [FP18b] it is applied to the construction of single-valued bulk correlation functions of conformal field theory.

At the heart of the method are integral solutions to differential equations, crucial to the entire Coulomb gas formalism of conformal field theory [DF85]. Such an idea was used already by Euler for solving the hypergeometric differential equation, and it can be summarized as follows. Let $\mathcal{D}$ be a differential operator acting in a variable $x$. Suppose that $f(x, w)$ is a function of $x$ and an auxiliary variable $w$ such 
that the differential operator $\mathcal{D}$ acting on $f$ gives an exact form in the $w$-variable: $(\mathcal{D} f) \mathrm{d} w=\mathrm{d} \eta$. Let $\Gamma$ be an integration surface in the $w$-variable, and define a function $F$ of $x$ by the integral

$$
F(x)=\int_{\Gamma} f(x, w) \mathrm{d} w .
$$

If the order of integration and differentiation can be exchanged in

$$
\mathcal{D} F=\mathcal{D} \int_{\Gamma} f \mathrm{~d} w=\int_{\Gamma}(\mathcal{D} f) \mathrm{d} w=\int_{\Gamma} \mathrm{d} \eta,
$$

then by Stokes' theorem we have

$$
\mathcal{D} F=\int_{\Gamma} \mathrm{d} \eta=\int_{\partial \Gamma} \eta
$$

If the surface $\Gamma$ is closed, $\partial \Gamma=\emptyset$, then the right hand side above vanishes, and $F$ therefore satisfies the differential equation $\mathcal{D} F=0$. When an appropriate auxiliary function $f$ is known, the remaining difficulty in solving the differential equation $\mathcal{D} F=0$ lies in choosing a surface $\Gamma$ which is not only closed, but also such that the constructed function $F=\int_{\Gamma} f \mathrm{~d} w$ satisfies whatever boundary conditions are imposed.

The method of this article exploits a hidden quantum group structure in the choice of the appropriate integration surface $\Gamma$ for solving certain partial differential equations of conformal field theory. We construct a linear correspondence from a representation of a quantum group to functions defined by integrals. In this correspondence, representation theoretic operations conveniently allow both to verify the closedness of the integration surface, and consequently the differential equations, and to analyze the boundary behavior of the function. The properties are formulated in a systematic and readily applicable fashion, as demonstrated in the examples provided later in this introduction.

1.1. Partial differential equations from conformal field theory. Let us parametrize the central charge $c$ of the conformal field theory by a number $\kappa>0$ via $c=c(\kappa)=13-\frac{3 \kappa}{2}-\frac{24}{\kappa}$. According to the seminal paper [BPZ84, the correlation functions of general fields can (usually) be reduced to correlation functions of the primary fields in the corresponding "conformal families". Moreover, when the conformal weights of the primary fields lie in the so-called Kac table, their correlation functions can be expected to satisfy partial differential equations, by virtue of degeneracies in the representation theory of the Virasoro algebra. For primary fields in the first row of the Kac table, the conformal weights are of the form

$$
h_{1, d}=\frac{(d-1)(2(d+1)-\kappa)}{2 \kappa}, \quad \text { with } d \in \mathbb{Z}_{>0},
$$

and explicit expressions for these partial differential equations have been found by Benoit and SaintAubin BSA88. These are the PDEs considered in the present article. Specifically, for a conformal field theory in the upper half-plane $\mathbb{H}=\{z \in \mathbb{C} \mid \Im \mathfrak{m}(z)>0\}$, a boundary correlation function of $n$ such primary fields is a function defined on the chamber

$$
\mathfrak{X}_{n}=\left\{\left(x_{1}, \ldots, x_{n}\right) \in \mathbb{R}^{n} \mid x_{1}<\cdots<x_{n}\right\},
$$

and if the fields at $x_{j}$, for $j=1, \ldots, n$, have conformal weights $h_{1, d_{j}}$, respectively, then the Benoit \& Saint-Aubin partial differential equations for the correlation function $F: \mathfrak{X}_{n} \rightarrow \mathbb{C}$ read

$$
\sum_{k=1}^{d_{j}} \sum_{\substack{p_{1}, \ldots, p_{k} \geq 1 \\ p_{1}+\cdots+p_{k}=d_{j}}} \frac{(-4 / \kappa)^{d_{j}-k}\left(d_{j}-1\right) !^{2}}{\prod_{u=1}^{k-1}\left(\sum_{i=1}^{u} p_{i}\right)\left(\sum_{i=u+1}^{k} p_{i}\right)} \times \mathcal{L}_{-p_{1}}^{(j)} \cdots \mathcal{L}_{-p_{k}}^{(j)} F\left(x_{1}, \ldots, x_{n}\right)=0,
$$

where $\mathcal{L}_{-p}^{(j)}=-\sum_{i \neq j}\left(\left(x_{i}-x_{j}\right)^{1-p} \frac{\partial}{\partial x_{i}}+(1-p) h_{1, d_{i}}\left(x_{i}-x_{j}\right)^{-p}\right)$. Moreover, covariance of the correlation function under global conformal transformations requires that under any Möbius transformation 
$\mu: \mathbb{H} \rightarrow \mathbb{H}$ of the upper half-plane such that $\mu\left(x_{1}\right)<\cdots<\mu\left(x_{n}\right)$, we have

$$
F\left(x_{1}, \ldots, x_{n}\right)=\prod_{i=1}^{n} \mu^{\prime}\left(x_{i}\right)^{h_{1, d_{i}}} \times F\left(\mu\left(x_{1}\right), \ldots, \mu\left(x_{n}\right)\right) .
$$

In the main result of the present article, we systematically construct integral solutions to the PDEs (1.3) and covariance condition 1.4 , and provide tools for analyzing their boundary conditions.

1.2. The role of the quantum group. The method we introduce in this article relies on the representation theory of the quantum group $\mathcal{U}_{q}\left(\mathfrak{s l}_{2}\right)$ in the generic, semisimple case (for the precise definitions, see Section 22. Informally, $\mathcal{U}_{q}\left(\mathfrak{s l}_{2}\right)$ is a deformation of the Lie algebra $\mathfrak{s l}_{2}$ of traceless complex $2 \times 2$ matrices, with a complex deformation parameter $q$ that we assume not to be a root of unity or zero. The deformation parameter is related to the central charge $c=c(\kappa)$ of the conformal field theory via $q=e^{\mathrm{i} \pi 4 / \kappa}$. Our assumption on $q$ corresponds to $\kappa \notin \mathbb{Q}$.

As an algebra, $\mathcal{U}_{q}\left(\mathfrak{s l}_{2}\right)$ is generated by an invertible Cartan element $K$, and raising and lowering operators $E$ and $F$, which shift the eigenvalues of $K$ by multiplicative factors $q^{2}$ and $q^{-2}$, respectively. To state our result, the following representation theoretical notions are needed. The algebra $\mathcal{U}_{q}\left(\mathfrak{s l}_{2}\right)$ has, for all positive integers $d$, an irreducible representation $\mathrm{M}_{d}$ of dimension $d$, which $q$-deforms the $d$-dimensional irreducible representation of $\mathfrak{s l}_{2}$. Tensor products of representations are defined by equipping $\mathcal{U}_{q}\left(\mathfrak{s l}_{2}\right)$ with a Hopf algebra structure, and they decompose into direct sums of irreducible subrepresentations. By trivial representation we mean the one-dimensional representation $\mathrm{M}_{1} \cong \mathbb{C}$ or a direct sum of copies of it. Finally, we say that a vector $v$ in a representation is a highest weight vector if it is annihilated by the raising operator, i.e., $E . v=0$.

To construct $n$-point boundary correlation functions, we form a tensor product of $n$ irreducible representations of the quantum group, and to its vectors we associate certain functions of integral form. We show that under this association, desired properties of the functions follow from natural representation theoretical properties of the vectors. Functions $\mathcal{F}[v]$ associated to highest weight vectors $v$ are well-defined on the chamber domain (1.2), and they satisfy a system of Benoit \& Saint-Aubin partial differential equations. Asymptotics of the functions can be read off from projections to subrepresentations. Homogeneity degree of the function is related to the eigenvalue of the Cartan element $K$, and for vectors in the trivial subrepresentation, the associated function is covariant under all Möbius transformations.

1.3. The main result. We now outline the main result of this article, whose precise statement will be given in Theorems 4.16 and 4.17 in Section 4.8, once all relevant notation and conventions have been introduced. Examples of its applications are discussed in Sections 1.4 and 1.5

For the precise definition of our correspondence, at intermediate steps we need an auxiliary anchor point $x_{0}$, and we have to use functions defined on the restricted chamber

$$
\mathfrak{X}_{n}^{\left(x_{0}\right)}=\left\{\left(x_{1}, \ldots, x_{n}\right) \in \mathbb{R}^{n} \mid x_{0}<x_{1}<\cdots<x_{n}\right\} .
$$

The correspondence consists of linear mappings

$$
\mathcal{F}^{\left(x_{0}\right)}: \bigotimes_{i=1}^{n} \mathrm{M}_{d_{i}} \rightarrow \mathcal{C}^{\infty}\left(\mathfrak{X}_{n}^{\left(x_{0}\right)}\right)
$$

from the tensor product $\bigotimes_{i=1}^{n} \mathrm{M}_{d_{i}}=\mathrm{M}_{d_{n}} \otimes \cdots \otimes \mathrm{M}_{d_{1}}$ of $n$ irreducible representations of the quantum group $\mathcal{U}_{q}\left(\mathfrak{s l}_{2}\right)$ to a space of smooth functions of $n$ variables. By construction, detailed in Section 4.1 . the mapping $\mathcal{F}^{\left(x_{0}\right)}$ sends appropriate basis vectors to functions of the form

$$
C \times \prod_{1 \leq i<j \leq n}\left(x_{j}-x_{i}\right)^{\frac{2}{\kappa}\left(d_{i}-1\right)\left(d_{j}-1\right)} \times \int \cdots \int_{\mathfrak{L} \ni} \prod_{\substack{1 \leq i \leq n \\ 1 \leq r \leq \ell}}\left(w_{r}-x_{i}\right)^{-\frac{4}{\kappa}\left(d_{i}-1\right)} \prod_{1 \leq r<s \leq \ell}\left(w_{s}-w_{r}\right)^{\frac{8}{\kappa}} \mathrm{d} w_{1} \cdots \mathrm{d} w_{\ell},
$$

where $C$ is a phase factor and the integrations are over a family $\mathfrak{L}^{\ni}$ of non-intersecting loops based at the anchor point $x_{0}$. The function depends on the vector through the topology of the integration 


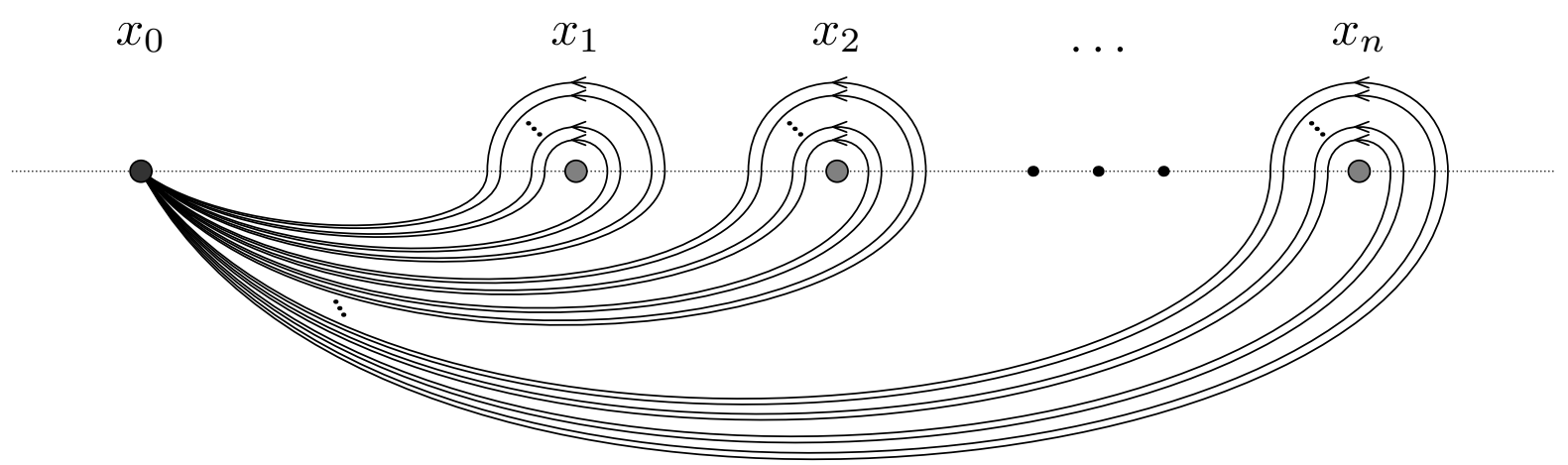

FIGURE 1.1. Under our correspondence, basis vectors in a tensor product representation of a quantum group are mapped to integral form functions. The integration is over a family $\mathfrak{L}^{\ni}$ of non-intersecting loops based at the anchor point $x_{0}$, with nested loops surrounding the points $x_{1}, \ldots, x_{n}$. The numbers of loops and the phase factor $C$ multiplying the integral depend on the basis vector, as detailed in Sections 3.3 and 4.1 .

surface $\mathfrak{L}^{\ni}$, as well as the phase factor $C$. The family $\mathfrak{L}^{\ni}$ of non-intersecting loops is schematically illustrated in Figure 1.1 .

We refer to the mappings $\mathcal{F}^{\left(x_{0}\right)}$ as "the spin chain - Coulomb gas correspondence", since they map the state spaces $\bigotimes_{i=1}^{n} \mathrm{M}_{d_{i}}$ of finite quantum spin chains to spaces of screened correlation functions in the Coulomb gas formalism of conformal field theory. Such integral form correlation functions of CFT have been studied since [DF84].

Our main theorem - informally stated — is the following.

Theorem (Theorems 4.16 and 4.17 in Section 4.8). Under the mappings $v \mapsto \mathcal{F}^{\left(x_{0}\right)}[v]$, properties of the vector $v \in \bigotimes_{i=1}^{n} \mathrm{M}_{d_{i}}$ ensure properties of the corresponding function

$$
\mathcal{F}^{\left(x_{0}\right)}[v]: \mathfrak{X}_{n}^{\left(x_{0}\right)} \rightarrow \mathbb{C}
$$

as follows:

(well-def.): For any highest weight vector $v$, the function $\mathcal{F}^{\left(x_{0}\right)}[v]$ is independent of $x_{0}$, and thus gives rise to a well-defined function on the chamber $\mathfrak{X}_{n}$, denoted by $\mathcal{F}[v]$.

(PDE): If $v$ is a highest weight vector, then the function $F=\mathcal{F}[v]: \mathfrak{X}_{n} \rightarrow \mathbb{C}$ satisfies, for each $j=1, \ldots, n$, the linear homogeneous partial differential equation (1.3) of order $d_{j}$ equal to the dimension of a factor $\mathrm{M}_{d_{j}}$ in the tensor product.

(COV): The functions have the following covariance properties:

- The function $\mathcal{F}[v]$ (resp. $\mathcal{F}^{\left(x_{0}\right)}[v]$ ) is translation invariant.

- If $v$ is an eigenvector of the Cartan generator $K$, then the function $\mathcal{F}[v]$ (resp. $\left.\mathcal{F}^{\left(x_{0}\right)}[v]\right)$ is homogeneous of a degree that depends on the eigenvalue.

- If $v$ belongs to the trivial subrepresentation of $\bigotimes_{i=1}^{n} \mathrm{M}_{d_{i}}$, then $F=\mathcal{F}[v]$ transforms covariantly under all Möbius transformations as in (1.4).

(ASY): Suppose that $v$ belongs to the subrepresentation of $\bigotimes_{i=1}^{n} \mathrm{M}_{d_{i}}$ obtained by picking the $d$ dimensional irreducible direct summand in the tensor product of the $j$ :th and $j+1$ :st factors $\mathbf{M}_{d_{j}}$ and $\mathrm{M}_{d_{j+1}}$, and let

$$
\hat{v} \in\left(\bigotimes_{i>j+1} \mathrm{M}_{d_{i}}\right) \otimes \mathrm{M}_{d} \otimes\left(\bigotimes_{i<j} \mathrm{M}_{d_{i}}\right)
$$


denote the vector obtained by identifying $v$ as a vector in an $(n-1)$-fold tensor product representation. Then, as $\left|x_{j+1}-x_{j}\right| \rightarrow 0$, the function $\mathcal{F}[v]$ has the asymptotic behavior

$$
\mathcal{F}[v] \sim B \times\left(x_{j+1}-x_{j}\right)^{\Delta} \times \mathcal{F}[\hat{v}],
$$

where the constant $B=B_{d}^{d_{j}, d_{j+1}}$ and the exponent $\Delta=\Delta_{d}^{d_{j}, d_{j+1}}$ are explicit. The analogous statement holds also for $\mathcal{F}^{\left(x_{0}\right)}[v]$.

In practice, this theorem is applied as follows. In specific problems, we are looking for particular solutions to systems of PDEs of conformal field theory. Typically, the sought solution has specific Möbius covariance properties and specific boundary conditions as the distance of some of its arguments tend to zero. By our correspondence, the task of finding a function with these properties is translated to the problem of finding a corresponding vector in the tensor product representation. The different parts (PDE), (COV), and (ASY) of the theorem state that a careful choice of the vector would ensure the desired properties of the function - even the most delicate boundary conditions for the function can be guaranteed by (ASY) if the vector has appropriate projections to certain subrepresentations. All of the requirements are explicit linear conditions on the vector living in a finite dimensional vector space, and we moreover have a variety of representation theoretical tools at our disposal to solve for such a vector. By outlining a few case studies in Sections 1.4 and 1.5 , we exemplify how the correspondence thus allows us to translate the original, possibly rather complicated problem to an explicitly solvable one, and to eventually express the function of interest explicitly as a linear combination of integral form functions.

Before example applications, we make a few further observations about the interpretation of the constructed correspondence, and comparisons to related research.

- The functions $\mathcal{F}^{\left(x_{0}\right)}[v]$ are given by integrals over auxiliary screening variables, and slightly informally, the quantum group can be thought of as acting on the integration surfaces: the generator $F$ increases the dimension of the integration surface (i.e., the number of screening variables), and the generator $E$ decreases it.

- A precise version of the quantum group action on integration surfaces has been given by Felder and Wieczerkowski, who define an action of $\mathcal{U}_{q}\left(\mathfrak{s l}_{2}\right)$ on a suitable homology theory [FW91. Our representation of the quantum group could be obtained from this homology via a degenerate evaluation. The evaluation in particular renders the infinite dimensional Verma modules in the work of Felder and Wieczerkowski into just the finite dimensional irreducible representations $\mathrm{M}_{d}$.

- The special properties of the highest weight vectors can be seen to arise from the closedness of the associated integration surface - a version of Stokes' formula can be used in these cases without boundary terms (Lemma 4.7 and Corollary 4.8).

- The most common way to obtain Möbius covariance in the Coulomb gas formalism of conformal field theory is to ensure a charge neutrality, which takes into account a background charge. The integrals associated to vectors in the trivial subrepresentation do not satisfy this simplest charge neutrality, but rather fall short of it precisely by the amount of the background charge. Our full Möbius covariance statement for these vectors requires a little more work (Proposition 4.15).

- In the presentation of this article, we have opted for straightforwardness and self-containedness. We have therefore tried to avoid invoking results from the literature, where they would require significant additional theory - in particular regarding general quantum groups, the homology theory of [FW91, and the structure of charged Fock spaces and properties of vertex operators Fel89, FF90, IK11.

1.4. Two example applications to Schramm-Loewner evolutions. We next illustrate the use of our main result by briefly describing two applications, which both arise from the theory of SLEs.

SLEs are random curves in planar domains that were introduced by Schramm [Sch00] as candidates of scaling limits of interfaces in statistical mechanics models at criticality. SLEs are constructed by a growth process of the curve, encoded in a Loewner chain, in such a way as to ensure the fundamental properties of conformal invariance (of the law associated to different domains) and domain Markov 
property (which describes the conditional law of the continuation of the curve, given a segment of it). In all SLE variants, a single parameter $\kappa>0$ captures some of the most important properties of the curve - in physical terms, $\kappa$ determines the universality class of the underlying statistical mechanics model and the central charge $c(\kappa)$ of the conformal field theory. In our applications, $\kappa$ determines the deformation parameter of the quantum group according to $q=e^{\mathbf{i} \pi 4 / \kappa}$.

The simplest setup of SLEs concerns curves living in a simply connected domain, starting from one marked boundary point and ending at another. A classification result, sometimes referred to as "Schramm's principle", states that such random non-self-traversing curves with domain Markov property and conformally invariant laws are uniquely characterized by the parameter $\kappa$. The term chordal $\mathrm{SLE}_{\kappa}$ is used for these random curves. Chordal $\mathrm{SLE}_{\kappa}$, with particular values of $\kappa$, are known to be the scaling limits of interfaces in the presence of the simplest (Dobrushin) boundary conditions in various critical models of statistical mechanics - see e.g. [Smi01, LSW04, CN07, Zha08, $\left.\mathrm{CDCH}^{+} 14\right]$.

1.4.1. Application to multiple SLEs. Multiple SLEs arise from trying to generalize Schramm's principle to cases where several interfaces are present: in a simply connected domain with $2 N$ marked boundary points we may have $N$ curves connecting pairwise the marked points. Such processes have been studied in Dub07, BBK05, Gra07, KL07, PW17, and in some cases they are known to be the scaling limits of lattice model interfaces in the presence of alternating boundary conditions [zy11, CS12, KS18].

Multiple random curves with conformally invariant laws and domain Markov properties are no longer specified by the parameter $\kappa$ alone, but for a fixed $\kappa<8$, a finite dimensional convex set of possible laws exists. The extremal points of the set of multiple SLE laws were called pure geometries in [BBK05]. Coulomb gas integrals for this problem were considered in Dub06, Kyt07. The problem of explicit description of the pure geometries is very closely related to crossing probabilities, for which formulas using Coulomb gas integrals have been recently obtained in the series of articles FK15a, FK15b, FK15c, FK15d, FSKZ17]. In comparison with the approaches of [Dub06, FK15a, the Coulomb gas integrals in our approach have the advantage of treating all the $2 N$ marked points on equal footing, at the expense of increasing the dimension of the integration surface by one.

As summarized precisely in [KP16, Appendix A], an explicit Loewner chain construction of a multiple SLE uses a partition function Dub07, BBK05, which is most convenient to write down for the reference domain of the upper half-plane $\mathbb{H}=\{z \in \mathbb{C} \mid \Im \mathfrak{m}(z)>0\}$ as

$$
\mathcal{Z}\left(x_{1}, \ldots, x_{2 N}\right)
$$

where the marked points on the real line $x_{i} \in \mathbb{R}=\partial \mathbb{H}$, for $i=1, \ldots, 2 N$, are ordered as

$$
x_{1}<\cdots<x_{2 N} .
$$

A stochastic reparametrization invariance of the random curves requires the PDEs

$$
\left[\frac{\kappa}{2} \frac{\partial^{2}}{\partial x_{i}^{2}}+\sum_{j \neq i}\left(\frac{2}{x_{j}-x_{i}} \frac{\partial}{\partial x_{j}}-\frac{2 h}{\left(x_{j}-x_{i}\right)^{2}}\right)\right] \mathcal{Z}\left(x_{1}, \ldots, x_{2 N}\right)=0 \quad \text { for all } i=1, \ldots, 2 N,
$$

where $h=\frac{6-\kappa}{2 \kappa}$, see [Dub07]. Moreover, conformal invariance of the law of the random curves requires the following Möbius covariance:

$$
\mathcal{Z}\left(x_{1}, \ldots, x_{2 N}\right)=\prod_{i=1}^{2 N} \mu^{\prime}\left(x_{i}\right)^{h} \times \mathcal{Z}\left(\mu\left(x_{1}\right), \ldots, \mu\left(x_{2 N}\right)\right)
$$

for any conformal map $\mu$ of $\mathbb{H}$ onto itself, which preserves the order of the marked points.

The pure geometries of multiple SLEs are labeled by planar pair partitions $\alpha$ of $2 N$ points, and the problem is to find the corresponding partition functions $\mathcal{Z}_{\alpha}$. The requirements of covariance (1.7) and PDEs (1.6) are the same for all pure geometries $\alpha$, but the boundary conditions depend on $\alpha$. The 
asymptotic behavior of $\mathcal{Z}_{\alpha}$ as $\left|x_{j+1}-x_{j}\right| \rightarrow 0$ depends on whether the points indexed $j$ and $j+1$ form a pair of $\alpha$ or not - see [BBK05, KP16, PW17]. More precisely, when $x_{j-1}<\xi<x_{j+2}$, we have

$$
\lim _{x_{j}, x_{j+1} \rightarrow \xi} \frac{\mathcal{Z}_{\alpha}\left(x_{1}, \ldots, x_{2 N}\right)}{\left|x_{j+1}-x_{j}\right|^{\Delta}}=\left\{\begin{array}{ll}
0 & \text { if }\{j, j+1\} \notin \alpha \\
\mathcal{Z}_{\hat{\alpha}}\left(x_{1}, \ldots, x_{j-1}, x_{j+2}, \ldots, x_{2 N}\right) & \text { if }\{j, j+1\} \in \alpha
\end{array},\right.
$$

where $\Delta=-2 h=\frac{\kappa-6}{\kappa}$, and $\hat{\alpha}$ is the planar pair partition of $2 N-2$ points obtained from $\alpha$ by removing the pair $\{j, j+1\}$.

The results of the present article are used in [KP16 to explicitly construct solutions to 1.6, 1.7 , and $(1.8)$, in the form $\mathcal{Z}_{\alpha}=\mathcal{F}\left[v_{\alpha}\right]$, where $\mathcal{F}$ is our spin chain - Coulomb gas correspondence map. Specifically, one forms the $2 N$-fold tensor product of two-dimensional irreducibles $\mathrm{M}_{2}$ of the quantum group. The trivial subrepresentation in this tensor product, consisting of vectors $v \in \mathrm{M}_{2}^{\otimes 2 N}$ such that $E . v=0$ and $K . v=v$, is of dimension equal to the Catalan number $\mathrm{C}_{N}=\frac{1}{N+1}\left(\begin{array}{c}2 N \\ N\end{array}\right)$, which coincides with the number of planar pair partitions of $2 N$ points. One then wants to judiciously choose in this subrepresentation $\mathrm{C}_{N}$ linearly independent vectors

$$
v_{\alpha} \in \mathrm{M}_{2}^{\otimes 2 N},
$$

indexed by the the planar pair partitions $\alpha$, so that $\mathcal{Z}_{\alpha}=\mathcal{F}\left[v_{\alpha}\right]$ will be the desired multiple SLE partition functions of the pure geometry $\alpha$.

For vectors $v_{\alpha} \in \mathrm{M}_{2}^{\otimes 2 N}$, the $2 N$ differential equations of order two, guaranteed by the (PDE) part of our main theorem, turn out to be exactly the equations (1.6) needed for the reparametrization invariance of the multiple SLE. Moreover, the full Möbius covariance guaranteed by the (COV) part is exactly (1.7), as we wanted. The main task is then to choose $v_{\alpha}$ in such a way that the boundary conditions (1.8) are satisfied. For this, the (ASY) part will be used.

The asymptotics property (ASY) refers to the decomposition of a tensor product of two representations into irreducible subrepresentations, which in this case simply reads

$$
M_{2} \otimes M_{2} \cong M_{1} \oplus M_{3} \text {. }
$$

For this case, the possible exponent values appearing in the statement (ASY) are $\Delta_{1}^{2,2}=-2 h=\frac{\kappa-6}{\kappa}$ and $\Delta_{3}^{2,2}=\frac{2}{\kappa}>\Delta_{1}^{2,2}$. Letting $\hat{\pi}_{j, j+1}^{(1)}$ denote the projection determined by picking the one-dimensional irreducible in the direct sum decomposition of the tensor product of the $j$ :th and $j+1$ :st factors, Equation 1.8 will be guaranteed if we have

$$
\hat{\pi}_{j, j+1}^{(1)}\left(v_{\alpha}\right)=\left\{\begin{array}{ll}
0 & \text { if }\{j, j+1\} \notin \alpha \\
\frac{1}{B} v_{\hat{\alpha}} & \text { if }\{j, j+1\} \in \alpha
\end{array},\right.
$$

where $v_{\hat{\alpha}} \in \mathrm{M}_{2}^{\otimes(2 N-2)}$ is the vector corresponding to the planar pair partition $\hat{\alpha}$ of $2 N-2$ points obtained from $\alpha$ by removing the pair $\{j, j+1\}$.

In [KP16], this problem is analyzed in detail, and in particular, it is shown that there is a unique collection of vectors $v_{\alpha}$ satisfying the above requirements, up to an overall normalization. The explicit construction of the multiple SLE partition functions $\mathcal{Z}_{\alpha}=\mathcal{F}\left[v_{\alpha}\right]$ for the pure geometries thus crucially relies on the results of the present article.

1.4.2. Application to boundary visit probabilities for chordal SLE. For concrete probabilistic information about the SLE random curves, it is natural to study the probabilities for SLE curves to visit small neighborhoods of given points. In fact, probabilities to visit infinitesimal neighborhoods can be appropriately renormalized to obtain finite amplitudes known as SLE Green's functions, see [LS11, AKL12, LW13, LZ13, Law15]. A second application of our method, considered in [JJK16], concerns finding explicit formulas for the order-refined multi-point boundary Green's function of the chordal SLE, i.e., the probability amplitude for visits to several boundary points in a prescribed order. 
For simplicity, we fix the domain to be the upper half-plane $\mathbb{H}=\{z \in \mathbb{C} \mid \Im \mathfrak{m}(z)>0\}$, the starting point at $x \in \mathbb{R}$ and the end point at $\infty$. We order the points to be visited on the real line $\mathbb{R}=\partial \mathbb{H}$, and label them by a superscript - or + , according to whether they are on the left or right of the starting point $x$, and thus denote

$$
y_{L}^{-}<\cdots<y_{2}^{-}<y_{1}^{-}<x<y_{1}^{+}<y_{2}^{+}<\cdots<y_{R}^{+},
$$

where $L$ and $R$ are the number of points to be visited on the left and right, respectively. With a given order $\omega$ of visits, a suitably renormalized probability of visits

$$
\zeta_{\omega}\left(y_{L}^{-}, \ldots, y_{1}^{-}, x, y_{1}^{+}, \ldots, y_{R}^{+}\right)
$$

can then be defined, see [JJK16 for details. The task is to find an explicit expression for it. This function $\zeta_{\omega}$ is by construction translation invariant and homogeneous. It can moreover be argued to satisfy linear homogeneous partial differential equations: a second order equation, and $L+R$ third order equations. These PDEs are of the Benoit \& Saint-Aubin type (1.3). They do not depend on the order $\omega$ of visits, but again the boundary conditions do. When two of the arguments are close to each other, the probabilities of visits are asymptotic to similar ones with one point removed from the list of visits. Here we content ourselves to noting that these conditions amount to specifying the asymptotic behavior of $\zeta_{\omega}$ on a codimension one boundary of its domain of definition: they concern the cases when either $\left|y_{i+1}^{ \pm}-y_{i}^{ \pm}\right| \rightarrow 0$ or $\left|y_{1}^{ \pm}-x\right| \rightarrow 0$. For the full list of equations and boundary conditions for this chordal SLE boundary visit question, we refer to [JJK16.

To apply the correspondence of the present article to the problem of finding these boundary visit probabilities of chordal SLE, one considers the tensor product representation

$$
\mathrm{M}_{3}^{\otimes R} \otimes \mathrm{M}_{2} \otimes \mathrm{M}_{3}^{\otimes L}
$$

Again the task is to judiciously choose vectors $v_{\omega}$ in it, such that the multi-point boundary Green's function corresponding to the visit order $\omega$ can be obtained in the form $\zeta_{\omega}=\mathcal{F}\left[v_{\omega}\right]$. The highest weight vector condition E. $v_{\omega}=0$ guarantees, by the (PDE) part of our main theorem, the desired second and third order partial differential equations, and the Cartan generator eigenvalue equation $K \cdot v_{\omega}=q v_{\omega}$ guarantees, by the (COV) part, the correct homogeneity degree in addition to translation invariance. The most subtle requirement is the boundary conditions when either $\left|y_{i+1}^{ \pm}-y_{i}^{ \pm}\right| \rightarrow 0$ or $\left|y_{1}^{ \pm}-x\right| \rightarrow 0$. The (ASY) part is again suitable for this purpose: the decomposition $M_{3} \otimes M_{3} \cong M_{1} \oplus M_{3} \oplus M_{5}$ applies to the case $\left|y_{i+1}^{ \pm}-y_{i}^{ \pm}\right| \rightarrow 0$, and the decompositions $\mathrm{M}_{3} \otimes \mathrm{M}_{2} \cong \mathrm{M}_{2} \oplus \mathrm{M}_{4}$ and $\mathrm{M}_{2} \otimes \mathrm{M}_{3} \cong \mathrm{M}_{2} \oplus \mathrm{M}_{4}$ apply respectively to the cases $\left|y_{1}^{+}-x\right| \rightarrow 0$ and $\left|x-y_{1}^{-}\right| \rightarrow 0$. SLE boundary visits are treated in more detail in [JJK16], and the requirements for the vectors $v_{\omega}$ are shown to have a unique solution in general in [KP16, Section 5]. Again, the main results of the present article are thus instrumental for finding the explicit formulas for these order-refined multi-point boundary visit probabilities of the chordal SLE.

1.5. Application to monodromy invariant bulk correlation functions. The first applications of the spin chain - Coulomb gas correspondence described above concern boundary correlation functions relevant for random conformally invariant curves. We next briefly describe an application to bulk correlation functions of conformal field theory, presented in detail in [FP18b].

In statistical mechanics models at criticality, the scaling limits of both boundary and bulk correlation functions are argued to be described by conformal field theory, and in particular, to be Möbius covariant and satisfy partial differential equations such as the ones studied in this article. The solutions of the partial differential equations are in general multivalued, whereas bulk correlation functions in statistical mechanics models are usually manifestly single-valued. In [FP18b], Flores and Peltola show how to use the spin chain - Coulomb gas correspondence of the present article to construct such bulk correlation functions of conformal field theory. The key idea is again to translate the task of finding the appropriate functions to the task of finding vectors with corresponding properties in a finite-dimensional representation of the quantum group. 
In the construction of [FP18b], the bulk correlation function of $n$ spinless primary fields of conformal dimensions $2 h_{1, d_{j}}$, with $j=1, \ldots, n$, is associated to a vector in the tensor product representation $\left(\bigotimes_{j=1}^{n} \mathrm{M}_{d_{j}}\right)^{\otimes 2}$. The function associated to a simple tensor $v \otimes \bar{v}$, with $v, \bar{v} \in \bigotimes_{j=1}^{n} \mathrm{M}_{d_{j}}$, is

$$
\mathcal{F}^{\left(x_{0}\right)}[v]\left(z_{1}, \ldots, z_{n}\right) \times \mathcal{F}^{\left(x_{0}\right)}[\bar{v}]\left(\bar{z}_{1}, \ldots, \bar{z}_{n}\right),
$$

where the two factors are the analytic continuations of the functions $\mathcal{F}^{\left(x_{0}\right)}[v], \mathcal{F}^{\left(x_{0}\right)}[\bar{v}]: \mathfrak{X}_{n}^{\left(x_{0}\right)} \rightarrow \mathbb{C}$ constructed and studied in the present article. For a function of the form $(1.9)$, the partial differential equations and covariance required of bulk correlation functions are guaranteed by (PDE) and (COV) parts of our main theorem, respectively, when the components $v$ and $\bar{v}$ lie in the trivial subrepresentation of $\bigotimes_{j=1}^{n} \mathrm{M}_{d_{j}}$.

In general, however, the function $(1.9)$ is multivalued, and its monodromy around a loop that winds $z_{j}$ positively around $z_{j+1}$ is given by the action of the R-matrix of the quantum group on the $j$ :th and $j+1$ :st components of $v$ and a conjugate R-matrix on the $j$ :th and $j+1$ :st components of $\bar{v}$. Arbitrary monodromies are described by the representation of the pure braid group on $n$ strands generated by such loops. The function is single-valued precisely when the corresponding vector is invariant under the pure braid group. Using a generalization of a quantum Schur-Weyl duality [FP18a], Flores and Peltola show the uniqueness (up to scalar multiples) of a vector $\sum_{k} v_{k} \otimes \bar{v}_{k}$ which is both braiding invariant and for which $v_{k}, \bar{v}_{k}$ lie in the trivial subrepresentation. This gives the uniqueness of and an explicit expression for the single-valued bulk correlation function.

The spin chain - Coulomb gas correspondence developed in the present article thus also underlies the construction and analysis in [FP18b] of the monodromy invariant bulk correlation function of conformal field theory.

1.6. Organization of this article. In Section 2, we introduce notation, fix conventions, and prove auxiliary results about $q$-combinatorics, the quantum group $\mathcal{U}_{q}\left(\mathfrak{s l}_{2}\right)$, its representations, and their tensor products. Section 3 contains definitions and properties of functions that are used in defining and studying the correspondence. This part does not use the quantum group in any way - only some $q$-combinatorial lemmas are used. Section 4 begins with the definition of the correspondence, and proceeds with the proofs of the properties stated in the main theorems in the order that we have found the most straightforward. It concludes with the precise statement of the main result, Theorems 4.16 and 4.17. In Section 5, we treat two further properties: a generalization of the asymptotics statement and a hidden manifestation of the periodicity of the domain boundary. From the main text, we postpone some necessary but routine contour manipulation arguments to Appendix A, and the lengthy proof of a lemma about differential operators to Appendix B Finally, in Appendix C, we give formulations of our main results with alternative conventions, which may occasionally appear more natural.

Acknowledgments. We thank Michel Bauer, Denis Bernard, Dmitry Chelkak, Steven Flores, Philippe Di Francesco, Azat Gainutdinov, Christian Hagendorf, Clément Hongler, Konstantin Izyurov, Niko Jokela, Matti Järvinen, Peter Kleban, Hubert Saleur, and Jacob Simmons for interesting discussions, useful comments, and suggested improvements. We also thank the anonymous referees for useful suggestions.

This work was supported by the Academy of Finland. E.P. was supported by the Finnish National Doctoral Programme in Mathematics and its Applications and Vilho, Yrjö and Kalle Väisälä Foundation. The work was carried out while E.P. was affiliated with the University of Helsinki.

\section{The QUANTUM GROUP}

The main purpose of this section is to fix notation and conventions about the quantum group $\mathcal{U}_{q}\left(\mathfrak{s l}_{2}\right)$. We also include auxiliary results of $q$-combinatorial flavor, which are needed later on in the article. 
2.1. Q-numbers and some combinatorial formulas. Let $q \in \mathbb{C} \backslash\{0\}$, and assume further that $q$ is not a root of unity, i.e. $q^{m} \neq 1$ for all $m \in \mathbb{Z} \backslash\{0\}$. Define, for $m \in \mathbb{Z}$ and for $n, k \in \mathbb{N}, 0 \leq k \leq n$, the $q$-integers as

$$
\begin{aligned}
{[m] } & =\frac{q^{m}-q^{-m}}{q-q^{-1}} \\
& =q^{m-1}+q^{m-3}+\cdots+q^{3-m}+q^{1-m},
\end{aligned}
$$

the $q$-factorials as

$$
[n] !=\prod_{m=1}^{n}[m]
$$

and the $q$-binomial coefficients as

$$
\left[\begin{array}{l}
n \\
k
\end{array}\right]=\frac{[n] !}{[k] ![n-k] !} .
$$

The following " $q$-combinatorial formulas" will be used in later calculations: specifically, part (a) will be used in Lemma 4.7, part (b) in Lemma 3.2 part (c) in Lemma 3.6. and part (d) in Lemma 4.1

\section{Lemma 2.1.}

(a): We have

$$
[\ell][d-\ell]=\frac{1}{q-q^{-1}} \sum_{u=0}^{\ell-1}\left(q^{d-1-2 u}-q^{-d+1+2 u}\right) .
$$

(b): For a permutation $\sigma \in \mathfrak{S}_{n}$ of $\{1, \ldots, n\}$, denote the set of inversions of $\sigma$ by

$$
\operatorname{inv}(\sigma)=\{(i, j) \mid i<j \text { and } \sigma(i)>\sigma(j)\} \text {. }
$$

Then we have

$$
\sum_{\sigma \in \mathfrak{S}_{n}} q^{-2 \times \# \operatorname{inv}(\sigma)}=q^{-\left(\begin{array}{c}
n \\
2
\end{array}\right)}[n] !
$$

(c): We have

$$
\sum_{1 \leq r_{1}<r_{2}<\cdots<r_{k} \leq n} q^{-2 \sum_{j=1}^{k}\left(r_{j}-j\right)}=q^{-k(n-k)}\left[\begin{array}{l}
n \\
k
\end{array}\right] .
$$

(d): We have, for any $\beta$ and $n \in \mathbb{N}$

$$
\sum_{m=0}^{n}\left[\begin{array}{c}
n \\
m
\end{array}\right](-1)^{m} q^{m \beta}=q^{\frac{1}{2} n \beta} \prod_{s=0}^{n-1}\left(q^{\frac{1}{2}(n-1-\beta)-s}-q^{\frac{1}{2}(\beta+1-n)+s}\right) .
$$

Proof. For part (a), use the definition $[d-\ell]=\frac{q^{d-\ell}-q^{\ell-d}}{q-q^{-1}}$ and the finite geometric series $[\ell]=q^{\ell-1}+$ $q^{\ell-3}+\cdots+q^{-\ell+1}$ to get the asserted formula

$$
\begin{aligned}
\left(q-q^{-1}\right)[\ell][d-\ell] & =\left(q^{d-\ell}-q^{\ell-d}\right)\left(q^{\ell-1}+q^{\ell-3}+\cdots+q^{-\ell+1}\right) \\
& =\left(q^{d-1}+q^{d-3}+\cdots+q^{d-2 \ell+1}\right)-\left(q^{2 \ell-1-d}+q^{2 \ell-3-d}+\cdots+q^{-d+1}\right) .
\end{aligned}
$$

For part (b), use the same finite geometric series to rewrite the right hand side as

$$
q^{-\left(\begin{array}{c}
n \\
2
\end{array}\right)}[n] !=\prod_{k=1}^{n}\left(\sum_{l=0}^{k-1} q^{-2 l}\right) .
$$


The inversions $(i, j) \in \operatorname{inv}(\sigma)$ of $\sigma \in \mathfrak{S}_{n}$ can be grouped according to the smaller index $i$ : we have

$$
\begin{aligned}
& \# \operatorname{inv}(\sigma)=\sum_{i=1}^{n} \# \operatorname{inv}_{i}(\sigma), \quad \text { where } \\
& \operatorname{inv}_{i}(\sigma)=\{j \mid j>i \text { and } \sigma(j)<\sigma(i)\} .
\end{aligned}
$$

In fact, permutations $\sigma$ are in bijection with the sequences $\left(\# \operatorname{inv}_{i}(\sigma)\right)_{i=1}^{n}$. Then, in the expansion of the product $\prod_{k=1}^{n}\left(\sum_{l=0}^{k-1} q^{-2 l}\right)$ the choice of the term in the $k$ :th factor can be thought of as corresponding to the choice of \#inv ${ }_{n+1-k}(\sigma)$. The product is thus expanded as a sum over permutations $\sigma$, with coefficients $\prod_{i=1}^{n} q^{-2 \# \operatorname{inv}_{i}(\sigma)}=q^{-2 \# \operatorname{inv}(\sigma)}$.

The proofs of (c) and (d) are both based on $q$-Pascal triangles. Let $L_{k}^{(n)}$ and $R_{k}^{(n)}$ denote the left hand side and right hand side of assertion (c), respectively. Splitting the sum defining $L_{k}^{(n)}$ according to whether $r_{k}=n$ or $r_{k}<n$, we obtain a Pascal triangle type recursion

$$
L_{k}^{(n)}=q^{-2(n-k)} L_{k-1}^{(n-1)}+L_{k}^{(n-1)} .
$$

A straightforward calculation, using the definition (2.1) of $q$-integers, shows that we also have the recursion $R_{k}^{(n)}=q^{-2(n-k)} R_{k-1}^{(n-1)}+R_{k}^{(n-1)}$. The equality $L_{k}^{(n)}=R_{k}^{(n)}$ follows from this recursion, together with the initial observation $L_{k}^{(1)}=R_{k}^{(1)}$.

For the proof of (d), proceed by induction on $n$. For $n=0$ both sides are equal to 1 . Let $R^{(n)}(\beta)$ denote the right hand side, and note that we can write

$$
R^{(n+1)}(\beta)=\left(1-q^{n+\beta}\right) \times R^{(n)}(\beta-1) .
$$

Assuming the asserted formula for $R^{(n)}(\beta-1)$, we expand in powers of $q^{\beta}$ as follows

$$
R^{(n+1)}(\beta)=\sum_{m}(-1)^{m} q^{m \beta}\left(q^{-m}\left[\begin{array}{c}
n \\
m
\end{array}\right]+q^{1+n-m}\left[\begin{array}{c}
n \\
m-1
\end{array}\right]\right) .
$$

It now suffices to apply the following simple identity

$$
q^{-m}\left[\begin{array}{c}
n \\
m
\end{array}\right]+q^{1+n-m}\left[\begin{array}{c}
n \\
m-1
\end{array}\right]=\left[\begin{array}{c}
n+1 \\
m
\end{array}\right] .
$$

2.2. The quantum group and its representations. We now give a definition of the quantum group $\mathcal{U}_{q}\left(\mathfrak{s l}_{2}\right)$ by generators and relations. We also concretely describe the irreducible representations $\mathrm{M}_{d}$, and record needed results about the decompositions of their tensor products.

2.2.1. Definition of the quantum group. The quantum group $\mathcal{U}_{q}\left(\mathfrak{s l}_{2}\right)$ is the associative unital algebra over $\mathbb{C}$ generated by $E, F, K, K^{-1}$ subject to the relations

$$
\begin{aligned}
K K^{-1} & =1=K^{-1} K, \quad K E=q^{2} E K, \quad K F=q^{-2} F K, \\
E F-F E & =\frac{1}{q-q^{-1}}\left(K-K^{-1}\right) .
\end{aligned}
$$

There is a unique Hopf algebra structure on $\mathcal{U}_{q}\left(\mathfrak{s l}_{2}\right)$ with the coproduct, an algebra homomorphism

$$
\Delta: \mathcal{U}_{q}\left(\mathfrak{s l}_{2}\right) \rightarrow \mathcal{U}_{q}\left(\mathfrak{s l}_{2}\right) \otimes \mathcal{U}_{q}\left(\mathfrak{s l}_{2}\right),
$$

given on the generators by the expressions

$$
\Delta(E)=E \otimes K+1 \otimes E, \quad \Delta(K)=K \otimes K, \quad \Delta(F)=F \otimes 1+K^{-1} \otimes F .
$$


With the coproduct, we make the tensor product of two representations $M^{\prime}$ and $M^{\prime \prime}$ again a representation. The action of $\mathcal{U}_{q}\left(\mathfrak{s l}_{2}\right)$ on $M^{\prime} \otimes M^{\prime \prime}$ is defined so that if

$$
\Delta(X)=\sum_{i} X_{i}^{\prime} \otimes X_{i}^{\prime \prime} \in \mathcal{U}_{q}\left(\mathfrak{s l}_{2}\right) \otimes \mathcal{U}_{q}\left(\mathfrak{s l}_{2}\right)
$$

and $v^{\prime} \in M^{\prime}, v^{\prime \prime} \in M^{\prime \prime}$, then

$$
X .\left(v^{\prime} \otimes v^{\prime \prime}\right)=\sum_{i}\left(X_{i}^{\prime} \cdot v^{\prime}\right) \otimes\left(X_{i}^{\prime \prime} \cdot v^{\prime \prime}\right) \in M^{\prime} \otimes M^{\prime \prime} .
$$

Note that we generally cannot canonically identify $M^{\prime} \otimes M^{\prime \prime}$ with $M^{\prime \prime} \otimes M^{\prime}$ as representations, because the coproduct $\Delta$ is not cocommutative. However, the coproduct is coassociative, that is (id $\otimes \Delta) \circ \Delta=$ $(\Delta \otimes \mathrm{id}) \circ \Delta$, and therefore the canonical identification $\left(M^{\prime} \otimes M^{\prime \prime}\right) \otimes M^{\prime \prime \prime} \cong M^{\prime} \otimes\left(M^{\prime \prime} \otimes M^{\prime \prime \prime}\right)$ is an isomorphism of representations. More generally, we may talk about multiple tensor products without specifying the positions of parentheses. For calculations with $n$-fold tensor products, one needs the $(n-1)$-fold coproduct $\Delta^{(n)}: \mathcal{U}_{q}\left(\mathfrak{s l}_{2}\right) \rightarrow\left(\mathcal{U}_{q}\left(\mathfrak{s l}_{2}\right)\right)^{\otimes n}$

$$
\Delta^{(n)}=\left(\Delta \otimes \mathrm{id}^{\otimes(n-2)}\right) \circ\left(\Delta \otimes \mathrm{id}^{\otimes(n-3)}\right) \circ \cdots \circ(\Delta \otimes \mathrm{id}) \circ \Delta .
$$

We record the following easily verified expressions for the $(n-1)$-fold coproducts of the generators for later use in e.g. Lemmas 4.3 and 5.3 . Propositions 4.15 and 5.1 and Corollary 4.8 .

Lemma 2.2. We have

$$
\begin{aligned}
& \Delta^{(n)}(K)=K^{\otimes n} \\
& \Delta^{(n)}(E)=\sum_{i=1}^{n} 1^{\otimes(n-i)} \otimes E \otimes K^{\otimes(i-1)} \\
& \Delta^{(n)}(F)=\sum_{i=1}^{n}\left(K^{-1}\right)^{\otimes(n-i)} \otimes F \otimes 1^{\otimes(i-1)} .
\end{aligned}
$$

2.2.2. Irreducible representations of the quantum group. We will use representations which can be thought of as $q$-deformations of the irreducible representations of the semisimple Lie algebra $\mathfrak{s l}_{2}$. For the statement of the lemma below, recall the definition of $q$-integers $[m]$ from Equation (2.1).

Lemma 2.3. For every positive integer $d$, there is an irreducible representation $\mathrm{M}_{d}$ of $\mathcal{U}_{q}\left(\mathfrak{s i}_{2}\right)$ with a basis $e_{0}^{(d)}, e_{1}^{(d)}, \ldots, e_{d-1}^{(d)}$ and the action of the generators defined by

$$
\begin{aligned}
& K . e_{l}^{(d)}=q^{d-1-2 l} e_{l}^{(d)} \\
& F . e_{l}^{(d)}= \begin{cases}e_{l+1}^{(d)} & \text { if } l \neq d-1 \\
0 & \text { if } l=d-1\end{cases} \\
& E . e_{l}^{(d)}=\left\{\begin{array}{ll}
{[l][d-l] e_{l-1}^{(d)}} & \text { if } l \neq 0 \\
0 & \text { if } l=0
\end{array} .\right.
\end{aligned}
$$

Any d-dimensional irreducible representation of $\mathcal{U}_{q}\left(\mathfrak{s l}_{2}\right)$, where the $K$-eigenvalues are integer powers of $q$, is isomorphic to $\mathrm{M}_{d}$.

Proof. It is easy to check that the formulas defining the action respect the relations 2.5). Moreover, since the $q$-integers $[m]$ are non-vanishing for $m \neq 0, \mathbf{M}_{d}$ is clearly irreducible. If $V$ is an irreducible representation, then $K$ is diagonalizable on $V$, because the sum of $K$-eigenspaces is a subrepresentation. If in a finite dimensional irreducible representation $V$ the $K$-eigenvalues are integer powers of $q$, then an eigenvector $v_{0}$ of $K$ of eigenvalue $q^{m_{0}}$ with $m_{0} \in \mathbb{Z}$ maximal must be annihilated by $E$. By a standard calculation one then shows that the linear span of the $m_{0}+1$ vectors $v_{0}, F . v_{0}, F^{2} . v_{0}, \ldots, F^{m_{0}} . v_{0}$ forms a subrepresentation isomorphic to $\mathrm{M}_{m_{0}+1}$. The last assertion follows from this. 
2.2.3. Tensor products of the irreducible representations. Tensor products of the representations defined in Section 2.2.2 decompose to direct sums of irreducible subrepresentations of the same type. Concrete descriptions of such decompositions, as given in the following $q$-analogue of the Clebsch-Gordan formulas, will be needed especially in Lemmas $4.1-4.3$ Propositions $4.4-4.5$ and in Section 5.3 . Recall that the action of $\mathcal{U}_{q}\left(\mathfrak{s l}_{2}\right)$ on tensor products is defined using the coproduct 2.6), and recall also the definition of $q$-factorials $[n]$ ! from Equation (2.3).

Lemma 2.4. Consider the tensor product representation $\mathrm{M}_{d^{\prime \prime}} \otimes \mathrm{M}_{d^{\prime}}$. For any

$$
m \in\left\{0,1, \ldots, \min \left(d^{\prime}, d^{\prime \prime}\right)-1\right\},
$$

denote $d=d^{\prime}+d^{\prime \prime}-1-2 m$, and define

$$
T_{0 ; m}^{l^{\prime}, l^{\prime \prime}}=T_{0 ; m}^{l^{\prime}, l^{\prime \prime}}\left(d^{\prime}, d^{\prime \prime}\right)=\delta_{l^{\prime}+l^{\prime \prime}, m} \times(-1)^{l^{\prime}} \frac{\left[d^{\prime}-1-l^{\prime}\right] !\left[d^{\prime \prime}-1-l^{\prime \prime}\right] !}{\left[l^{\prime}\right] !\left[d^{\prime}-1\right] !\left[l^{\prime \prime}\right] !\left[d^{\prime \prime}-1\right] !} \frac{q^{l^{\prime}\left(d^{\prime}-l^{\prime}\right)}}{\left(q-q^{-1}\right)^{m}}
$$

Then the vector

$$
\tau_{0}=\tau_{0}^{\left(d ; d^{\prime}, d^{\prime \prime}\right)}=\sum_{l^{\prime}, l^{\prime \prime}} T_{0 ; m}^{l^{\prime}, l^{\prime \prime}} \times\left(e_{l^{\prime \prime}} \otimes e_{l^{\prime}}\right)
$$

satisfies E. $\tau_{0}=0$ and $K . \tau_{0}=q^{d-1} \tau_{0}$ (i.e., $\tau_{0}$ is a highest weight vector). The subrepresentation of $\mathrm{M}_{d^{\prime \prime}} \otimes \mathrm{M}_{d^{\prime}}$ generated by $\tau_{0}$ is isomorphic to $\mathrm{M}_{d}$.

The tensor product representation decomposes to a direct sum of irreducibles

$$
\mathrm{M}_{d^{\prime \prime}} \otimes \mathrm{M}_{d^{\prime}} \cong \mathrm{M}_{d^{\prime}+d^{\prime \prime}-1} \oplus \mathrm{M}_{d^{\prime}+d^{\prime \prime}-3} \oplus \cdots \oplus \mathrm{M}_{\left|d^{\prime}-d^{\prime \prime}\right|+3} \oplus \mathrm{M}_{\left|d^{\prime}-d^{\prime \prime}\right|+1} .
$$

Proof. Because the coefficients $T_{0 ; m}^{l^{\prime}, l^{\prime \prime}}$ above are non-zero only for $l^{\prime}+l^{\prime \prime}=m$, we can write the vector $\tau_{0}$ as $\tau_{0}=\sum_{k} T_{0 ; m}^{k, m-k} \times\left(e_{m-k} \otimes e_{k}\right)$. Since $\Delta(K)=K \otimes K$, we have

$$
K .\left(e_{m-k} \otimes e_{k}\right)=\left(q^{d^{\prime \prime}-1-2(m-k)} e_{m-k}\right) \otimes\left(q^{d^{\prime}-1-2 k} e_{k}\right)=q^{d-1}\left(e_{m-k} \otimes e_{k}\right)
$$

with $d=d^{\prime}+d^{\prime \prime}-1-2 m$, and therefore obviously $K . \tau_{0}=q^{d-1} \tau_{0}$. Using $\Delta(E)=E \otimes K+1 \otimes E$, we get

$$
E .\left(e_{m-k} \otimes e_{k}\right)=[m-k]\left[d^{\prime \prime}-m+k\right] q^{d^{\prime}-1-2 k}\left(e_{m-k-1} \otimes e_{k}\right)+[k]\left[d^{\prime}-k\right]\left(e_{m-k} \otimes e_{k-1}\right),
$$

and thus

$$
\begin{aligned}
E . \tau_{0}=\sum_{k}([m-k] & {\left[d^{\prime \prime}-m+k\right] q^{d^{\prime}-1-2 k} T_{0 ; m}^{k, m-k} } \\
& \left.+[k+1]\left[d^{\prime}-k-1\right] T_{0 ; m}^{k+1, m-1-k}\right) \times\left(e_{m-k-1} \otimes e_{k}\right) .
\end{aligned}
$$

The coefficients satisfy $T_{0 ; m}^{k+1, m-1-k}=-T_{0 ; m}^{k, m-k} \times \frac{[m-k]\left[d^{\prime \prime}-m+k\right]}{[k+1]\left[d^{\prime}-k-1\right]} q^{d^{\prime}-1-2 k}$, and hence, we get E. $\tau_{0}=0$.

From Lemma 2.3 and the properties E. $\tau_{0}=0$ and $K . \tau_{0}=q^{d-1} \tau_{0}$ it is clear that the vector $\tau_{0}$ generates a subrepresentation of $\mathrm{M}_{d^{\prime \prime}} \otimes \mathrm{M}_{d^{\prime}}$ isomorphic to $\mathrm{M}_{d}$. The dimension $d^{\prime} d^{\prime \prime}$ of the representation $\mathrm{M}_{d^{\prime \prime}} \otimes \mathrm{M}_{d^{\prime}}$ equals the sum of dimensions $d=d^{\prime}+d^{\prime \prime}-1-2 m$ over the allowed values of $m$. Thus, the entire representation is a direct sum of these subrepresentations.

Remark 2.5. In view of Equation 2.9), we may freely interpret $\mathrm{M}_{d}$ as a subrepresentation of $\mathrm{M}_{d^{\prime \prime}} \otimes \mathrm{M}_{d^{\prime}}$, with the embedding normalized so as to map the basis vectors $e_{l}^{(d)}$ of Lemma 2.3 to the vectors

$$
\tau_{l}^{\left(d ; d^{\prime}, d^{\prime \prime}\right)}:=F^{l} \cdot \tau_{0}^{\left(d ; d^{\prime}, d^{\prime \prime}\right)}
$$


with $\tau_{0}^{\left(d ; d^{\prime}, d^{\prime \prime}\right)}$ given by the formulas 2.8 and 2.7$)$. We denote the coefficients of these vectors in the tensor product basis by $T_{l ; m}^{l^{\prime}, l^{\prime \prime}}=T_{l ; m}^{l^{\prime}, l^{\prime \prime}}\left(d^{\prime}, d^{\prime \prime}\right)$, so that

$$
\tau_{l}^{\left(d ; d^{\prime}, d^{\prime \prime}\right)}=\sum_{l^{\prime}, l^{\prime \prime}} T_{l ; m}^{l^{\prime}, l^{\prime \prime}} \times\left(e_{l^{\prime \prime}} \otimes e_{l^{\prime}}\right) .
$$

The vectors $\tau_{l}^{\left(d ; d^{\prime}, d^{\prime \prime}\right)}$ also form a basis of $\mathrm{M}_{d^{\prime \prime}} \otimes \mathrm{M}_{d^{\prime}}$.

\section{VARIOUS FORMS OF THE INTEGRAL FUNCTIONS}

In this section, we introduce the functions in terms of which the spin chain - Coulomb gas correspondence is defined and studied. The functions

$$
\rho_{m_{1}, \ldots, m_{n}}^{\left(x_{0}\right)}, \widetilde{\rho}_{m_{1}, \ldots, m_{n}}^{\left(x_{0}\right)}, \varphi_{l_{1}, \ldots, l_{n}}^{\left(x_{0}\right)}, \alpha_{l_{1}, \ldots, l_{j-1} ; l, m ; l_{j+2}, \ldots, l_{n}}^{\left(x_{0}\right)}
$$

of $n$ variables $x_{1}, \ldots, x_{n}$ will be indexed by an anchor point $x_{0} \in \mathbb{R}$ and various $n$-tuples of non-negative integers $m_{j}, l_{j}, l, m \in \mathbb{Z}_{\geq 0}$. All these functions will be defined by integrals of essentially the same multivalued integrand - the differences lie in the choice of the integration surface and the choices of branch and rephasing of the integrand, which are often easiest to indicate by figures. The definition of the correspondence will use $\varphi_{l_{1}, \ldots, l_{n}}^{\left(x_{0}\right)}$ as basis functions, and the other functions are used for proving properties of the correspondence. For this purpose, various properties of the functions and relations among them are stated in this section. Some proofs are postponed to Appendix $\mathrm{A}$.

A parameter $\kappa \in(0, \infty) \backslash \mathbb{Q}$ is fixed throughout, and the deformation parameter $q=e^{\mathfrak{i} \pi 4 / \kappa}$ is chosen. We also fix the number $n \in \mathbb{N}$ of variables, and real parameters $d_{1}, \ldots, d_{n}$, which later in Section 4 will be taken to be dimensions of representations of type $\mathrm{M}_{d}$.

We use the shorthand notation $\boldsymbol{x}=\left(x_{1}, \ldots, x_{n}\right)$ for the arguments of the functions. The domain of definition will be either the chamber $\mathfrak{X}_{n}$ or the restricted chamber $\mathfrak{X}_{n}^{\left(x_{0}\right)}$, given by 1.2 or 1.5 , respectively, so that we always assume the variables ordered according to

$$
x_{0}<x_{1}<\cdots<x_{n} .
$$

For fixed $\boldsymbol{x}$ and $x_{0}$, the value of the function will be written as an integral of Dotsenko-Fateev type [DF84, as in the Coulomb gas formalism of conformal field theory. The integrand is a branch of the following multivalued function, a product of powers of differences,

$$
\begin{aligned}
f^{(\ell)}(\boldsymbol{x} ; \boldsymbol{w}) & =f_{d_{1}, \ldots, d_{n}}^{(\ell)}\left(x_{1}, \ldots, x_{n} ; w_{1}, \ldots, w_{\ell}\right) \\
& =\prod_{1 \leq i<j \leq n}\left(x_{j}-x_{i}\right)^{\frac{2}{\kappa}\left(d_{i}-1\right)\left(d_{j}-1\right)} \prod_{\substack{1 \leq i \leq n \\
1 \leq r \leq \ell}}\left(w_{r}-x_{i}\right)^{-\frac{4}{\kappa}\left(d_{i}-1\right)} \prod_{1 \leq r<s \leq \ell}\left(w_{s}-w_{r}\right)^{\frac{8}{\kappa}},
\end{aligned}
$$

and the auxiliary variables $w_{1}, \ldots, w_{\ell}$ are to be integrated over. More precisely, the integrand will be defined on some simply connected subset of

$$
\mathfrak{W}^{(\ell)}=\mathfrak{W}_{x_{1}, \ldots, x_{n}}^{(\ell)}:=\left\{\left(w_{1}, \ldots, w_{\ell}\right) \in\left(\mathbb{C} \backslash\left\{x_{1}, \ldots, x_{n}\right\}\right)^{\ell} \mid w_{r} \neq w_{s} \text { for all } r \neq s\right\} .
$$

Remark 3.1. The logarithmic differential of the multivalued function $f^{(\ell)}$ is the single-valued one-form

$$
\mathrm{d}\left(\log \left(f^{(\ell)}(\boldsymbol{x} ; \boldsymbol{w})\right)\right)=\sum_{r=1}^{\ell}\left(\sum_{i=1}^{n} \frac{4\left(1-d_{i}\right) / \kappa}{w_{r}-x_{i}}+\sum_{s \neq r} \frac{8 / \kappa}{w_{r}-w_{s}}\right) \mathrm{d} w_{r} .
$$

Thus, to define a branch of the integrand on a simply connected subset of $\mathfrak{W}^{(\ell)}$, it is sufficient to give its value at some point $\boldsymbol{w}^{\prime}$, and then set

$$
f_{\text {branch }}(\boldsymbol{x} ; \boldsymbol{w})=f_{\text {branch }}\left(\boldsymbol{x} ; \boldsymbol{w}^{\prime}\right) \times \exp \left(\int_{\boldsymbol{w}^{\prime}}^{\boldsymbol{w}} \mathrm{d}\left(\log \left(f^{(\ell)}(\boldsymbol{x} ; \cdot)\right)\right)\right),
$$


where the path of integration from $\boldsymbol{w}^{\prime}$ to $\boldsymbol{w}$ stays in the simply connected subset.

We will frequently partition the variables $w_{1}, \ldots, w_{\ell}$ to $n$ subsets of sizes $m_{1}, \ldots, m_{n}$, in which case we use the notation

$$
I^{(i)}=I_{m_{1}, \ldots, m_{n}}^{(i)}=\left\{r \in \mathbb{Z} \mid \sum_{j=1}^{i} m_{j} \geq r>\sum_{j=1}^{i-1} m_{j}\right\} \quad(i=1, \ldots, n)
$$

for the partition of the indices.

3.1. Real integral functions as integrals over a product of simplices. The integrand (3.1) has a constant phase on the following simply connected real subset of $\mathfrak{W}^{(\ell)}$, a product of simplices of dimensions $m_{1}, \ldots, m_{n}$ with $\sum_{i=1}^{n} m_{i}=\ell$,

$$
\begin{aligned}
& \mathcal{R}_{m_{1}, \ldots, m_{n}}=\left\{\left(w_{1}, \ldots, w_{\ell}\right) \in \mathbb{R}^{\ell} \mid x_{0}<w_{1}<w_{2}<w_{3}<\cdots<w_{m_{1}}<x_{1},\right. \\
& x_{1}<w_{m_{1}+1}<\cdots<w_{m_{1}+m_{2}}<x_{2}, \\
& \left.x_{n-1}<w_{m_{1}+\cdots+m_{n-1}+1}<\cdots<w_{m_{1}+\cdots+m_{n}}<x_{n}\right\} .
\end{aligned}
$$

We define the real-valued functions $\rho_{m_{1}, \ldots, m_{n}}^{\left(x_{0}\right)}: \mathfrak{X}^{\left(x_{0}\right)} \rightarrow \mathbb{R}$ as integrals over this set

$$
\rho_{m_{1}, \ldots, m_{n}}^{\left(x_{0}\right)}(\boldsymbol{x}):=\int_{\mathcal{R}_{m_{1}, \ldots, m_{n}}}\left|f^{(\ell)}(\boldsymbol{x} ; \boldsymbol{w})\right| \mathrm{d} w_{1} \cdots \mathrm{d} w_{\ell} .
$$

In applications, it is usually desirable to write the final results in terms of these functions, because of their transparent definition and real-valuedness.

The integrals $\rho_{m_{1}, \ldots, m_{n}}^{\left(x_{0}\right)}(\boldsymbol{x})$ are convergent for large enough $\kappa$ - the precise condition is

$$
\kappa>4 \times\left(\max _{1 \leq i \leq n} d_{i}-1\right),
$$

and this will often be implicitly assumed. Nevertheless, our main results are valid for all irrational positive $\kappa$ - they are obtained by meromorphic analytic continuation in $\kappa$. Indeed, the analytic continuation of the real integrals $\rho_{m_{1}, \ldots, m_{n}}^{\left(x_{0}\right)}(\boldsymbol{x})$ can be done by regularizing the divergent integrals, as discussed in JJK16. If such a regularization is performed by the method of counterterms, one can see, in principle explicitly, that for $\Re \mathfrak{e}(\kappa)>0$ the only singularities are isolated poles at some rational values of $\kappa$. For concrete examples of the analytic continuation and poles, $\rho_{m_{1}}^{\left(x_{0}\right)}\left(x_{1}\right)$ and $\rho_{0, m_{2}}^{\left(x_{0}\right)}\left(x_{1}, x_{2}\right)$ can be reduced to the Selberg integral given in Remark 3.9

3.2. Integrals over a product of deformed hypercubes. It is natural to extend the integrand above from the real submanifold $\mathcal{R}_{m_{1}, \ldots, m_{n}} \subset \mathfrak{W}^{(\ell)}$ to an open subset. A convenient choice for intermediate manipulations is the simply connected subset

$$
\begin{aligned}
\widetilde{\mathfrak{W}}_{m_{1}, \ldots, m_{n}}:=\left\{\boldsymbol{w} \in \mathfrak{W}^{(\ell)} \mid\right. & \forall i \forall r \in I^{(i)}: \Re \mathfrak{e}\left(x_{i-1}\right)<\Re \mathfrak{e}\left(w_{r}\right)<\Re \mathfrak{e}\left(x_{i}\right), \\
& \left.\forall i \forall r, s \in I^{(i)}, r<s: w_{s}-w_{r} \in \mathbb{C} \backslash \mathfrak{i} \mathbb{R}_{+}\right\} .
\end{aligned}
$$

On $\widetilde{\mathfrak{W}}_{m_{1}, \ldots, m_{n}}$, we choose a branch of the multivalued function $f^{(\ell)}(\boldsymbol{x} ; \cdot)$ of $(3.1)$, and rephase it so that it becomes real and positive on $\mathcal{R}_{m_{1}, \ldots, m_{n}} \subset \widetilde{\mathfrak{W}}_{m_{1}, \ldots, m_{n}}$. This function

$$
f_{m_{1}, \ldots, m_{n}}(\boldsymbol{x} ; \cdot): \widetilde{\mathfrak{W}}_{m_{1}, \ldots, m_{n}} \rightarrow \mathbb{C}
$$




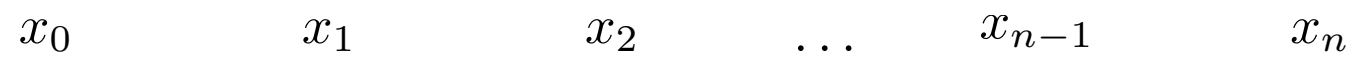

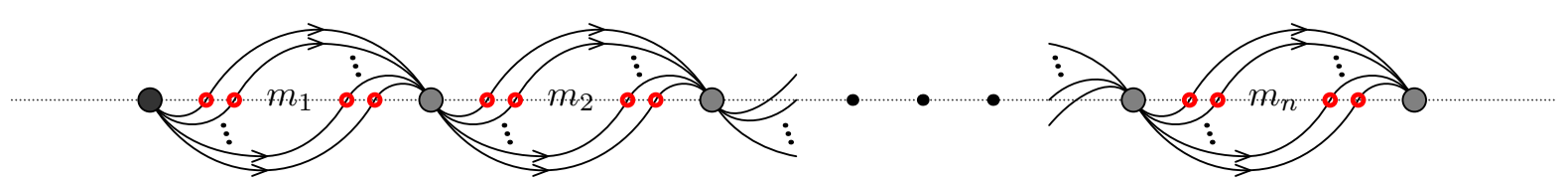

FIGURE 3.1. The integration surface $\widetilde{\mathcal{R}}_{m_{1}, \ldots, m_{n}}$ is a Cartesian product of deformed hypercubes of dimensions $m_{1}, \ldots, m_{n}$. The point $w_{1}<\cdots<w_{\ell}$, where the integrand $f \widetilde{\tilde{m}}_{1}, \ldots, m_{n}(\boldsymbol{x} ; \cdot)$ is rephased to be positive, is marked by red circles. For $r, s \in I^{(i)}, s<r$, the integration path of $w_{r}$ thus remains below the path of $w_{s}$.

can be defined for example using Remark 3.1, i.e., by fixing a point $\boldsymbol{w}^{\prime} \in \mathcal{R}_{m_{1}, \ldots, m_{n}}$, setting the value at that point equal to the absolute value of $f^{(\ell)}\left(\boldsymbol{x} ; \boldsymbol{w}^{\prime}\right)$, and analytically continuing by integrating the single-valued logarithmic differential.

In particular, we can write the real integral function $\rho_{m_{1}, \ldots, m_{n}}^{\left(x_{0}\right)}$ as an integral of $f \approx(\boldsymbol{x} ; \cdot)$

$$
\rho_{m_{1}, \ldots, m_{n}}^{\left(x_{0}\right)}(\boldsymbol{x})=\int_{\mathcal{R}_{m_{1}, \ldots, m_{n}}} f_{m_{1}, \ldots, m_{n}}^{\approx}\left(\boldsymbol{x} ; w_{1}, \ldots, w_{\ell}\right) \mathrm{d} w_{1} \cdots \mathrm{d} w_{\ell}
$$

It will instead be easier to express the basis functions of our correspondence in terms of the closely related function

$$
\widetilde{\rho}_{m_{1}, \ldots, m_{n}}^{\left(x_{0}\right)}(\boldsymbol{x}):=\int_{\widetilde{\mathcal{R}}_{m_{1}, \ldots, m_{n}}} f_{m_{1}, \ldots, m_{n}}^{\approx}\left(\boldsymbol{x} ; w_{1}, \ldots, w_{\ell}\right) \mathrm{d} w_{1} \cdots \mathrm{d} w_{\ell}
$$

where the integration surface $\widetilde{\mathcal{R}}_{m_{1}, \ldots, m_{n}} \subset \widetilde{\mathfrak{W}}_{m_{1}, \ldots, m_{n}}$ is such that for any $r \in I^{(i)}$, the variable $w_{r}$ is integrated from $x_{i-1}$ to $x_{i}$. In view of the definition of the simply connected set $\widetilde{\mathfrak{W}}_{m_{1}, \ldots, m_{n}}$, this unambiguously determines the homotopy type of the integration surface $\widetilde{\mathcal{R}}_{m_{1}, \ldots, m_{n}}$, and consequently the function $\widetilde{\rho}_{m_{1}, \ldots, m_{n}}^{\left(x_{0}\right)}(\boldsymbol{x})$. Figure 3.1 illustrates how the variables turn around each other in this integration, and indicates the choice of a point where the integrand is rephased to be positive.

Lemma 3.2. The function

$$
\widetilde{\rho}_{m_{1}, \ldots, m_{n}}^{\left(x_{0}\right)}: \mathfrak{X}_{n}^{\left(x_{0}\right)} \rightarrow \mathbb{C}
$$

is related to the real integral function (3.3) by

$$
\widetilde{\rho}_{m_{1}, \ldots, m_{n}}^{\left(x_{0}\right)}(\boldsymbol{x})=\left(\prod_{i=1}^{n} q^{-\left(\begin{array}{c}
m_{i} \\
2
\end{array}\right)}\left[m_{i}\right] !\right) \times \rho_{m_{1}, \ldots, m_{n}}^{\left(x_{0}\right)}(\boldsymbol{x}), \quad \text { for } \boldsymbol{x} \in \mathfrak{X}_{n}^{\left(x_{0}\right)} .
$$

The proof is a straightforward contour deformation argument, decomposing the contour in (3.5) to $\prod_{i=1}^{n}\left(m_{i} !\right)$ pieces. We postpone the details of the proof to Appendix A

3.3. Basis functions as integrals over families of non-intersecting loops. Let us now define the integrals $\varphi_{l_{1}, \ldots, l_{n}}^{\left(x_{0}\right)}$ which will serve as our basis functions in defining the spin chain - Coulomb gas correspondence. The integration surfaces are certain families of non-intersecting loops used also in [FW91]. In contrast to the earlier integrals $\rho_{m_{1}, \ldots, m_{n}}^{\left(x_{0}\right)}$ and $\widetilde{\rho}_{m_{1}, \ldots, m_{n}}^{\left(x_{0}\right)}$, for the convergence of these integrals it suffices that $\Re \mathfrak{e}(\kappa)>0$. In the choices of indices, we try to consistently use $l_{j} \in \mathbb{Z}_{\geq 0}$ for numbers of loops, and $m_{j} \in \mathbb{Z}_{\geq 0}$ for multiplicities of paths.

Let

$$
l_{1}, \ldots, l_{n} \in \mathbb{Z}_{\geq 0}, \quad \ell=\sum_{i=1}^{n} l_{i}
$$




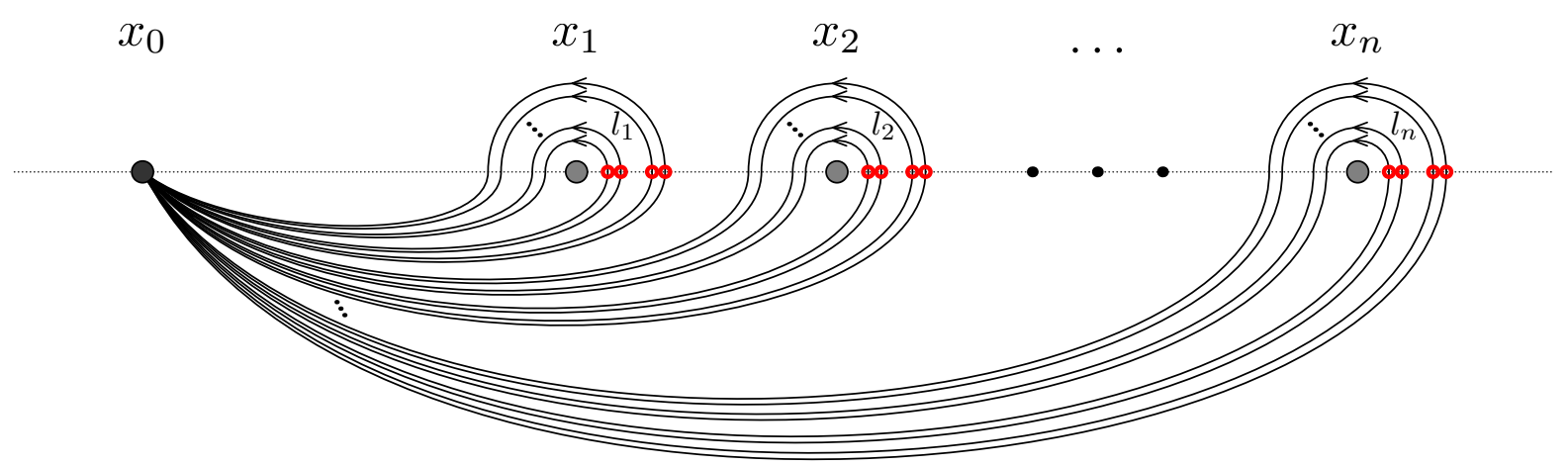

FIGURE 3.2. The integration surface $\mathfrak{L}_{l_{1}, \ldots, l_{n}}^{\ni}$. The point where the integrand $f_{l_{1}, \ldots, l_{n}}^{\ni}(\boldsymbol{x} ; \cdot)$ is rephased to be positive is marked by red circles.

and define the partition $\left(I_{l_{1}, \ldots, l_{n}}^{(i)}\right)_{i=1}^{n}$ of the indices of the $w$-variables as in $(3.2)$, now with parts of sizes $l_{1}, \ldots, l_{n}$. The integration surface $\mathfrak{L}_{l_{1}, \ldots, l_{n}}$ - a family of non-intersecting loops illustrated in Figure 3.2 — and the associated integrand $f_{l_{1}, \ldots, l_{n}}^{\ni}$ are defined as follows:

- Each of the variables $w_{r}$, for $r \in I^{(i)}$, makes a simple loop in $\mathbb{C} \backslash\left\{x_{1}, \ldots, x_{n}\right\}$ starting and ending at the anchor point $x_{0}$, and encircling the point $x_{i}$ once in the positive direction. The loop of $w_{r}$, for $r \in I^{(i)}$, must never cross the lines $x_{j}+\mathfrak{i} \mathbb{R}_{+}$for $j \neq i$, nor the lines $w_{s}+\mathfrak{i} \mathbb{R}_{+}$for $s \in I^{(j)}$ with $j<i$.

- The $l_{i}$ loops around $x_{i}$ are nested in such a way that if $r, s \in I^{(i)}$ and $r<s$, then the loop of $w_{s}$ encircles the loop of $w_{r}$.

- There is a point $\boldsymbol{w}^{\prime}=\left(w_{1}^{\prime}, \ldots, w_{\ell}^{\prime}\right) \in \mathfrak{L}_{l_{1}, \ldots, l_{n}}^{\ni}$, also illustrated in Figure 3.2 such that

$$
\begin{gathered}
x_{1}<w_{1}^{\prime}<w_{2}^{\prime}<w_{3}^{\prime}<\cdots<w_{l_{1}}^{\prime}<x_{2}, \\
x_{2}<w_{l_{1}+1}^{\prime}<w_{l_{1}+2}^{\prime}<\cdots<w_{l_{1}+l_{2}}^{\prime}<x_{3}, \\
\vdots \\
x_{n}<w_{l_{1}+\cdots+l_{n-1}+1}^{\prime}<\cdots<w_{l_{1}+\cdots+l_{n}}^{\prime} .
\end{gathered}
$$

As the integrand, we choose a branch and rephasing of the function $f^{(\ell)}(\boldsymbol{x} ; \cdot)$ of $[3.1$, so as to make it positive at $\boldsymbol{w}^{\prime}$. Such an integrand

$$
f_{l_{1}, \ldots, l_{n}}^{\ni}(\boldsymbol{x} ; \cdot): \mathfrak{L}_{l_{1}, \ldots, l_{n}}^{\ni} \rightarrow \mathbb{C}
$$

can again be defined using the single-valued logarithmic differential as explained in Remark 3.1 .

The integrals of $f^{\ni}$ over $\mathfrak{L}^{\ni}$,

$$
\varphi_{l_{1}, \ldots, l_{n}}^{\left(x_{0}\right)}(\boldsymbol{x})=\int_{\mathfrak{L}_{l_{1}, \ldots, l_{n}}^{\ni}} f_{l_{1}, \ldots, l_{n}}^{\ni}\left(\boldsymbol{x} ; w_{1}, \ldots, w_{\ell}\right) \mathrm{d} w_{1} \cdots \mathrm{d} w_{\ell}
$$

define functions

$$
\varphi_{l_{1}, \ldots, l_{n}}^{\left(x_{0}\right)}: \mathfrak{X}_{n}^{\left(x_{0}\right)} \rightarrow \mathbb{C}
$$

The transformation rules of these functions under translation and scaling are the following.

Lemma 3.3. For any $\xi \in \mathbb{R}$, we have

$$
\varphi_{l_{1}, \ldots, l_{n}}^{\left(x_{0}+\xi\right)}\left(x_{1}+\xi, \ldots, x_{n}+\xi\right)=\varphi_{l_{1}, \ldots, l_{n}}^{\left(x_{0}\right)}\left(x_{1}, \ldots, x_{n}\right),
$$


and for any $\lambda>0$ we have

$$
\varphi_{l_{1}, \ldots, l_{n}}^{\left(\lambda x_{0}\right)}\left(\lambda x_{1}, \ldots, \lambda x_{n}\right)=\lambda^{\Delta^{d_{1}, \ldots, d_{n}}(\ell)} \times \varphi_{l_{1}, \ldots, l_{n}}^{\left(x_{0}\right)}\left(x_{1}, \ldots, x_{n}\right),
$$

where $\ell=\sum_{i=1}^{n} l_{i}$ and

$$
\Delta^{d_{1}, \ldots, d_{n}}(\ell)=\frac{2}{\kappa} \sum_{i<j}\left(d_{i}-1\right)\left(d_{j}-1\right)-\frac{4}{\kappa} \ell \sum_{i}\left(d_{i}-1\right)+\frac{8}{\kappa} \frac{\ell(\ell-1)}{2}+\ell .
$$

Proof. For the first statement, make the changes of variables $u_{r}=w_{r}+\xi$ in the integrals. For the second, make the change of variables $u_{r}=\lambda w_{r}$, and notice that the integrand has the scaling

$$
f_{l_{1}, \ldots, l_{n}}^{\ni}(\lambda \boldsymbol{x} ; \lambda \boldsymbol{w})=\lambda^{\frac{2}{\kappa} \sum_{i<j}\left(d_{i}-1\right)\left(d_{j}-1\right)-\frac{4}{\kappa} \ell \sum_{i}\left(d_{i}-1\right)+\frac{8}{\kappa} \frac{\ell(\ell-1)}{2}} \times f_{l_{1}, \ldots, l_{n}}^{\ni}(\boldsymbol{x} ; \boldsymbol{w}),
$$

and that the Jacobian of the change of variables in the $\ell$-dimensional integral is $\lambda^{\ell}$.

As another simple remark, we record the fact that when $d_{i}=1$ for some $i$, so that the trivial representation $M_{1}$ is a factor in the tensor product $\bigotimes_{j} M_{d_{j}}$, then the function is actually independent of the corresponding variable.

Lemma 3.4. If $d_{i}=1$ for some $i \in\{1, \ldots, n\}$, then for any fixed values of the other variables $x_{j}, j \neq i$, the function $\varphi_{l_{1}, \ldots, l_{n}}^{\left(x_{0}\right)}$ is constant as a function of the variable $x_{i}$.

Proof. For two values of the variable $x_{i}$, one can choose the integration surface $\mathfrak{L}_{l_{1}, \ldots, l_{n}}^{\ni}$ to be the same, in such a way as to obtain the correct homotopy type for both cases. If $d_{i}=1$, the integrand $f_{l_{1}, \ldots, l_{n}}^{\ni}(\boldsymbol{x} ; \boldsymbol{w})$ is constant as a function of $x_{i}$, because all the exponents of differences in which $x_{i}$ appears are proportional to $d_{i}-1$. Therefore, for two values of the variable $x_{i}$, the values of the function $\varphi_{l_{1}, \ldots, l_{n}}^{\left(x_{0}\right)}$ are in fact given by the same integral.

The basis functions $\varphi_{l_{1}, \ldots, l_{n}}^{\left(x_{0}\right)}$ can be decomposed to linear combinations of the real integrals $\rho_{m_{1}, \ldots, m_{n}}^{\left(x_{0}\right)}$. We begin with the simplest case of one point, $n=1$. Even this simple case will have an important consequence, Lemma 3.8 .

Lemma 3.5. Consider the case $n=1$, and denote $d_{1}=d \in \mathbb{Z}_{>0}, l_{1}=\ell, x_{1}=x$. Then we have

$$
\varphi_{\ell}^{\left(x_{0}\right)}(x)=\left([\ell] ! \prod_{m=1}^{\ell}\left(q^{d-m}-q^{m-d}\right)\right) \times \rho_{\ell}^{\left(x_{0}\right)}(x) .
$$

In particular, $\varphi_{\ell}^{\left(x_{0}\right)}$ is identically zero if $\ell \geq d$.

The proof is a straightforward contour manipulation argument, whose details are left to Appendix A In the next lemma, we write the basis functions $\varphi^{\left(x_{0}\right)}$ in terms of the integrals $\widetilde{\rho}^{\left(x_{0}\right)}$, in the case $n=2$. The integrals $\widetilde{\rho}^{\left(x_{0}\right)}$, in turn, could be written in terms of the real integrals $\rho^{\left(x_{0}\right)}$ by Lemma 3.2 . We use this result several times in the course of proving the main theorems: as such in Lemma 4.1, and in small variations in Lemma 4.2 and Proposition 4.5

Lemma 3.6. Let $d_{1}, d_{2} \in \mathbb{Z}_{>0}$ and $0 \leq l_{1}<d_{1}, 0 \leq l_{2}<d_{2}$. Then we have

$$
\begin{aligned}
\varphi_{l_{1}, l_{2}}^{\left(x_{0}\right)}\left(x_{1}, x_{2}\right)=q^{\left(\begin{array}{c}
l_{1} \\
2
\end{array}\right)+\left(\begin{array}{c}
l_{2} \\
2
\end{array}\right)\left(q-q^{-1}\right)^{l_{1}+l_{2}} \frac{\left[d_{1}-1\right] !\left[d_{2}-1\right] !}{\left[d_{1}-l_{1}-1\right] !\left[d_{2}-l_{2}-1\right] !}} \\
\times \sum_{m=0}^{l_{2}} q^{m\left(m-l_{2}+d_{1}-1\right)}\left[\begin{array}{c}
l_{2} \\
m
\end{array}\right] \widetilde{\rho}_{l_{1}+m, l_{2}-m}^{\left(x_{0}\right)}\left(x_{1}, x_{2}\right) .
\end{aligned}
$$

The proof is postponed to Appendix A

In the case of general $n$, the basis functions $\varphi^{\left(x_{0}\right)}$ can still be written, in principle explicitly, in terms of the real integrals $\rho^{\left(x_{0}\right)}$. The proof of our main results, however, do not rely on this explicit formula. 
Lemma 3.7. We have

$$
\varphi_{l_{1}, \ldots, l_{n}}^{\left(x_{0}\right)}(\boldsymbol{x})=\sum_{m_{1}, \ldots, m_{n}} C_{l_{1}, \ldots, l_{n}}^{m_{1}, \ldots, m_{n}} \times \widetilde{\rho}_{m_{1}, \ldots, m_{n}}^{\left(x_{0}\right)}(\boldsymbol{x}),
$$

with some coefficients $C_{l_{1}, \ldots, l_{n}}^{m_{1}, \ldots, m_{n}}$, which are zero unless $\sum_{i} l_{i}=\sum_{i} m_{i}$ and $\sum_{i=1}^{j} l_{i} \leq \sum_{i=1}^{j} m_{i}$ for all $j=1, \ldots, n$.

The proof is sketched in Appendix A.

The following important particular case already follows from the simple calculations performed in the proof of Lemma 3.5 .

Lemma 3.8. Whenever $l_{i} \geq d_{i}$ for some $i=1, \ldots, n$, we have

$$
\varphi_{l_{1}, \ldots, l_{n}}^{\left(x_{0}\right)}\left(x_{1}, \ldots, x_{n}\right) \equiv 0 .
$$

The short proof is given in Appendix $\mathrm{A}$

3.4. Mixed integral functions for asymptotics. To extract the asymptotic behavior of our functions as $\left|x_{j+1}-x_{j}\right| \rightarrow 0$, we rewrite them in yet another way. We still keep $d_{1}, \ldots, d_{n}$ fixed, and for any

$$
l_{1}, \ldots, l_{j-1}, l_{j+2}, \ldots, l_{n} \in \mathbb{Z}_{\geq 0}, \quad l \in \mathbb{Z}_{\geq 0}, \quad m \in \mathbb{Z}_{\geq 0}
$$

we define an integration surface

$$
\mathfrak{M}_{l_{1}, \ldots, l_{j-1} ; l, m ; l_{j+2}, \ldots, l_{n}}^{\Im},
$$

rephased integrand

$$
f_{l_{1}, \ldots, l_{j-1} ; l, m ; l_{j+2}, \ldots, l_{n}}^{\Im}(\boldsymbol{x} ; \boldsymbol{w}),
$$

and an integral function

$$
\alpha_{l_{1}, \ldots, l_{j-1} ; l, m ; l_{j+2}, \ldots, l_{n}}^{\left(x_{0}\right)}(\boldsymbol{x})
$$

as follows. The surface $\mathfrak{M}^{\curvearrowright}$ is a mixture between a family of non-intersecting loops (see Section 3.3 ) and a deformed hypercube (see Section 3.2) as illustrated in Figure 3.3. Trusting that the figure is sufficiently similar to the earlier ones, we content ourselves to give the following slightly informal descriptions:

- There are $m$ variables integrated from $x_{j}$ to $x_{j+1}$, and they turn around each other like in the deformed hypercube integrals of Section 3.2 .

- For $i \notin\{j, j+1\}$ there are $l_{i}$ loops around $x_{i}$ starting and ending at the anchor point $x_{0}$, and they are nested and turning around each other like in the earlier families of non-intersecting loops of Section 3.3 .

- There are $l$ loops around the entire paths of the deformed hypercube integrals from $x_{j}$ to $x_{j+1}$, and they are nested and turning around each other like in the families of non-intersecting loops, as if the entire deformed hypercube would be a single point.

- The rephasing and branch choice of the integrand is such that $f^{\curvearrowright}$ is positive at a point $\boldsymbol{w}^{\prime}$ illustrated by the red circles in Figure 3.3

The integral function $\alpha^{\left(x_{0}\right)}$ is then defined as

$$
\alpha_{l_{1}, \ldots, l_{j-1} ; l, m ; l_{j+2}, \ldots, l_{n}}^{\left(x_{0}\right)}(\boldsymbol{x}):=q^{\left(\begin{array}{c}
m \\
2
\end{array}\right)} \frac{1}{[m] !} \times \int_{\mathfrak{M}_{l_{1}, \ldots, i, m ;, \ldots, l_{n}}} f_{l_{1}, \ldots, ; l, m ;, \ldots, l_{n}}^{\curvearrowright}(\boldsymbol{x} ; \boldsymbol{w}) \mathrm{d} \boldsymbol{w} .
$$

These, just like the integrals $\widetilde{\rho}$, are convergent for large enough $\kappa$, namely $\kappa>4 \times\left(\max \left(d_{j}, d_{j+1}\right)-1\right)$. They can also be analytically continued in $\kappa$. The prefactor is included to make the integrals more closely related to the integrals over a real simplex — compare with Lemma 3.2 


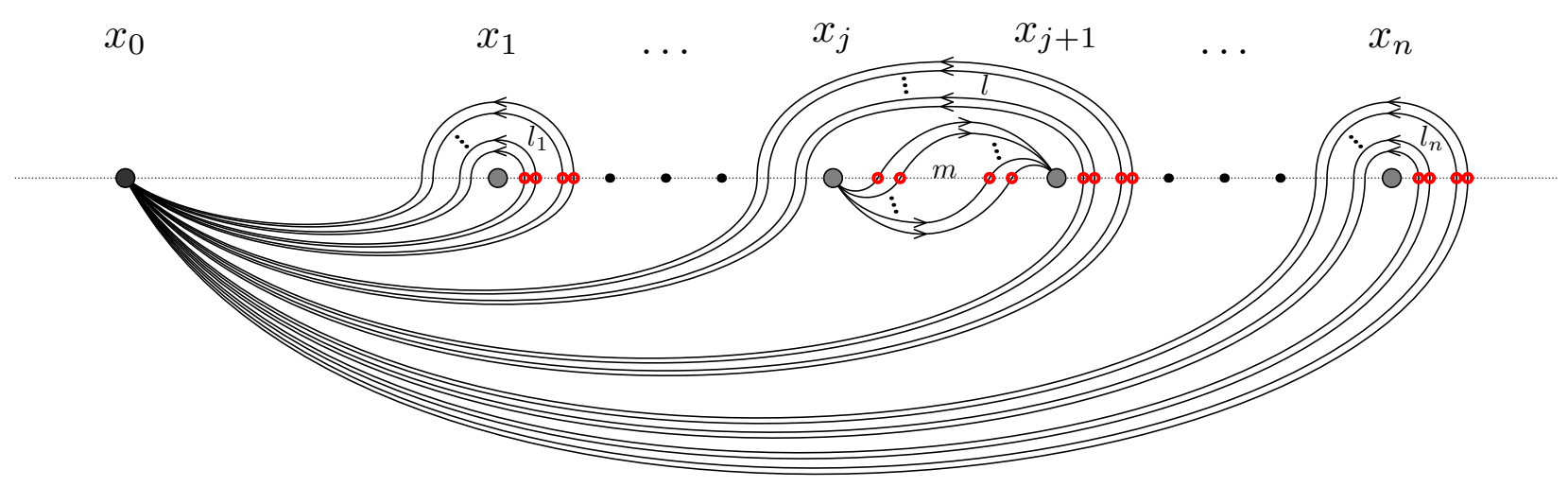

FIGURE 3.3. The integration surface $\mathfrak{M}_{l_{1}, \ldots ; l, m ; \ldots, l_{n}}^{\curvearrowright}$ is used to construct functions that have an explicit asymptotic behavior as $\left|x_{j+1}-x_{j}\right| \rightarrow 0$. The point where the integrand $f \sim_{l_{1}, \ldots ; l, m ; \ldots, l_{n}}(\boldsymbol{x} ; \cdot)$ is rephased to be positive is marked by red circles.

In order to state the results about the asymptotics, we use the exponents

$$
\Delta_{d}^{d^{\prime}, d^{\prime \prime}}:=\frac{2\left(1+d^{2}-\left(d^{\prime}\right)^{2}-\left(d^{\prime \prime}\right)^{2}\right)+\kappa\left(d^{\prime}+d^{\prime \prime}-d-1\right)}{2 \kappa},
$$

and define the multiplicative constants $B_{d}^{d^{\prime}, d^{\prime \prime}}$ for $\kappa>4 \times\left(\max \left\{d^{\prime}, d^{\prime \prime}\right\}-1\right)$ by the convergent integral over an $m$-dimensional simplex, with $m=\frac{1}{2}\left(d^{\prime}+d^{\prime \prime}-1-d\right)$,

$$
B_{d}^{d^{\prime}, d^{\prime \prime}}:=\int_{0}^{1} \mathrm{~d} w_{1} \int_{w_{1}}^{1} \mathrm{~d} w_{2} \cdots \int_{w_{m-1}}^{1} \mathrm{~d} w_{m} \prod_{r=1}^{m} w_{r}^{-\frac{4}{\kappa}\left(d^{\prime}-1\right)} \prod_{r=1}^{m}\left(1-w_{r}\right)^{-\frac{4}{\kappa}\left(d^{\prime \prime}-1\right)} \prod_{1 \leq r<s \leq m}\left(w_{s}-w_{r}\right)^{\frac{8}{\kappa}} .
$$

For general values of $\kappa$, the multiplicative constants $B_{d}^{d^{\prime}, d^{\prime \prime}}$ are defined by the analytic continuation of this generalized beta-function.

Remark 3.9. The multiplicative constant (3.8) is trivial in the case $d=d^{\prime}+d^{\prime \prime}-1$ (that is $m=0$ ): we then have $B_{d}^{d^{\prime}, d^{\prime \prime}}=1$. In the case $d=d^{\prime}+d^{\prime \prime}-3$ (that is $m=1$ ), the multiplicative constants (3.8) are ordinary beta-functions. In general, (3.8) is a Selberg integral, which can also be evaluated in terms of gamma-functions [Sel44, DF85, For10]:

$$
B_{d}^{d^{\prime}, d^{\prime \prime}}=\frac{1}{m !} \prod_{p=1}^{m} \frac{\Gamma\left(1-\frac{4}{\kappa}\left(d^{\prime}-p\right)\right) \Gamma\left(1-\frac{4}{\kappa}\left(d^{\prime \prime}-p\right)\right) \Gamma\left(1+\frac{4}{\kappa} p\right)}{\Gamma\left(1+\frac{4}{\kappa}\right) \Gamma\left(2-\frac{4}{\kappa}\left(d^{\prime}+d^{\prime \prime}-m-p\right)\right)} .
$$

This expression gives explicitly the analytic continuation in $\kappa$, and it in particular shows that poles and zeroes may only occur in $B_{d}^{d^{\prime}, d^{\prime \prime}}$ when $\kappa$ tends to particular rational values.

Remark 3.10. In terms of the Kac labeled conformal weights (1.1), one may express the overall homogeneity degree of $\varphi_{l_{1}, \ldots, l_{n}}^{\left(x_{0}\right)}$, given in Lemma 3.3, as

$$
\Delta^{d_{1}, \ldots, d_{n}}(\ell)=\Delta_{d}^{d_{1}, \ldots, d_{n}}=h_{1, d}-\sum_{i=1}^{n} h_{1, d_{i}},
$$

where $d=\sum_{i=1}^{n} d_{i}-n+1-2 \ell$ can be understood as one of the dimensions of the irreducible subrepresentations of the tensor product $\bigotimes_{i} \mathrm{M}_{d_{i}}$. We furthermore note that the exponents (3.7) are of this form,

$$
\Delta^{d_{1}, d_{2}}(\ell)=\Delta_{d}^{d_{1}, d_{2}} \quad \text { with } \quad d=d_{1}+d_{2}-1-2 \ell
$$


We now show an asymptotics property, which is particularly simple for the functions $\alpha^{\left(x_{0}\right)}$. This will be used later, in Proposition 4.4, to establish the general asymptotics statement.

Lemma 3.11. As $x_{j}$ and $x_{j+1}$ tend to a common limit $\xi$ (with $x_{j-1}<\xi<x_{j+2}$ ), we have

$$
\frac{\alpha_{l_{1}, \ldots, l_{j-1} ; l, m ; l_{j+2}, \ldots, l_{n}}^{\left(x_{0}\right)}\left(x_{1}, \ldots, x_{n}\right)}{\left(x_{j+1}-x_{j}\right)^{\Delta}} \longrightarrow B \times \varphi_{l_{1}, \ldots, l_{j-1}, l, l_{j+2}, \ldots, l_{n}}^{\left(x_{0}\right)}\left(x_{1}, \ldots, x_{j-1}, \xi, x_{j+2}, \ldots, x_{n}\right),
$$

where $d=d_{j}+d_{j+1}-1-2 m$, the exponent $\Delta$ and the constant $B$ are given by

$$
\Delta=\Delta_{d}^{d_{j}, d_{j+1}} \quad \text { and } \quad B=B_{d}^{d_{j}, d_{j+1}},
$$

and $\varphi^{\left(x_{0}\right)}$ on the right hand side is the basis function of $n-1$ points associated to the choice of dimensions $d_{1}, \ldots, d_{j-1}, d, d_{j+2}, \ldots, d_{n}$.

Proof. As a warm-up, note that by a simple scaling (compare with Lemma 3.3 , the integrals $\widetilde{\rho}_{0, m}^{\left(x_{0}\right)}\left(x_{1}, x_{2}\right)$ over deformed hypercubes have the behavior

$$
q^{\left(\begin{array}{c}
m \\
2
\end{array}\right)} \frac{1}{[m] !} \times \widetilde{\rho}_{0, m}^{\left(x_{0}\right)}\left(x_{1}, x_{2}\right)=\rho_{0, m}^{\left(x_{0}\right)}\left(x_{1}, x_{2}\right)=\left(x_{2}-x_{1}\right)^{\Delta_{d}^{d_{1}, d_{2}}} \times B_{d}^{d_{1}, d_{2}},
$$

for $\kappa$ large enough so that both sides are given by convergent integrals. In the general case, the same idea is used with the dominated convergence theorem applied to a subset of the integration variables.

Consider the factors in the integrand $f_{l_{1}, \ldots, i, m ;, \ldots, l_{n}}^{\rightarrow}(\boldsymbol{x} ; \boldsymbol{w})$ that involve $x_{j}$ or $x_{j+1}$, or any of the $m$ variables integrated over the deformed hypercube in $\mathfrak{M}_{l_{1}, \ldots, l, m ;, \ldots, l_{n}}^{\Im}$. The integral over these $m$ variables, divided by $\left|x_{j+1}-x_{j}\right|^{\Delta}$, tends to the integrand of the function $\varphi_{l_{1}, \ldots, l_{j-1}, l, l_{j+2}, \ldots, l_{n}}^{\left(x_{n}\right)}\left(x_{1}, \ldots, x_{j-1}, \xi, x_{j+2}, \ldots, x_{n}\right)$, times $B$. Notice that the other integration contours remain bounded away from these points, so dominated convergence theorem gives the asserted limit.

To conclude, note that both sides of the asserted formula are analytic in $\kappa$.

\section{The Spin Chain - Coulomb gas CORRESPondence}

In this section, we define the spin chain - Coulomb gas correspondence, and show how the representation theoretical properties are translated to properties of the functions. As indicated in the introduction, the underlying idea in our construction is Euler integral solutions to partial differential equations. Informally, the effect of the quantum group is to act on the functions of Section 3 by modifying the integration surfaces and branch choices of the integrand.

The basic ingredients of Euler integral solutions are the closedness of the integration surface, which is the content of Sections 4.4 and 4.5 , and the exactness of the form where a differential operator acts on the integrand, which is covered in Section 4.6. The asymptotics properties, which are crucial in applications for handling boundary conditions, are analyzed in Section 4.3 . Möbius covariance is treated in Section 4.7. In summary, by a succession of small steps, which combine formulas for the representations of the quantum group given in Section 2 with properties of the functions established in Section 3 , in the end of this section we will have proven the main result, whose precise formulation is given in Theorems 4.16 and 4.17 in Section 4.8 .

From Section 2, we use in particular the $d$-dimensional irreducible representation $\mathrm{M}_{d}$ of the quantum group $\mathcal{U}_{q}\left(\mathfrak{s l}_{2}\right)$, defined in Lemma 2.3 Its basis $\left(e_{l}^{(d)}\right)_{l=0,1, \ldots, d-1}$, introduced in the same lemma, is also used below. For simplicity of notation, we often omit the superscript reference to the dimension $d$. 
4.1. Definition of the correspondence. Fix $d_{1}, \ldots, d_{n} \in \mathbb{Z}_{>0}$. Denote by $\mathrm{M}_{d_{i}}$ the $d_{i}$-dimensional irreducible representation of $\mathcal{U}_{q}\left(\mathfrak{s l}_{2}\right)$ defined in Section 2.2.2. Consider the tensor product representation

$$
\bigotimes_{i=1}^{n} \mathrm{M}_{d_{i}}=\mathrm{M}_{d_{n}} \otimes \mathrm{M}_{d_{n-1}} \otimes \cdots \otimes \mathrm{M}_{d_{2}} \otimes \mathrm{M}_{d_{1}} .
$$

The order of tensorands will always be as shown on the right hand side, but for brevity we usually use the notation on the left hand side, and the above order is implicitly understood.

To construct the correspondence, we define mappings

$$
\mathcal{F}_{d_{1}, \ldots, d_{n}}^{\left(x_{0}\right)}: \bigotimes_{i=1}^{n} \mathrm{M}_{d_{i}} \rightarrow \mathcal{C}^{\infty}\left(\mathfrak{X}_{n}^{\left(x_{0}\right)}\right)
$$

from the tensor products (4.1) to smooth functions on the restricted chambers 1.5 . To simplify the notation, when the dimensions $d_{1}, \ldots, d_{n}$ are clear from the context, we usually omit the subscripts and write simply $\mathcal{F}^{\left(x_{0}\right)}=\mathcal{F}_{d_{1}, \ldots, d_{n}}^{\left(x_{0}\right)}$. In the representation $\mathrm{M}_{d_{i}}$, let $e_{l_{i}}$ denote the basis vector obtained by applying the generator $F$ repeatedly $l_{i}$ times to the highest weight vector $e_{0}$, as in Section 2.2.2. The mapping 4.2 is defined by setting the images of the tensor product basis vectors to be the functions defined in Section 3.3

$$
\left(\mathcal{F}^{\left(x_{0}\right)}\left[e_{l_{n}} \otimes \cdots \otimes e_{l_{1}}\right]\right)\left(x_{1}, \ldots, x_{n}\right)=\varphi_{l_{1}, \ldots, l_{n}}^{\left(x_{0}\right)}\left(x_{1}, \ldots, x_{n}\right),
$$

and extending linearly.

4.2. Auxiliary formula for two points. The following result will be used both for the proofs of asymptotics in Section 4.3 , and for the anchor point independence, Proposition 4.5. In fact, this result is literally a special case of the latter.

Lemma 4.1. Let $\tau_{0}^{\left(d ; d_{1}, d_{2}\right)} \in \mathrm{M}_{d_{2}} \otimes \mathrm{M}_{d_{1}}$ be as in 2.8), with $d=d_{1}+d_{2}-1-2 m$. Then we have

$$
\left(\mathcal{F}^{\left(x_{0}\right)}\left[\tau_{0}^{\left(d ; d_{1}, d_{2}\right)}\right]\right)\left(x_{1}, x_{2}\right)=q^{\left(\begin{array}{c}
m \\
2
\end{array}\right)} \frac{1}{[m] !} \times \widetilde{\rho}_{0, m}^{\left(x_{0}\right)}\left(x_{1}, x_{2}\right)=\rho_{0, m}^{\left(x_{0}\right)}\left(x_{1}, x_{2}\right) .
$$

More generally, whenever $v \in \mathrm{M}_{d_{2}} \otimes \mathrm{M}_{d_{1}}$ is such that $E . v=0$, then we have

$$
\left(\mathcal{F}^{\left(x_{0}\right)}[v]\right)\left(x_{1}, x_{2}\right)=\sum_{m} c_{m} \times \widetilde{\rho}_{0, m}^{\left(x_{0}\right)}\left(x_{1}, x_{2}\right)
$$

for some coefficients $c_{m} \in \mathbb{C}$ and, in particular, $\left(\mathcal{F}^{\left(x_{0}\right)}[v]\right)\left(x_{1}, x_{2}\right)$ is then independent of $x_{0}$.

Proof. Equation (2.8) gives

$$
v=\tau_{0}^{\left(d ; d_{1}, d_{2}\right)}=\sum_{k=0}^{m} T_{0 ; m}^{k, m-k} \times\left(e_{m-k} \otimes e_{k}\right),
$$

where $T_{0 ; m}^{k, m-k}$ are given by (2.7). Correspondingly, we have

$$
\left(\mathcal{F}^{\left(x_{0}\right)}\left[\tau_{0}^{\left(d ; d_{1}, d_{2}\right)}\right]\right)\left(x_{1}, x_{2}\right)=\sum_{k=0}^{m} T_{0 ; m}^{k, m-k} \times \varphi_{k, m-k}^{\left(x_{0}\right)}\left(x_{1}, x_{2}\right) .
$$

We use Lemma 3.6 to rewrite $\varphi_{k, m-k}^{\left(x_{0}\right)}$ as a linear combination of $\widetilde{\rho}_{k+t, m-k-t}^{\left(x_{0}\right)}$, for $t=0,1, \ldots, m-k$. After straightforward simplifications and a change of the summation index $k$ to $u=k+t$, we obtain

$$
\mathcal{F}^{\left(x_{0}\right)}\left[\tau_{0}^{\left(d ; d_{1}, d_{2}\right)}\right]=\sum_{u=0}^{m} \widetilde{\rho}_{u, m-u}^{\left(x_{0}\right)} \times\left((-1)^{u} q^{\left(\begin{array}{c}
m \\
2
\end{array}\right)+u\left(d_{1}-m\right)} \frac{1}{[m-u] !} \sum_{t=0}^{u}(-1)^{-t} q^{t(u-1)} \frac{1}{[t] ![u-t] !}\right) .
$$


The above sum over $t$ simplifies by Lemma 2.1(d) to

$$
\frac{1}{[u] !} \sum_{t=0}^{u}(-1)^{t} q^{t(u-1)}\left[\begin{array}{c}
u \\
t
\end{array}\right]=\frac{1}{[u] !} q^{\frac{1}{2} u(u-1)} \prod_{s=0}^{u-1}\left(q^{-s}-q^{s}\right)=\left\{\begin{array}{ll}
1 & \text { if } u=0 \\
0 & \text { if } u>0
\end{array} .\right.
$$

Thus finally, for $d=d_{1}+d_{2}-1-2 m$, we obtain the expression

$$
\left(\mathcal{F}^{\left(x_{0}\right)}\left[\tau_{0}^{\left(d ; d_{1}, d_{2}\right)}\right]\right)\left(x_{1}, x_{2}\right)=q^{\left(\begin{array}{c}
m \\
2
\end{array}\right)} \frac{1}{[m] !} \times \widetilde{\rho}_{0, m}^{\left(x_{0}\right)}\left(x_{1}, x_{2}\right) .
$$

This and Lemma 3.2 give the first asserted formula (4.4). By Lemma 2.4 and Remark 2.5, the vectors $v \in \mathrm{M}_{d_{2}} \otimes \mathrm{M}_{d_{1}}$ that satisfy $E . v=0$ are linear combinations of the vectors $\tau_{0}^{\left(d ; d_{1}, d_{2}\right)}$, so the second statement follows.

4.3. Asymptotics via projections to subrepresentations. The detailed understanding of the asymptotics of functions is fundamentally important in applications, since it pertains to the boundary conditions of solutions. Our method describes the asymptotics conceptually with the underlying quantum group in terms of projections to subrepresentations.

The asymptotics are easiest for the functions $\alpha_{l_{1}, \ldots, l_{j-1} ; 0, m ; l_{j+2}, \ldots, l_{n}}^{\left(x_{0}\right)}$ of Section 3.4. Here it is therefore desirable to write the function $\mathcal{F}[v](\boldsymbol{x})$ in terms of these functions. We begin with the following particular case.

Lemma 4.2. Let $\tau_{0}^{\left(d ; d_{j}, d_{j+1}\right)} \in \mathrm{M}_{d_{j+1}} \otimes \mathrm{M}_{d_{j}}$ be as in Lemma 2.4. with $d=d_{j}+d_{j+1}-1-2 m$, and suppose that

$$
v=e_{l_{n}} \otimes \cdots \otimes e_{l_{j+2}} \otimes \tau_{0}^{\left(d ; d_{j}, d_{j+1}\right)} \otimes e_{l_{j-1}} \otimes \cdots \otimes e_{l_{1}}
$$

Then we have

$$
\mathcal{F}^{\left(x_{0}\right)}[v](\boldsymbol{x})=\alpha_{l_{1}, \ldots, l_{j-1} ; 0, m ; l_{j+2}, \ldots, l_{n}}^{\left(x_{0}\right)}(\boldsymbol{x}) .
$$

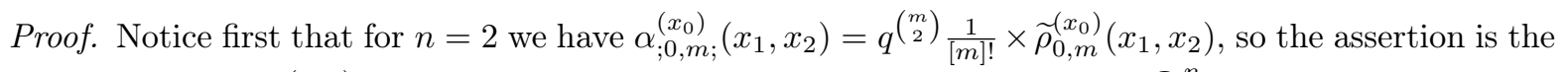
same as Equation (4.4). The general case is similar. Indeed, the vector $v \in \bigotimes_{i=1}^{n} \mathrm{M}_{d_{i}}$ can be expanded in the standard tensor product basis as

$$
v=\sum_{k=0}^{m} T_{0 ; m}^{k, m-k}\left(d_{j}, d_{j+1}\right) \times\left(e_{l_{n}}^{\left(d_{n}\right)} \otimes \cdots \otimes e_{l_{j+2}}^{\left(d_{j+2}\right)} \otimes e_{m-k}^{\left(d_{j+1}\right)} \otimes e_{k}^{\left(d_{j}\right)} \otimes e_{l_{j-1}}^{\left(d_{j-1}\right)} \otimes \cdots \otimes e_{l_{1}}^{\left(d_{1}\right)}\right),
$$

according to Lemma 2.4. Equations (2.8) and (2.7). Correspondingly, we obtain

$$
\mathcal{F}^{\left(x_{0}\right)}[v](\boldsymbol{x})=\sum_{k=0}^{m} T_{0 ; m}^{k, m-k}\left(d_{j}, d_{j+1}\right) \times \varphi_{l_{1}, \ldots, l_{j-1}, k, m-k, l_{j+2}, \ldots, l_{n}}^{\left(x_{0}\right)}(\boldsymbol{x}) .
$$

The terms in this sum are all integrals of the same integrand (up to phase factors). As for the integration contours, the number of loops around any $x_{i}$ is fixed, with the exceptions of $i=j$ and $i=j+1$. The loops around $x_{j}$ and $x_{j+1}$ can be combined by essentially the same calculations that were done in the proofs of Lemmas 3.6 and 4.1 with the asserted result.

With the above particular case established, we now proceed to the following generalization.

Lemma 4.3. Denote $\tau_{\ell}=F^{\ell} \cdot \tau_{0}$. If

$$
v=e_{l_{n}} \otimes \cdots \otimes e_{l_{j+2}} \otimes \tau_{l}^{\left(d ; d_{j}, d_{j+1}\right)} \otimes e_{l_{j-1}} \otimes \cdots \otimes e_{l_{1}},
$$

then (with $d=d_{j}+d_{j+1}-1-2 m$ )

$$
\mathcal{F}^{\left(x_{0}\right)}[v](\boldsymbol{x})=\alpha_{l_{1}, \ldots, l_{j-1} ; l, m ; l_{j+2}, \ldots, l_{n}}^{\left(x_{0}\right)}(\boldsymbol{x}) .
$$


Proof. By definition of the correspondence, we have

$$
\mathcal{F}^{\left(x_{0}\right)}[v](\boldsymbol{x})=\sum_{l_{j}, l_{j+1}} T_{l ; m}^{l_{j}, l_{j+1}}\left(d_{j}, d_{j+1}\right) \times \varphi_{l_{1}, \ldots, l_{n}}^{\left(x_{0}\right)}(\boldsymbol{x}),
$$

with the coefficients $T_{l ; m}^{l_{j}, l_{j+1}}\left(d_{j}, d_{j+1}\right)$ given by Equation 2.10 for $\tau_{l}^{\left(d ; d_{j}, d_{j+1}\right)}=F^{l} \cdot \tau_{0}^{\left(d ; d_{j}, d_{j+1}\right)}$. Therefore, our goal is to rewrite, for any $l \geq 0$,

$$
\alpha_{l_{1}, \ldots, l_{j-1} ; l, m ; l_{j+2}, \ldots, l_{n}}^{\left(x_{0}\right)}(\boldsymbol{x})=\sum_{l_{j}, l_{j+1}} T_{l ; m}^{l_{j}, l_{j+1}}\left(d_{j}, d_{j+1}\right) \times \varphi_{l_{1}, \ldots, l_{n}}^{\left(x_{0}\right)}(\boldsymbol{x}),
$$

with the same coefficients. To achieve this, we will proceed by recursion on $l$. The base case $l=0$ was the content of Lemma 4.2. Using the formula (2.6) for the coproduct $\Delta(F)$, we see that the coefficients 2.10 ) satisfy the recursion

$$
T_{l ; m}^{l_{j}, l_{j+1}}=q^{1-d_{j+1}+2 l_{j+1}} T_{l-1 ; m}^{l_{j}-1, l_{j+1}}+T_{l-1 ; m}^{l_{j}, l_{j+1}-1} .
$$

Hence, it suffices to show the same recursion for the coefficients appearing in Equation (4.5). For that, note that the difference between the integrations defining $\alpha_{\ldots ; l-1, m ; \ldots}^{\left(x_{0}\right)}(\boldsymbol{x})$ and $\alpha_{\ldots ; l, m ; \ldots}^{\left(x_{0}\right)}(\boldsymbol{x})$ is that the latter has one extra integration variable, integrated along a loop that surrounds both $x_{j}$ and $x_{j+1}$ and their associated $w$-variables. Assume (4.5) for the former, and decompose the extra loop of the latter to two pieces, loops anchored at $x_{0}$ that surround $x_{j}$ and $x_{j+1}$ separately. The desired recursion follows by comparing the phase factors after the decomposition.

Fix an index $j \in\{1, \ldots, n-1\}$. Recall Lemma 2.4 especially the decomposition 2.9p of a tensor product to subrepresentations $\mathrm{M}_{d_{j+1}} \otimes \mathrm{M}_{d_{j}} \cong \bigoplus_{d} \mathrm{M}_{d}$. For any $d$ appearing in this sum, the linear map

$$
\begin{aligned}
& \iota_{j, j+1}^{(d)}:\left(\bigotimes_{i=j+2}^{n} \mathrm{M}_{d_{i}}\right) \otimes \mathrm{M}_{d} \otimes \\
& \begin{aligned}
\iota_{j, j+1}^{(d)}\left(e_{l_{n}} \otimes \cdots \otimes e_{l_{j+2}} \otimes e_{l}\right. & \left.\otimes e_{l_{j-1}} \otimes \cdots \otimes \mathrm{M}_{d_{i}}\right) \rightarrow \bigotimes_{i=1}^{n} \mathrm{M}_{d_{i}} \\
& =e_{l_{n}} \otimes \cdots \otimes e_{l_{j+2}} \otimes \tau_{l}^{\left(d ; d_{j}, d_{j+1}\right)} \otimes e_{l_{j-1}} \otimes \cdots \otimes e_{l_{1}}
\end{aligned}
\end{aligned}
$$

is an embedding that respects the action of $\mathcal{U}_{q}\left(\mathfrak{s l}_{2}\right)$. Hence we may interpret the shorter tensor product as a subrepresentation,

$$
\left(\bigotimes_{i=j+2}^{n} \mathrm{M}_{d_{i}}\right) \otimes \mathrm{M}_{d} \otimes\left(\bigotimes_{i=1}^{j-1} \mathrm{M}_{d_{i}}\right) \subset \bigotimes_{i=1}^{n} \mathrm{M}_{d_{i}}
$$

We denote the projection to this subrepresentation by

$$
\pi_{j, j+1}^{(d)}: \bigotimes_{i=1}^{n} \mathrm{M}_{d_{i}} \rightarrow \bigotimes_{i=1}^{n} \mathrm{M}_{d_{i}}
$$

and we denote by

$$
\hat{\pi}_{j, j+1}^{(d)}: \bigotimes_{i=1}^{n} \mathrm{M}_{d_{i}} \rightarrow\left(\bigotimes_{i=j+2}^{n} \mathrm{M}_{d_{i}}\right) \otimes \mathrm{M}_{d} \otimes\left(\bigotimes_{i=1}^{j-1} \mathrm{M}_{d_{i}}\right)
$$

the projection combined with the identification 4.7), so that $\pi_{j, j+1}^{(d)}=\iota_{j, j+1}^{(d)} \circ \hat{\pi}_{j, j+1}^{(d)}$. A vector $v \in$ $\bigotimes_{i=1}^{n} \mathrm{M}_{d_{i}}$ lies in this subrepresentation if and only if $\pi_{j, j+1}^{(d)}(v)=v$, and in this case we typically denote $\hat{v}=\hat{\pi}_{j, j+1}^{(d)}(v)$.

We are now ready to write down the asymptotics of the functions as two consecutive variables tend to a common limit. By the following proposition, the asymptotics are determined by the above decompositions to subrepresentations. 
Proposition 4.4. If $v \in \mathrm{M}_{d_{n}} \otimes \cdots \otimes \mathrm{M}_{d_{1}}$ satisfies $\pi_{j, j+1}^{(d)}(v)=v$, and we denote

$$
\hat{v}=\hat{\pi}_{j, j+1}^{(d)}(v) \in\left(\bigotimes_{i=j+2}^{n} \mathrm{M}_{d_{i}}\right) \otimes \mathrm{M}_{d} \otimes\left(\bigotimes_{i=1}^{j-1} \mathrm{M}_{d_{i}}\right),
$$

then we have the asymptotics

$$
\begin{aligned}
& \lim _{x_{j}, x_{j+1} \rightarrow \xi}\left(\left(x_{j+1}-x_{j}\right)^{-\Delta_{d}^{d_{j}, d_{j+1}}} \times \mathcal{F}_{d_{1}, \ldots, d_{n}}^{\left(x_{0}\right)}[v]\left(x_{1}, \ldots, x_{n}\right)\right) \\
& =B_{d}^{d_{j}, d_{j+1}} \times \mathcal{F}_{d_{1}, \ldots, d_{j-1}, d, d_{j+2}, \ldots, d_{n}}^{\left(x_{0}\right)}[\hat{v}]\left(x_{1}, \ldots, x_{j-1}, \xi, x_{j+2}, \ldots, x_{n}\right),
\end{aligned}
$$

where the exponent $\Delta_{d}^{d_{j}, d_{j+1}}$ is given by (3.7) and the multiplicative constant $B_{d}^{d_{j}, d_{j+1}}$ by 3.8 .

Proof. The vector $v$ can be expressed as a linear combination of $e_{l_{n}} \otimes \cdots \otimes e_{l_{j+2}} \otimes \tau_{l}^{\left(d ; d_{j}, d_{j+1}\right)} \otimes e_{l_{j-1}} \otimes$ $\cdots \otimes e_{l_{1}}$. For these vectors, the assertion follows by combining Lemmas 4.3 and 3.11 .

4.4. Anchor point independence. Next we show that for highest weight vectors, the corresponding functions become well-defined on the chamber $\mathfrak{X}_{n}$ of $(1.2)$. This is the simplest manifestation of the closedness of the corresponding integration surface.

Proposition 4.5. If $v \in \mathrm{M}_{d_{n}} \otimes \cdots \otimes \mathrm{M}_{d_{1}}$ is such that E. $v=0$, then we have

$$
\left(\mathcal{F}^{\left(x_{0}\right)}[v]\right)(\boldsymbol{x})=\sum_{m_{2}, m_{3}, \ldots, m_{n}} c_{m_{2}, \ldots, m_{n}} \times \widetilde{\rho}_{0, m_{2}, \ldots, m_{n}}^{\left(x_{0}\right)}(\boldsymbol{x})
$$

for some coefficients $c_{m_{2}, \ldots, m_{n}} \in \mathbb{C}$. In particular $\left(\mathcal{F}^{\left(x_{0}\right)}[v]\right)(\boldsymbol{x})$ is independent of $x_{0}$.

Proof. As a warm up, observe that in the case $n=1$ the statement is immediate: by Lemma 3.5 the basis vectors $e_{l_{1}} \in \mathrm{M}_{d_{1}}$ are mapped to $\mathcal{F}^{\left(x_{0}\right)}\left[e_{l_{1}}\right] \propto \widetilde{\rho}_{l_{1}}^{\left(x_{0}\right)}$, and we have E. $e_{l_{1}}=0$ only if $l_{1}=0$. For the case $n=2$ the statement was already shown in Lemma 4.1 .

We proceed by induction on $n$. Let $n>2$ and write $v$ in the basis $e_{l_{n}} \otimes \cdots \otimes e_{l_{3}} \otimes \tau_{l}^{(d)}$, with $l, l_{3}, \ldots, l_{n}$ non-negative integers and $d=d_{1}+d_{2}-2 m$ for some $m$ as in Lemma 2.4 .

$$
v=\sum_{l, d, l_{3}, \ldots, l_{n}} b_{l, d ; l_{3}, \ldots, l_{n}} \times e_{l_{n}} \otimes \cdots \otimes e_{l_{3}} \otimes \tau_{l}^{(d)} .
$$

Separate the parts corresponding to fixed values of $d$, and denote (no summation over $d$ here)

$$
\begin{array}{r}
v^{(d)}=\sum_{l, l_{3}, \ldots, l_{n}} b_{l, d ; l_{3}, \ldots, l_{n}} \times e_{l_{n}} \otimes \cdots \otimes e_{l_{3}} \otimes \tau_{l}^{(d)} \in \bigotimes_{i=1}^{n} \mathrm{M}_{d_{i}} \quad \text { and } \\
\pi_{1,2}^{(d)}\left(v^{(d)}\right)=\hat{v}^{(d)}=\sum_{l, l_{3}, \ldots, l_{n}} b_{l, d ; l_{3}, \ldots, l_{n}} \times e_{l_{n}} \otimes \cdots \otimes e_{l_{3}} \otimes e_{l} \in\left(\bigotimes_{i=3}^{n} \mathrm{M}_{d_{i}}\right) \otimes \mathrm{M}_{d} .
\end{array}
$$

Now $\hat{v}^{(d)}$ also satisfies $E \cdot \hat{v}^{(d)}=0$, and the tensor product only has $n-1$ tensorands. By the induction hypothesis,

$$
\left(\mathcal{F}^{\left(x_{0}\right)}\left[\hat{v}^{(d)}\right]\right)\left(\xi, x_{3}, \ldots, x_{n}\right)=\sum_{m_{3}, \ldots, m_{n}} \hat{c}_{m_{3}, \ldots, m_{n}}^{(d)} \times \widetilde{\rho}_{0, m_{3}, \ldots, m_{n}}^{\left(x_{0}\right)}\left(\xi, x_{3}, \ldots, x_{n}\right),
$$

where the implicit dimension parameters are $d, d_{3}, d_{4}, \ldots, d_{n}$ now. In view of Lemma 4.3 and the rearrangement procedure of integration contours similar to the proofs of Lemmas 3.7 and 3.6 , one sees that also

$$
\left(\mathcal{F}^{\left(x_{0}\right)}\left[v^{(d)}\right]\right)\left(x_{1}, x_{2}, x_{3}, \ldots, x_{n}\right)=\sum_{m_{3}, \ldots, m_{n}} c_{m_{3}, \ldots, m_{n}}^{(d)} \times \widetilde{\rho}_{0, m_{2}, \ldots, m_{n}}^{\left(x_{0}\right)}\left(x_{1}, x_{2}, x_{3}, \ldots, x_{n}\right) .
$$

The conclusion is finally obtained by summing over $d$, since $v=\sum_{d} v^{(d)}$. 
Remark 4.6. When $\kappa$ is large enough, (3.4), the integrals $\widetilde{\rho}_{m_{1}, \ldots, m_{n}}^{\left(x_{0}\right)}$ are convergent. When E. $v=0$, the above proposition then shows that $\mathcal{F}^{\left(x_{0}\right)}[v]\left(x_{1}, \ldots, x_{n}\right)$ is independent of $x_{0}$. By analyticity in $\kappa$, this independence of $x_{0}$ holds for all $\kappa$, and we get a well-defined function $\mathcal{F}[v]: \mathfrak{X}_{n} \rightarrow \mathbb{C}$ by

$$
\mathcal{F}[v]\left(x_{1}, \ldots, x_{n}\right)=\mathcal{F}^{\left(x_{0}\right)}[v]\left(x_{1}, \ldots, x_{n}\right) \quad \text { for any } \quad x_{0}<x_{1} .
$$

4.5. Integration by parts formula. According to the definition given in Section 4.1 the vectors $v \in \mathrm{M}_{d_{n}} \otimes \cdots \otimes \mathrm{M}_{d_{1}}$ determine linear combinations of integration surfaces. With a suitable interpretation, there is a homology theory for these, in which the boundary operator corresponds to the action of the quantum group generator E, see [FW91]. We will only make use of a version of Stokes formula, i.e., integration by parts, which we state and prove next.

Lemma 4.7. Let $l_{1}, \ldots, l_{n} \in \mathbb{Z}_{\geq 0}$ and $\ell=\sum_{j} l_{j}$. Suppose that $g\left(w_{\ell} ; \boldsymbol{x} ; w_{1}, \ldots, w_{\ell-1}\right)$ is a holomorphic function of the $\ell$ variables $w_{1}, \ldots, w_{\ell}$ defined on $\mathfrak{W}^{(\ell)}$, which is symmetric in the $\ell-1$ variables $w_{1}, \ldots, w_{\ell-1}$. Then we have

$$
\begin{aligned}
& \int_{\mathfrak{L}_{l_{1}}^{\ni}, \ldots, l_{n}} \sum_{r=1}^{\ell} \frac{\partial}{\partial w_{r}}\left(g\left(w_{r} ; \boldsymbol{x} ; w_{1}, \ldots, w_{r-1}, w_{r+1}, \ldots, w_{\ell}\right) f_{l_{1}, \ldots, l_{n}}^{\ni}(\boldsymbol{x} ; \boldsymbol{w})\right) \mathrm{d} w_{1} \cdots \mathrm{d} w_{\ell} \\
= & \sum_{j=1}^{n}\left\{\left(q^{-1}-q\right)\left[l_{j}\right]\left[d_{j}-l_{j}\right] q^{\sum_{i<j}\left(d_{i}-1-2 l_{i}\right)}\right. \\
& \left.\times \int_{\mathfrak{L}_{l_{1}, \ldots, l_{j}-1, \ldots, l_{n}}^{\ni}}\left(\gamma\left(\boldsymbol{x} ; w_{1}, \ldots, w_{\ell-1}\right) f_{l_{1}, \ldots, l_{j}-1, \ldots, l_{n}}^{\ni}\left(\boldsymbol{x} ; w_{1}, \ldots, w_{\ell-1}\right)\right) \mathrm{d} w_{1} \cdots \mathrm{d} w_{\ell-1}\right\},
\end{aligned}
$$

where

$$
\gamma\left(\boldsymbol{x} ; w_{1}, \ldots, w_{\ell-1}\right)=\prod_{i=1}^{n}\left|x_{0}-x_{i}\right|^{-\frac{4}{\kappa}\left(d_{i}-1\right)} \prod_{s \neq r}\left|x_{0}-w_{r}\right|^{\frac{8}{\kappa}} g\left(x_{0} ; \boldsymbol{x} ; w_{1}, \ldots, w_{\ell-1}\right) .
$$

Proof. Let us first perform an integration by parts in a single term in 4.8. Fix $r$, and let $x_{j}$ be the point encircled by the loop of $w_{r}$ in $\mathfrak{L}_{l_{1}, \ldots, l_{n}}^{\ni}$, and denote by $u$ the number of $s<r$ such that $w_{s}$ also encircles the same point $x_{j}$, that is, $u=r-1-\sum_{i<j} l_{i}$. We then perform integration by parts in the integral over $w_{r}$, and notice that the boundary terms from the beginning and end points of the loop only differ by a phase (formally $w_{r}=x_{0}$ both at the beginning and the end, but on different sheets of a Riemann surface). After this integration by parts, the contribution of the $r$ :th term becomes

$$
\begin{aligned}
& q^{\sum_{i<j}\left(d_{i}-1-2 l_{i}\right)}\left(q^{1-d_{j}+2 u}-q^{-1+d_{j}-2 u}\right) \\
& \quad \times \int_{\mathfrak{S}_{l_{1}, \ldots, l_{j}-1, \ldots, l_{n}}^{\ni}}\left(\gamma\left(\boldsymbol{x} ; w_{1}, \ldots, w_{\ell-1}\right) f_{l_{1}, \ldots, l_{j}-1, \ldots, l_{n}}^{\ni}\left(\boldsymbol{x} ; w_{1}, \ldots, w_{\ell-1}\right)\right) \mathrm{d} w_{1} \cdots \mathrm{d} w_{\ell-1},
\end{aligned}
$$

where we relabeled the other integration variables and used the assumption of symmetric dependence of $g$ on them (and a similar property of $f$ ). We collect the terms corresponding to the same $j$, and use Lemma 2.1(a) in the form

$$
\sum_{u=0}^{l_{j}-1}\left(q^{1-d_{j}+2 u}-q^{-1+d_{j}-2 u}\right)=\left(q^{-1}-q\right)\left[l_{j}\right]\left[d_{j}-l_{j}\right]
$$

to simplify the sum of these terms. This concludes the proof.

Besides the anchor point independence of Proposition 4.5, the closedness of the integration surface for highest weight vectors is used in combination with Stokes' formula via the following corollary. 
Corollary 4.8. Let $\ell \in \mathbb{Z}_{\geq 0}$. Suppose that $g\left(w_{\ell} ; \boldsymbol{x} ; w_{1}, \ldots, w_{\ell-1}\right)$ is a holomorphic function of the $\ell$ variables $w_{1}, \ldots, w_{\ell}$ defined on $\mathfrak{W}^{(\ell)}$, which is symmetric in the $\ell-1$ variables $w_{1}, \ldots, w_{\ell-1}$. If

$$
v=\sum_{\substack{l_{1}, \ldots, l_{n} \geq 0 \\ l_{1}+\cdots+l_{n}=\ell}} t_{l_{1}, \ldots, l_{n}} e_{l_{n}} \otimes \cdots \otimes e_{l_{1}}
$$

satisfies E.v $=0$, then

$$
\sum_{l_{1}, \ldots, l_{n}} t_{l_{1}, \ldots, l_{n}} \int_{\mathfrak{L}_{l_{1}, \ldots, l_{n}}^{\ni}} \sum_{r=1}^{\ell} \frac{\partial}{\partial w_{r}}\left(g\left(w_{r} ; \boldsymbol{x} ; w_{1}, \ldots, w_{r-1}, w_{r+1}, \ldots, w_{\ell}\right) f_{l_{1}, \ldots, l_{n}}^{\ni}(\boldsymbol{x} ; \boldsymbol{w})\right) \mathrm{d} w_{1} \cdots \mathrm{d} w_{\ell}=0 .
$$

Proof. On the left hand side of the asserted formula, we use the previous lemma in each term, and write the left hand side as

$$
\left(q^{-1}-q\right) \sum_{j=1}^{n} \sum_{l_{1}, \ldots, l_{n}}\left[l_{j}\right]\left[d_{j}-l_{j}\right] q^{\sum_{i<j}\left(d_{i}-1-2 l_{i}\right)} t_{l_{1}, \ldots, l_{n}} \times\left(\int_{\mathfrak{L}_{l_{1}, \ldots, l_{j}-1, \ldots, l_{n}}} \gamma f_{l_{1}, \ldots, l_{j}-1, \ldots, l_{n}}^{\ni}\right) .
$$

On the other hand, using Lemma 2.2 for the iterated coproduct $\Delta^{(n)}(E)$, we can expand E.v in the tensor product basis as

$$
\sum_{j=1}^{n} \sum_{l_{1}, \ldots, l_{n}}\left[l_{j}\right]\left[d_{j}-l_{j}\right] q^{\sum_{i<j}\left(d_{i}-1-2 l_{i}\right)} t_{l_{1}, \ldots, l_{n}} \times e_{l_{n}} \otimes \cdots \otimes e_{l_{j}-1} \otimes \cdots \otimes e_{l_{1}} .
$$

Apart from the factor $\left(q^{-1}-q\right)$, the coefficient of $e_{l_{n}} \otimes \cdots \otimes e_{l_{j}-1} \otimes \cdots \otimes e_{l_{1}}$ is the same as the coefficient of the integral over $\mathfrak{L}_{l_{1}, \ldots, l_{j}-1, \ldots, l_{n}}^{\ni}$ in 4.9 . Therefore, by the assumption $E . v=0$, the expression 4.9 vanishes.

4.6. Partial differential equations. Now we turn to partial differential equations satisfied by the functions associated to highest weight vectors.

Define, for any $j=1, \ldots, n$ and $p \in \mathbb{Z}$, the first order partial differential operators

$$
\mathcal{L}_{-p}^{(j)}:=-\sum_{i \neq j}\left(\left(x_{i}-x_{j}\right)^{1-p} \frac{\partial}{\partial x_{i}}+(1-p) h_{1, d_{i}}\left(x_{i}-x_{j}\right)^{-p}\right),
$$

where $h_{1, d}$ is given by Equation 1.1. Define also, for any $j=1, \ldots, n$, a partial differential operator of order $d_{j}$ by the Benoit \& Saint-Aubin formula BSA88.

$$
\mathcal{D}_{d_{j}}^{(j)}:=\sum_{k=1}^{d_{j}} \sum_{\substack{p_{1}, \ldots, p_{k} \geq 1 \\ p_{1}+\cdots+p_{k}=d_{j}}} \frac{(-4 / \kappa)^{d_{j}-k}\left(d_{j}-1\right) !^{2}}{\prod_{u=1}^{k-1}\left(\sum_{i=1}^{u} p_{i}\right)\left(\sum_{i=u+1}^{k} p_{i}\right)} \times \mathcal{L}_{-p_{1}}^{(j)} \cdots \mathcal{L}_{-p_{k}}^{(j)} .
$$

The following special case without any integrations will be used to verify the PDEs in the general case.

Lemma 4.9. Let $d_{1}, \ldots, d_{n}$ be real numbers. The function

$$
f^{(0)}\left(x_{1}, \ldots, x_{n}\right)=\prod_{1 \leq i<j \leq n}\left(x_{j}-x_{i}\right)^{\frac{2}{\kappa}\left(d_{i}-1\right)\left(d_{j}-1\right)}
$$

satisfies the partial differential equation $\mathcal{D}_{d_{j}}^{(j)} f^{(0)}=0$, for all $j=1, \ldots, n$ such that $d_{j}$ is a positive integer.

We postpone the proof to Appendix B. 
Remark 4.10. The function $f^{(0)}$ is a very simple product of powers of differences, but it is nevertheless not entirely trivial to verify that the Benoit $\&$ Saint-Aubin differential operators $\mathcal{D}_{d_{j}}^{(j)}$ annihilate it in general. The proof that we present in Appendix B requires only performing the explicit calculation in the case of $d_{j}=2$, and then using recursively a fusion argument of the type considered in Dub15, Theorem 15]. There is one stage in this argument, [Dub15, Lemma 1], which appeals to the structure of Verma modules for the Virasoro algebra, but only in a simple case when $\kappa \notin \mathbb{Q}$.

As an alternative proof for Lemma 4.9, which is shorter but appeals to more involved constructions, one could use properties of vertex operators and the BRST charge, see e.g. [Fel89].

The interpretation of the following corollary is that the Benoit \& Saint-Aubin differential operators acting on our integrand produce exact forms.

Corollary 4.11. The function $f^{(\ell)}$ satisfies, for any $j=1, \ldots, n$,

$$
\left(\mathcal{D}_{d_{j}}^{(j)} f^{(\ell)}\right)(\boldsymbol{x} ; \boldsymbol{w})=\sum_{r=1}^{\ell} \frac{\partial}{\partial w_{r}}\left(g\left(w_{r} ; \boldsymbol{x} ; w_{1}, \ldots, w_{r-1}, w_{r+1}, \ldots, w_{\ell}\right) \times f^{(\ell)}(\boldsymbol{x} ; \boldsymbol{w})\right),
$$

where $g$ is a rational function which is symmetric in the last $\ell-1$ variables, and whose only poles are where some of its arguments coincide.

Proof. Denote $\hat{n}=n+\ell$ and

$$
\hat{\boldsymbol{x}}=\left(x_{1}, \ldots, x_{n}, w_{1}, \ldots, w_{\ell}\right)
$$

and define the function, a product of powers of differences of variables,

$$
\hat{f}^{(0)}(\hat{\boldsymbol{x}})=f^{(\ell)}(\boldsymbol{x} ; \boldsymbol{w}) .
$$

The conformal weights $h$ associated to the variables $w_{r}$ are $h=h_{1,-1}=1$. We will apply Lemma 4.9 to the function $\hat{f}^{(0)}$ of $\hat{n}$ variables. We keep the notation $\mathcal{L}_{p}^{(j)}$ and $\mathcal{D}_{d_{j}}^{(j)}$ for the differential operators in the $n$ variables $x_{1}, \ldots, x_{n}$, and use the notation $\hat{\mathcal{L}}_{p}^{(j)}$ and $\hat{\mathcal{D}}_{d_{j}}^{(j)}$ for the differential operators in $\hat{n}=n+\ell$ variables, that are appropriate for the application of the previous lemma. Explicitly, we have

$$
\hat{\mathcal{L}}_{p}^{(j)}=\mathcal{L}_{p}^{(j)}-\sum_{r=1}^{\ell} D_{w_{r}} M_{\left(w_{r}-x_{j}\right)^{1+p}}
$$

where $D_{w_{r}}$ is the differential operator $\frac{\partial}{\partial w_{r}}$, and $M_{\left(w_{r}-x_{j}\right)^{1+p}}$ is the multiplication operator by the function $\left(w_{r}-x_{j}\right)^{1+p}$, and we used the fact that, since $h_{1,-1}=1$,

$$
D_{w_{r}} M_{\left(w_{r}-x_{j}\right)^{1+p}}=\left(w_{r}-x_{j}\right)^{1+p} \frac{\partial}{\partial w_{r}}+(1+p) h_{1,-1}\left(w_{r}-x_{j}\right)^{p} .
$$

The operator $\hat{\mathcal{D}}_{d_{j}}^{(j)}$ is a linear combination of terms, which we expand by the binomial formula

$$
\hat{\mathcal{L}}_{-n_{1}}^{(j)} \cdots \hat{\mathcal{L}}_{-n_{k}}^{(j)}=\sum_{A \subset\{1, \ldots, k\}} O_{1}^{A} \cdots O_{k}^{A}, \quad \text { where } \quad O_{a}^{A}=\left\{\begin{array}{ll}
\mathcal{L}_{-n_{a}}^{(j)} & \text { if } a \notin A \\
\sum_{r} D_{w_{r}} M_{\left(w_{r}-x_{j}\right)^{1-n_{a}}} & \text { if } a \in A
\end{array} .\right.
$$

The conclusion of Lemma 4.9 reads

$$
0=\left(\hat{\mathcal{D}}_{d_{j}}^{(j)} \hat{f}^{(0)}\right)(\hat{\boldsymbol{x}})=\left(\hat{\mathcal{D}}_{d_{j}}^{(j)} f^{(\ell)}\right)(\boldsymbol{x} ; \boldsymbol{w}) .
$$

Expanding by the binomial formula, we observe that the terms with $A=\emptyset$ give precisely the LHS of the assertion, namely $\mathcal{D}_{d_{j}}^{(j)} f^{(\ell)}$. When $A \neq \emptyset$, choose the minimal $a \in A$, and write the term in the form

$$
\sum_{r} D_{w_{r}} \mathcal{L}_{-n_{1}}^{(j)} \cdots \mathcal{L}_{-n_{a-1}}^{(j)} M_{\left(w_{r}-x_{j}\right)^{1-n_{a}}} O_{a+1}^{A} \cdots O_{k}^{A} f^{(\ell)}
$$


by noticing that $\mathcal{L}_{-n_{1}}^{(j)} \cdots \mathcal{L}_{-n_{a-1}}^{(j)}$ does not contain $w_{r}$ and can be moved inside the differentiation $D_{w_{r}}$. These remaining terms put together constitute the RHS of the assertion

$$
\sum_{r=1}^{\ell} \frac{\partial}{\partial w_{r}}\left(g\left(w_{r} ; \boldsymbol{x} ; w_{1}, \ldots, w_{r-1}, w_{r+1}, \ldots, w_{\ell}\right) \times f^{(\ell)}(\boldsymbol{x} ; \boldsymbol{w})\right) .
$$

It is easy to see that $g$ has no poles where the variables do not coincide, and is symmetric in its last $\ell-1$ variables.

We now conclude by the important property that the functions which correspond to highest weight vectors satisfy the Benoit \& Saint-Aubin partial differential equations.

Proposition 4.12. If E.v $=0$, then the function $\mathcal{F}[v]$ satisfies $\mathcal{D}_{d_{j}}^{(j)} \mathcal{F}[v]=0$.

Proof. By linearity, we may assume that $v$ is an eigenvector of $K$, i.e. that the number of integration variables $\ell$ is fixed. Dominated convergence ensures that we can take the differential operator $\mathcal{D}_{d_{j}}^{(j)}$ inside the integral, and thus let it act directly to the integrand $f^{(\ell)}$. Corollary 4.11 then implies that $\mathcal{D}_{d_{j}}^{(j)} \mathcal{F}[v](\boldsymbol{x})$ is a linear combination of terms of the form studied in the integration by parts formula, Lemma 4.7 Assuming E.v $=0$ it follows from Corollary 4.8 that this linear combination vanishes.

Remark 4.13. If one of the tensorands is a trivial representation, $d_{i}=1$, the $i: t h P D E$ is merely

$$
\frac{\partial}{\partial x_{i}} \mathcal{F}[v]\left(x_{1}, \ldots, x_{i}, \ldots, x_{n}\right)=0
$$

which is also a consequence of the statement of Lemma 3.4. If $d_{i}=2$, the $i$ :th PDE is similar to [1.6], and it can always be interpreted as a local martingale property of the function for a chordal $\mathrm{SLE}_{\kappa}$.

4.7. Möbius covariance. Translation invariance and scaling covariance were shown for all basis functions $\varphi^{\left(x_{0}\right)}$ in Lemma 3.3. We will now show that if a vector $v$ is in a trivial (one-dimensional) subrepresentation of the entire tensor product, then the corresponding function $\mathcal{F}[v]$ transforms covariantly under all Möbius transformations as in (1.4), with covariance weights $h_{1, d_{i}}$ given by (1.1).

We first record a property of the integrand, which will be used in the proof of Möbius covariance.

Lemma 4.14. If $\ell=\frac{1}{2} \sum_{i=1}^{n}\left(d_{i}-1\right)$, then the function $f^{(\ell)}$ satisfies the partial differential equation

$$
\left(\sum_{i=1}^{n}\left(x_{i}^{2} \frac{\partial}{\partial x_{i}}+2 h_{1, d_{i}} x_{i}\right)\right) f^{(\ell)}(\boldsymbol{x} ; \boldsymbol{w})=\sum_{r=1}^{\ell} \frac{\partial}{\partial w_{r}}\left(g\left(w_{r} ; \boldsymbol{x} ; w_{1}, \ldots, w_{r-1}, w_{r+1}, \ldots, w_{\ell}\right) \times f^{(\ell)}(\boldsymbol{x} ; \boldsymbol{w})\right),
$$

where $g$ is a rational function which is symmetric in the last $\ell-1$ variables, and whose only poles are where some of its arguments coincide.

Proof. We will perform an explicit calculation that shows the claimed identity, with

$$
g\left(w_{r} ; \boldsymbol{x} ; w_{1}, \ldots, w_{r-1}, w_{r+1}, \ldots, w_{\ell}\right)=-w_{r}^{2}+\prod_{i=1}^{n}\left(w_{r}-x_{i}\right)^{d_{i}-1} \prod_{s \neq r}\left(w_{r}-w_{s}\right)^{-2} .
$$

One can begin by observing, by a direct calculation that uses the assumption $\ell=\frac{1}{2} \sum_{i=1}^{n}\left(d_{i}-1\right)$, that

$$
\begin{aligned}
& \left(\sum_{i=1}^{n}\left(x_{i}^{2} \frac{\partial}{\partial x_{i}}+2 h_{1, d_{i}} x_{i}\right)\right) f^{(\ell)}(\boldsymbol{x} ; \boldsymbol{w}) \\
= & -\sum_{r=1}^{\ell} \frac{\partial}{\partial w_{r}}\left(w_{r}^{2} f^{(\ell)}(\boldsymbol{x} ; \boldsymbol{w})\right)+\left(1-\frac{4}{\kappa}\right)\left(\sum_{i=1}^{n}\left(1-d_{i}\right) x_{i}+2 \sum_{r=1}^{\ell} w_{r}\right) f^{(\ell)}(\boldsymbol{x} ; \boldsymbol{w}) .
\end{aligned}
$$


Comparing with the explicit $g$ in Equation 4.10, the claim now reduces to the vanishing of

$$
\sum_{r=1}^{\ell} \prod_{i=1}^{n}\left(w_{r}-x_{i}\right)^{d_{i}-1} \prod_{s \neq r}\left(w_{r}-w_{s}\right)^{-2}\left(\sum_{j=1}^{n} \frac{d_{j}-1}{w_{r}-x_{j}}-2 \sum_{u \neq r} \frac{1}{w_{r}-w_{u}}\right)-2 \sum_{r=1}^{\ell} w_{r}+\sum_{i=1}^{n}\left(d_{i}-1\right) x_{i},
$$

which is a rational function in the variables $w_{r}, r=1, \ldots, \ell$. Note that there are no poles of (4.11) except possibly poles of degree at most three at $w_{r}=w_{s}$ for some $r \neq s$. To show that these points are in fact not poles, it is by symmetry sufficient to consider the Laurent series expansion in, for example, the difference $\epsilon=w_{2}-w_{1}$. This can be done in a straightforward manner. In addition, one verifies that with fixed $w_{2}, \ldots, w_{\ell}$, as $w_{1} \rightarrow \infty$, the function tends to zero. Thus, the expression (4.11) is an entire function of $w_{1}$ tending to zero at infinity, and as such vanishes identically. This concludes the proof.

Proposition 4.15. If E.v $=0$ and K.v $=v$, then for any Möbius transformation $\mu: \mathbb{H} \rightarrow \mathbb{H}$ such that $\mu\left(x_{1}\right)<\mu\left(x_{2}\right)<\cdots<\mu\left(x_{n}\right)$, the function $\mathcal{F}[v]$ satisfies

$$
\prod_{i=1}^{n} \mu^{\prime}\left(x_{i}\right)^{h_{1, d_{i}}} \times \mathcal{F}[v]\left(\mu\left(x_{1}\right), \ldots, \mu\left(x_{n}\right)\right)=\mathcal{F}[v]\left(x_{1}, \ldots, x_{n}\right) .
$$

Proof. Any Möbius transformation $\mu: \mathbb{H} \rightarrow \mathbb{H}$ can be written as a composition of a translation $z \mapsto z+\xi$ (for some $\xi \in \mathbb{R}$ ), a scaling $z \mapsto \lambda z$ (for some $\lambda>0$ ), and a special conformal transformation $z \mapsto \frac{z}{1+a z}$ (for some $a \in \mathbb{R}$ ). Lemma 3.3 shows the assertion for translations and scalings. It suffices to prove the statement for special conformal transformations.

For the special conformal transformation, we may assume that $x_{1}<0$ and $x_{n}>0$, by precomposing with a translation if necessary. Then, the special conformal transformation $\mu_{a}(z)=\frac{z}{1+a z}$ respects the order of the boundary points $x_{1}, \ldots, x_{n}$ if $a \in\left(\frac{-1}{x_{n}}, \frac{-1}{x_{1}}\right)$. To obtain the general case we will integrate an infinitesimal form of the formula starting from the trivial case of $a=0$.

Consider a term

$$
\prod_{i=1}^{n} \mu^{\prime}\left(x_{i}\right)^{h_{1, d_{i}}} \times \int_{\mathfrak{L}_{l_{1}, \ldots, l_{n}}} f_{l_{1}, \ldots, l_{n}}^{\ni}\left(\mu_{a}\left(x_{1}\right), \ldots, \mu_{a}\left(x_{n}\right) ; w_{1}, \ldots, w_{\ell}\right) \mathrm{d} w_{1} \cdots \mathrm{d} w_{\ell}
$$

on the left hand side of the asserted equation. Using the identities $\frac{\mathrm{d}}{\mathrm{d} a} \mu_{a}(z)=-\mu_{a}(z)^{2}$ and $\frac{\mathrm{d}}{\mathrm{d} a}\left(\mu_{a}^{\prime}(z)^{h}\right)=$ $-2 h \mu_{a}(z) \times \mu_{a}^{\prime}(z)^{h}$ we compute its derivative with respect to $a$,

$$
\begin{aligned}
& \frac{\mathrm{d}}{\mathrm{d} a}\left(\prod_{i=1}^{n} \mu^{\prime}\left(x_{i}\right)^{h_{1, d_{i}}} \times \int_{\mathfrak{L}_{l_{1}, \ldots, l_{n}}^{\ni}} f_{l_{1}, \ldots, l_{n}}^{\ni}\left(\mu_{a}\left(x_{1}\right), \ldots, \mu_{a}\left(x_{n}\right) ; \boldsymbol{w}\right) \mathrm{d} w_{1} \cdots \mathrm{d} w_{\ell}\right) \\
= & \left(\prod_{i=1}^{n} \mu^{\prime}\left(x_{i}\right)^{h_{1, d_{i}}}\right) \times \int_{\mathfrak{L}_{\mathfrak{L}_{1}, \ldots, l_{n}}^{\ni}}\left(\mathcal{L}_{1} f_{l_{1}, \ldots, l_{n}}^{\ni}\right)\left(\mu_{a}\left(x_{1}\right), \ldots, \mu_{a}\left(x_{n}\right) ; \boldsymbol{w}\right) \mathrm{d} w_{1} \cdots \mathrm{d} w_{\ell},
\end{aligned}
$$

where

$$
\mathcal{L}_{1}=-\sum_{i=1}^{n}\left(x_{i}^{2} \frac{\partial}{\partial x_{i}}+2 h_{1, d_{i}} x_{i}\right)
$$

The assumption $K . v=v$ guarantees that in these terms $\ell=\sum_{i} l_{i}$ takes the value $\ell=\frac{1}{2} \sum_{i=1}^{n}\left(d_{i}-1\right)$. Thus, by Lemma 4.14, we have

$$
\left(\mathcal{L}_{1} f_{l_{1}, \ldots, l_{n}}^{\ni}\right)(\boldsymbol{x} ; \boldsymbol{w})=\sum_{r=1}^{\ell} \frac{\partial}{\partial w_{r}}\left(g\left(w_{r} ; \boldsymbol{x} ; w_{1}, \ldots, w_{r-1}, w_{r+1}, \ldots, w_{\ell}\right) \times f_{l_{1}, \ldots, l_{n}}^{\ni}(\boldsymbol{x} ; \boldsymbol{w})\right),
$$


where $g$ single-valued and symmetric with respect to the last $\ell-1$ variables. Since E.v $=0$ we can apply Corollary 4.8 to the $a$-derivative of the left hand side of the asserted formula, and get

$$
\frac{\mathrm{d}}{\mathrm{d} a}\left(\prod_{i=1}^{n} \mu^{\prime}\left(x_{i}\right)^{h_{1, d_{i}}} \times \mathcal{F}[v]\left(\mu\left(x_{1}\right), \ldots, \mu\left(x_{n}\right)\right)\right)=0 .
$$

It now follows that also the left hand side of the asserted formula is constant in $a$ for $a \in\left(\frac{-1}{x_{n}}, \frac{-1}{x_{1}}\right)$. At $a=0$ we have $\mu_{a}=\operatorname{id}_{\mathbb{H}}$, so this constant equals $\mathcal{F}[v]\left(x_{1}, \ldots, x_{n}\right)$.

4.8. Main theorems about the correspondence. We now give the precise statements of the main results about the spin chain - Coulomb gas correspondence, and finish their proofs. These results show how the properties of the vector $v \in \mathrm{M}_{d_{n}} \otimes \cdots \otimes \mathrm{M}_{d_{1}}$ translate to properties of the function $\left(x_{0} ; x_{1}, \ldots, x_{n}\right) \mapsto \mathcal{F}^{\left(x_{0}\right)}[v]\left(x_{1}, \ldots, x_{n}\right)$. Three types of properties of the functions are considered: partial differential equations (PDE), covariance properties (COV) and asymptotics (ASY).

Recall the following notation, to be used in the statement below. The exponents $h_{1, d}$ and $\Delta_{d}^{d_{1}, \ldots, d_{n}}$ are given by

$$
h_{1, d}=\frac{(d-1)(2(d+1)-\kappa)}{2 \kappa} \quad \text { and } \quad \Delta_{d}^{d_{1}, \ldots, d_{n}}=h_{1, d}-\sum_{i=1}^{n} h_{1, d_{i}} .
$$

The multiplicative constants $B_{d}^{d^{\prime}, d^{\prime \prime}}$ are defined by the analytic continuation in $\kappa$ of the integrals (3.8) over $m$-dimensional simplices, with $m=\frac{1}{2}\left(d^{\prime}+d^{\prime \prime}-1-d\right)$, which by Remark 3.9 take the form

$$
B_{d}^{d^{\prime}, d^{\prime \prime}}=\frac{1}{m !} \prod_{p=1}^{m} \frac{\Gamma\left(1-\frac{4}{\kappa}\left(d^{\prime}-p\right)\right) \Gamma\left(1-\frac{4}{\kappa}\left(d^{\prime \prime}-p\right)\right) \Gamma\left(1+\frac{4}{\kappa} p\right)}{\Gamma\left(1+\frac{4}{\kappa}\right) \Gamma\left(2-\frac{4}{\kappa}\left(d^{\prime}+d^{\prime \prime}-m-p\right)\right)} .
$$

The partial differential operators $\mathcal{D}_{d_{j}}^{(j)}$ (which depend also on $d_{1}, \ldots d_{n}$ ) are defined in terms of

$$
\mathcal{L}_{-p}^{(j)}:=-\sum_{i \neq j}\left(\left(x_{i}-x_{j}\right)^{1-p} \frac{\partial}{\partial x_{i}}+(1-p) h_{1, d_{i}}\left(x_{i}-x_{j}\right)^{-p}\right),
$$

by the Benoit \& Saint-Aubin formula

$$
\mathcal{D}_{d_{j}}^{(j)}:=\sum_{k=1}^{d_{j}} \sum_{\substack{p_{1}, \ldots, p_{k} \geq 1 \\ p_{1}+\cdots+p_{k}=d_{j}}} \frac{(-4 / \kappa)^{d_{j}-k}\left(d_{j}-1\right) !^{2}}{\prod_{u=1}^{k-1}\left(\sum_{i=1}^{u} p_{i}\right)\left(\sum_{i=u+1}^{k} p_{i}\right)} \times \mathcal{L}_{-p_{1}}^{(j)} \cdots \mathcal{L}_{-p_{k}}^{(j)} .
$$

The most important cases concern highest weight vectors, i.e., vectors $v$ satisfying $E . v=0$. We first state, however, the properties that do not depend on this assumption.

Theorem 4.16. Let $v \in \mathrm{M}_{d_{n}} \otimes \cdots \otimes \mathrm{M}_{d_{1}}$. The function $\left(x_{0} ; x_{1}, \ldots, x_{n}\right) \mapsto \mathcal{F}^{\left(x_{0}\right)}[v]\left(x_{1}, \ldots, x_{n}\right)$ satisfies the following properties:

(COV): For any $\xi \in \mathbb{R}$ we have the translation invariance

$$
\mathcal{F}^{\left(x_{0}+\xi\right)}[v]\left(x_{1}+\xi, \ldots, x_{n}+\xi\right)=\mathcal{F}^{\left(x_{0}\right)}[v]\left(x_{1}, \ldots, x_{n}\right) .
$$

If furthermore $K . v=q^{d-1} v$, then for any $\lambda>0$ we have the scaling covariance

$$
\mathcal{F}^{\left(\lambda x_{0}\right)}[v]\left(\lambda x_{1}, \ldots, \lambda x_{n}\right)=\lambda^{\Delta_{d}^{d_{1}, \ldots, d_{n}}} \times \mathcal{F}^{\left(x_{0}\right)}[v]\left(x_{1}, \ldots, x_{n}\right) .
$$

(ASY): If $v=\pi_{j, j+1}^{(d)}(v)$ and we denote

$$
\hat{v}=\hat{\pi}_{j, j+1}^{(d)}(v) \in\left(\bigotimes_{i=j+2}^{n} \mathrm{M}_{d_{i}}\right) \otimes \mathrm{M}_{d} \otimes\left(\bigotimes_{i=1}^{j-1} \mathrm{M}_{d_{i}}\right)
$$


then we have

$$
\begin{aligned}
\lim _{x_{j}, x_{j+1} \rightarrow \xi} & \left(\left(x_{j+1}-x_{j}\right)^{-\Delta_{d}^{d_{j}, d_{j+1}}} \times \mathcal{F}^{\left(x_{0}\right)}[v]\left(x_{1}, \ldots, x_{n}\right)\right) \\
= & B_{d}^{d_{j}, d_{j+1}} \times \mathcal{F}^{\left(x_{0}\right)}[\hat{v}]\left(x_{1}, \ldots, x_{j-1}, \xi, x_{j+2}, \ldots, x_{n}\right) .
\end{aligned}
$$

Proof. The statements (COV) about the translation invariance and homogeneity of $\mathcal{F}^{\left(x_{0}\right)}[v]\left(x_{1}, \ldots, x_{n}\right)$ are clear from the corresponding properties of the basis functions $\varphi_{l_{1}, \ldots, l_{n}}^{\left(x_{0}\right)}$ stated in Lemma 3.3 The statement (ASY) was proved in Proposition 4.4

In the case of highest weight vectors, the functions become independent of the anchor point $x_{0}$ and they solve Benoit \& Saint-Aubin partial differential equations. In the further special case of vectors in a trivial subrepresentation, one obtains full Möbius covariance. These properties are stated precisely in the following theorem.

Theorem 4.17. Assume that $v \in \mathrm{M}_{d_{n}} \otimes \cdots \otimes \mathrm{M}_{d_{1}}$ satisfies E. $v=0$. Then $\mathcal{F}^{\left(x_{0}\right)}[v]\left(x_{1}, \ldots, x_{n}\right)$ is independent of $x_{0}$, and thus defines a function $\mathcal{F}[v]$ on $\mathfrak{X}_{n}$. This function $\left(x_{1}, \ldots, x_{n}\right) \mapsto \mathcal{F}[v]\left(x_{1}, \ldots, x_{n}\right)$ satisfies the following properties:

(PDE): With $\mathcal{D}_{d_{j}}^{(j)}$ the differential operator 4.12, we have

$$
\mathcal{D}_{d_{j}}^{(j)} \mathcal{F}[v]=0 \quad \text { for } j=1, \ldots, n .
$$

(COV): For any $\xi \in \mathbb{R}$ we have the translation invariance

$$
\mathcal{F}[v]\left(x_{1}+\xi, \ldots, x_{n}+\xi\right)=\mathcal{F}[v]\left(x_{1}, \ldots, x_{n}\right) .
$$

If furthermore $K . v=q^{d-1} v$, then for any $\lambda>0$ we have the scaling covariance

$$
\mathcal{F}[v]\left(\lambda x_{1}, \ldots, \lambda x_{n}\right)=\lambda^{\Delta_{d}^{d_{1}, \ldots, d_{n}}} \times \mathcal{F}[v]\left(x_{1}, \ldots, x_{n}\right) .
$$

If furthermore $K . v=v$, then we have the full Möbius covariance

$$
\prod_{j=1}^{n} \mu^{\prime}\left(x_{j}\right)^{h_{1, d_{j}}} \times \mathcal{F}[v]\left(\mu\left(x_{1}\right), \ldots, \mu\left(x_{n}\right)\right)=\mathcal{F}[v]\left(x_{1}, \ldots, x_{n}\right)
$$

for any Möbius transformation $\mu: \mathbb{H} \rightarrow \mathbb{H}$ such that $\mu\left(x_{1}\right)<\mu\left(x_{2}\right)<\cdots<\mu\left(x_{n}\right)$.

(ASY): If $v=\pi_{j, j+1}^{(d)}(v)$ and we denote

$$
\hat{v}=\hat{\pi}_{j, j+1}^{(d)}(v) \in\left(\bigotimes_{i=j+2}^{n} \mathrm{M}_{d_{i}}\right) \otimes \mathrm{M}_{d} \otimes\left(\bigotimes_{i=1}^{j-1} \mathrm{M}_{d_{i}}\right),
$$

then also E. $\hat{v}=0$ and we have

$$
\begin{aligned}
\lim _{x_{j}, x_{j+1} \rightarrow \xi} & \left(\left(x_{j+1}-x_{j}\right)^{-\Delta_{d}^{d_{j}, d_{j+1}}} \times \mathcal{F}[v]\left(x_{1}, \ldots, x_{n}\right)\right) \\
= & B_{d}^{d_{j}, d_{j+1}} \times \mathcal{F}[\hat{v}]\left(x_{1}, \ldots, x_{j-1}, \xi, x_{j+2}, \ldots, x_{n}\right) .
\end{aligned}
$$

Proof. That $\mathcal{F}^{\left(x_{0}\right)}[v]$ does not depend on $x_{0}$ follows from Proposition 4.5 and Remark 4.6 after it — we may define

$$
\mathcal{F}[v]\left(x_{1}, \ldots, x_{n}\right)=\mathcal{F}^{\left(x_{0}\right)}[v]\left(x_{1}, \ldots, x_{n}\right) \quad \text { for any } x_{0}<x_{1} .
$$

The statement (PDE) was shown in Proposition 4.12 .

Of the statements (COV), the first two are direct consequences of the corresponding statements in Theorem 4.16 for $\mathcal{F}^{\left(x_{0}\right)}[v]$. The third statement was shown in Proposition 4.15

The statement (ASY) is a direct consequence of the fact that $\pi_{j, j+1}^{(d)}$ is a projection to a subrepresentation, and the corresponding statement in Theorem 4.16 for $\mathcal{F}^{\left(x_{0}\right)}[v]$. 
Remark 4.18. We have stated our main results assuming $q$ is not a root of unity. Let us, however, point out that certain root of unity cases work out completely parallel to the generic case.

For $q$ a root of unity, denote by $p$ the smallest positive integer such that $q^{p} \in\{ \pm 1\}$, or equivalently, the smallest positive integer such that $[p]=0$. Then the representations $\mathrm{M}_{d}$ are still irreducible if $d-1<p$, and moreover, the conclusion and formulas in the Clebsch-Gordan decomposition of Lemma 2.4 remain valid as long as $\left(d^{\prime}-1\right)+\left(d^{\prime \prime}-1\right)<p$. Using this repeatedly, one sees that the tensor product $\bigotimes_{j=1}^{n} \mathrm{M}_{d_{j}}$

used in the spin chain - Coulomb gas correspondence retains the generic direct sum decomposition as long as $\sum_{j=1}^{n}\left(d_{j}-1\right)<p$. It is also easy to check that, under the assumption $\sum_{j=1}^{n}\left(d_{j}-1\right)<p$, all formulas in Section 3 for the integral functions remain valid, and that they can be applied as we have done in this section to derive the above main theorems. In conclusion, while the general root of unity case may be very complicated (as discussed in Section 6), at least for $\sum_{j=1}^{n}\left(d_{j}-1\right)<p$ the statements need not be changed!

By this observation, for instance the pure partition functions $\mathcal{Z}_{\alpha}$ of multiple $\mathrm{SLE}_{\kappa}$ described in Section 1.4 .1 can be constructed for rational $\kappa \in(0,8) \cap \mathbb{Q}$ exactly like in the generic case, as long as the number $N$ of curves is sufficiently small, namely $N<\frac{p}{2}$ where $p=p(\kappa)$ is the smallest positive integer such that $\frac{4}{\kappa} p$ is an integer.

\section{FURTHER PROPERTIES}

In this section, we establish two more properties of the correspondence. First, in Section 5.1, we treat a generalization of the asymptotics property (ASY), to the case where more than two of the variables tend to a common limit. Then, in Section 5.2, we apply the general asymptotics statement to consider a limit where either the first or the last variable of a Möbius covariant function is taken to infinity. This property pertains to the manifestation of the periodicity of the boundary of a simply connected domain, discussed in Section 5.3 .

5.1. Asymptotics as several variables tend to a common limit. Suppose that $1 \leq j<k \leq n$. We first address what happens to the function

$$
\mathcal{F}^{\left(x_{0}\right)}[v]\left(x_{1}, \ldots, x_{j}, \ldots, x_{k}, \ldots, x_{n}\right)
$$

in the limit

$$
\begin{aligned}
x_{j}, x_{j+1}, \ldots, x_{k-1}, x_{k} & \rightarrow \xi & \text { taken in such a way that } \\
\frac{x_{i}-x_{j}}{x_{k}-x_{j}} & \rightarrow \eta_{i} & \text { for } i \in\{j, j+1, \ldots, k-1, k\} .
\end{aligned}
$$

We assume that $x_{j-1}<\xi<x_{k+1}$ and $0=\eta_{j}<\eta_{j+1}<\cdots<\eta_{k-1}<\eta_{k}=1$.

The case $k=j+1$, when only two points come together, was the content of part (ASY) of Theorems 4.17 and 4.16 . It was based on the decomposition

$$
\mathrm{M}_{d_{j+1}} \otimes \mathrm{M}_{d_{j}} \cong \bigoplus_{d} \mathrm{M}_{d}
$$

An important difference in the present case will be that in the decomposition

$$
\mathrm{M}_{d_{k}} \otimes \cdots \otimes \mathrm{M}_{d_{j}} \cong \bigoplus_{d} m_{d} \mathrm{M}_{d}
$$

some of the multiplicities $m_{d}$ may be greater than one. Note, however, that any vector in the space $m_{d} \mathrm{M}_{d} \subset \mathrm{M}_{d_{k}} \otimes \cdots \otimes \mathrm{M}_{d_{j}}$ may be written as a linear combination of vectors of the form $F^{l} . \tau_{0}$, where $0 \leq l<d$, and $\tau_{0}$ is some highest weight vector of an irreducible subrepresentation of dimension $d$ (there are $m_{d}$ linearly independent such highest weight vectors). It is therefore possible to deduce the behavior of the functions in general from the following result. 
Proposition 5.1. Suppose that $\tau_{0} \in \mathrm{M}_{d_{k}} \otimes \cdots \otimes \mathrm{M}_{d_{j}}$ satisfies E. $\tau_{0}=0$ and $K . \tau_{0}=q^{d-1} \tau_{0}$. Let

$$
\begin{array}{ll}
v=e_{l_{n}} \otimes \cdots \otimes e_{l_{k+1}} \otimes F^{l} \cdot \tau_{0} \otimes e_{l_{j-1}} \otimes \cdots \otimes e_{l_{1}} & \in \mathrm{M}_{d_{n}} \otimes \cdots \otimes \mathrm{M}_{d_{1}} \\
\hat{v}=e_{l_{n}} \otimes \cdots \otimes e_{l_{k+1}} \otimes e_{l} \otimes e_{l_{j-1}} \otimes \cdots \otimes e_{l_{1}} & \in \mathrm{M}_{d_{n}} \otimes \cdots \otimes \mathrm{M}_{d_{k+1}} \otimes \mathrm{M}_{d} \otimes \mathrm{M}_{d_{j-1}} \otimes \cdots \otimes \mathrm{M}_{d_{1}},
\end{array}
$$

and let $\Delta=\Delta_{d}^{d_{j}, \ldots, d_{k}}$ as in Section 4.8. Then in the limit (5.1) we have

$$
\frac{\mathcal{F}^{\left(x_{0}\right)}[v]\left(x_{1}, \ldots, x_{n}\right)}{\left|x_{k}-x_{j}\right|^{\Delta}} \longrightarrow \mathcal{F}\left[\tau_{0}\right]\left(\eta_{j}, \ldots \eta_{k}\right) \times \mathcal{F}^{\left(x_{0}\right)}[\hat{v}]\left(x_{1}, \ldots, x_{j-1}, \xi, x_{k+1}, \ldots, x_{n}\right) \text {. }
$$

We have preferred to formulate the above proposition for a vector $v$ of specific form. The reason is that the limit function's dependence on the ratios $\eta_{j}, \ldots, \eta_{k}$ depends on the $d$-dimensional irreducible subrepresentation whose highest weight vector is $\tau_{0}$. Note, however, that the exponent $\Delta=\Delta_{d}^{d_{j}, \ldots, d_{k}}$ in the asymptotics is the same for all $d$-dimensional subrepresentations of $\mathrm{M}_{d_{k}} \otimes \cdots \otimes \mathrm{M}_{d_{j}}$, and therefore a similar limit exists also more generally, as stated in the following.

Corollary 5.2. Suppose that $v \in \bigotimes_{i=1}^{n} \mathrm{M}_{d_{i}}$ belongs to the subrepresentation

$$
\left(\bigotimes_{i=k+1}^{n} \mathrm{M}_{d_{i}}\right) \otimes\left(m_{d} \mathrm{M}_{d}\right) \otimes\left(\bigotimes_{i=1}^{j-1} \mathrm{M}_{d_{i}}\right)
$$

Then the expression $\mathcal{F}^{\left(x_{0}\right)}[v]\left(x_{1}, \ldots, x_{n}\right) \times\left|x_{k}-x_{j}\right|^{-\Delta}$, with $\Delta=\Delta_{d}^{d_{j}, \ldots, d_{k}}$, has a limit 5.1).

Proof of Proposition 5.1. The proof follows a strategy parallel to the simpler asymptotics properties shown before. The crucial step is to rearrange the integrations to a form where there are deformed hypercube type contours between those variables which tend to a common limit, and all other contours are loops which either encircle one other variable, or encircle all the points with a common limit together. Once rearranged this way, dominated convergence theorem may be applied to complete the proof. The rearrangement itself is done in two steps, analogous to the two lemmas in Section 4.3. We only sketch the proofs, as the omitted details are reasonably straightforward modifications of the case already considered.

Analogously to Lemma 4.2 we first handle the case $l=0$ in which $\tau_{0}$ is a highest weight vector. We use the fact that the highest weight vector $\tau_{0}$ corresponds to a closed integration contour. This is expressed precisely in Proposition 4.5, which allows us to rearrange the integrals in $\mathcal{F}^{\left(x_{0}\right)}\left[\tau_{0}\right]\left(x_{j}, \ldots, x_{k}\right)$ so that no integration contour starts from $x_{0}$ :

$$
\left(\mathcal{F}^{\left(x_{0}\right)}\left[\tau_{0}\right]\right)\left(x_{j}, \ldots, x_{k}\right)=\sum c_{m_{j+1}, \ldots, m_{k}} \times \widetilde{\rho}_{0, m_{j+1}, \ldots, m_{k}}^{\left(x_{0}\right)}\left(x_{j}, \ldots, x_{k}\right) .
$$

The sum $m_{j+1}+\cdots+m_{k}$ of the indices is fixed, and it equals $\frac{1}{2}\left(\sum_{i=j}^{k} d_{i}-k+j-d\right)$. For the vector

$$
v=e_{l_{n}} \otimes \cdots \otimes e_{l_{k+1}} \otimes \tau_{0} \otimes e_{l_{j-1}} \otimes \cdots \otimes e_{l_{1}},
$$

by the same rearrangement of integrals, we obtain the expression

$$
\left(\mathcal{F}^{\left(x_{0}\right)}[v]\right)\left(x_{1}, \ldots, x_{n}\right)=\sum c_{m_{j+1}, \ldots, m_{k}} \times \alpha_{l_{1}, \ldots, l_{j-1} ; 0,\left\{m_{j+1}, \ldots, m_{k}\right\} ; l_{k+1}, \ldots, l_{n}}^{\left(x_{0}\right)}\left(x_{1}, \ldots, x_{n}\right),
$$

where $\alpha_{\ldots ; 0,\left\{m_{j+1}, \ldots, m_{k}\right\} ; \cdots}^{\left(x_{0}\right)}$ are generalizations of the mixed integrals $\alpha_{\ldots ; 0, m ; \cdots}^{\left(x_{0}\right)}$ defined in Section 3.4 the integration contours to variables $x_{j}, \ldots, x_{k}$ are as in $\widetilde{\rho}_{0, m_{j+1}, \ldots, m_{k}}^{\left(x_{0}\right)}$, and all other $x_{i}$ are encircled by $l_{i}$ non-intersecting nested loops based at the anchor point $x_{0}$.

The next step is to consider the case of general $l$, in a manner analogous to Lemma 4.3 . By comparison with the $(k-j)$-fold coproduct formula for the quantum group generator $F$ given in Lemma 2.2, one shows recursively in $l$ that for vectors

$$
v=e_{l_{n}} \otimes \cdots \otimes e_{l_{k+1}} \otimes F^{l} . \tau_{0} \otimes e_{l_{j-1}} \otimes \cdots \otimes e_{l_{1}},
$$

the integrals can be rearranged to

$$
\left(\mathcal{F}^{\left(x_{0}\right)}[v]\right)\left(x_{1}, \ldots, x_{n}\right)=\sum c_{m_{j+1}, \ldots, m_{k}} \times \alpha_{l_{1}, \ldots, l_{j-1} ; l,\left\{m_{j+1}, \ldots, m_{k}\right\} ; l_{k+1}, \ldots, l_{n}}^{\left(x_{0}\right)}\left(x_{1}, \ldots, x_{n}\right),
$$


where $\alpha_{\ldots ; l,\left\{m_{j+1}, \ldots, m_{k}\right\} ; \ldots}^{\left(x_{0}\right)}$ are generalizations of the mixed integrals $\alpha_{\ldots ; l, m ; \ldots}^{\left(x_{0}\right)}$. Compared to the case $l=0$, the new feature is that the variables $x_{j}, \ldots, x_{k}$ together with all integration contours connected to them are encircled by $l$ non-intersecting nested loops based at the anchor point $x_{0}$.

To reach the conclusion, we need to perform the limit 5.1] of $\alpha_{l_{1}, \ldots, l_{j-1} ; l,\left\{m_{j+1}, \ldots, m_{k}\right\} ; l_{k+1}, \ldots, l_{n}}^{\left(x_{n}\right)}$. Once we divide by $\left|x_{k}-x_{j}\right|^{\Delta}$, dominated convergence theorem can be applied to the integration over all variables whose contour is a loop, since these contours remain bounded away from the points $x_{j}, \ldots, x_{k}$ and any hypercube type integration contours between them. The loop type integration contours are the same as for $\mathcal{F}^{\left(x_{0}\right)}[\hat{v}]\left(x_{1}, \ldots, x_{j-1}, \xi, x_{k+1}, \ldots, x_{n}\right)$. The integral over the hypercube type contour divided by $\left|x_{k}-x_{j}\right|^{\Delta}$ tends to the integrand of $\mathcal{F}^{\left(x_{0}\right)}[\hat{v}]$ multiplied by $\mathcal{F}\left[\tau_{0}\right]\left(\eta_{j}, \ldots, \eta_{k}\right)$. The asserted result follows.

5.2. Moving one point to infinity. In the Möbius covariant case, we will now consider what happens to the function

$$
\mathcal{F}[v]\left(x_{1}, \ldots, x_{n}\right)
$$

as $x_{n} \rightarrow+\infty$. For this, we will need to be able to move from the trivial subrepresentation of $\bigotimes_{j=1}^{n} \mathrm{M}_{d_{j}}$ to the sum of copies of $d_{n}$-dimensional irreducible subrepresentations of $\bigotimes_{j=1}^{n-1} \mathrm{M}_{d_{j}}$.

Symmetrically, we consider what happens to the above function as $x_{1} \rightarrow-\infty$, in which case we will need to be able to move from the trivial subrepresentation of $\bigotimes_{j=1}^{n} \mathrm{M}_{d_{j}}$ to the sum of copies of $d_{1}$-dimensional irreducible subrepresentations of $\bigotimes_{j=2}^{n} \mathrm{M}_{d_{j}}$. The following lemma provides the needed mappings in the two cases.

Lemma 5.3. Let $\mathrm{H}_{1} \subset \bigotimes_{j=1}^{n} \mathrm{M}_{d_{j}}$ denote the trivial subrepresentation

$$
\mathrm{H}_{1}=\left\{v \in \bigotimes_{j=1}^{n} \mathrm{M}_{d_{j}} \mid E . v=0, K . v=v\right\} .
$$

(a) Any vector $v \in \mathrm{H}_{1}$ can be written uniquely in the form

$$
v=\sum_{l_{n}=0}^{d_{n}-1} A_{l_{n}}^{+} \times e_{l_{n}} \otimes\left(F^{d_{n}-1-l_{n}} \cdot \tau_{0}^{+}\right), \quad \text { with } A_{l_{n}}^{+}=(-1)^{d_{n}-1-l_{n}} q^{\left(l_{n}+1\right)\left(d_{n}-1-l_{n}\right)},
$$

where $\tau_{0}^{+} \in \bigotimes_{j=1}^{n-1} \mathrm{M}_{d_{j}}$ satisfies E. $\tau_{0}^{+}=0$ and $K . \tau_{0}^{+}=q^{d_{n}-1} \tau_{0}^{+}$. The mapping $v \mapsto \tau_{0}^{+}=R_{+}(v)$ defines a linear isomorphism $R_{+}: \mathrm{H}_{1} \rightarrow \mathrm{H}_{d_{n}}^{+}$to the space

$$
\mathrm{H}_{d_{n}}^{+}=\left\{\tilde{v} \in \bigotimes_{j=1}^{n-1} \mathrm{M}_{d_{j}} \mid E \cdot \tilde{v}=0, K . \tilde{v}=q^{d_{n}-1} \tilde{v}\right\}
$$

of highest weight vectors of irreducible subrepresentations of dimension $d_{n}$ in $\bigotimes_{j=1}^{n-1} \mathrm{M}_{d_{j}}$.

(b) Any vector $v \in \mathrm{H}_{1}$ can be written uniquely in the form

$$
v=\sum_{l_{1}=0}^{d_{1}-1} A_{l_{1}}^{-} \times\left(F^{d_{1}-1-l_{1}} \cdot \tau_{0}^{-}\right) \otimes e_{l_{1}}, \quad \text { with } A_{l_{1}}^{-}=(-1)^{d_{1}-1-l_{1}} q^{\left(l_{1}-1\right)\left(d_{1}-1-l_{1}\right)},
$$

where $\tau_{0}^{-} \in \bigotimes_{j=2}^{n} \mathrm{M}_{d_{j}}$ satisfies E. $\tau_{0}^{-}=0$ and $K . \tau_{0}^{-}=q^{d_{1}-1} \tau_{0}^{-}$. The mapping $v \mapsto \tau_{0}^{-}=R_{-}(v)$ defines a linear isomorphism $R_{-}: \mathrm{H}_{1} \rightarrow \mathrm{H}_{d_{1}}^{-}$to the space

$$
\mathrm{H}_{d_{1}}^{-}=\left\{\tilde{v} \in \bigotimes_{j=2}^{n} \mathrm{M}_{d_{j}} \mid E \cdot \tilde{v}=0, K . \tilde{v}=q^{d_{1}-1} \tilde{v}\right\}
$$

of highest weight vectors of irreducible subrepresentations of dimension $d_{1}$ in $\bigotimes_{j=2}^{n} \mathrm{M}_{d_{j}}$. 
Proof. The two parts are similar, so we only give the details for part (a). Any vector $v$ in the tensor product $\bigotimes_{j=1}^{n} \mathrm{M}_{d_{j}}$ can be written in the form $v=\sum_{l_{n}} e_{l_{n}} \otimes u_{l_{n}}$ with unique vectors $u_{l_{n}} \in \bigotimes_{j=1}^{n-1} \mathrm{M}_{d_{j}}$. From the eigenvalue property $K \cdot e_{l_{n}}=q^{d_{n}-1-2 l_{n}} e_{l_{n}}$ and the coproduct formula $\Delta(K)=K \otimes K$, it follows that we have

$$
K . v=\sum_{l_{n}} q^{d_{n}-1-2 l_{n}} \times e_{l_{n}} \otimes\left(K . u_{l_{n}}\right) .
$$

The assumption $K . v=v$ thus implies that we have $K \cdot u_{l_{n}}=q^{1-d_{n}+2 l_{n}} u_{l_{n}}$. Similarly, from the property $E . e_{l_{n}}=\left[l_{n}\right]\left[d_{n}-l_{n}\right] e_{l_{n}-1}$, and the coproduct formula $\Delta(E)=E \otimes K+1 \otimes E$, and the already established $K$-eigenvalue of $u_{l_{n}}$, it follows that we have

$$
E . v=\sum_{l_{n}} e_{l_{n}} \otimes\left(E . u_{l_{n}}+\left[l_{n}+1\right]\left[d_{n}-l_{n}-1\right] q^{3-d_{n}+2 l_{n}} u_{l_{n}+1}\right) .
$$

The assumption E.v $=0$ thus implies E. $u_{l_{n}}=-\left[l_{n}+1\right]\left[d_{n}-l_{n}-1\right] q^{3-d_{n}+2 l_{n}} u_{l_{n}+1}$. In particular, $u_{d_{n}-1}$ is a highest weight vector of an irreducible subrepresentation of dimension $d_{n}$. We denote this vector by $u_{d_{n}-1}=\tau_{0}^{+}$. We furthermore claim that this vector determines the other $u_{l_{n}}$ uniquely as $u_{l_{n}}=A_{l_{n}}^{+} F^{d_{n}-1-l_{n}} \cdot \tau_{0}^{+}$. To see this, use the coproduct formula $\Delta(F)=F \otimes 1+K^{-1} \otimes F$, to get

$$
F . v=\sum_{l_{n}} e_{l_{n}} \otimes\left(u_{l_{n}-1}+q^{1-d_{n}+2 l_{n}} F \cdot u_{l_{n}}\right) .
$$

Note that this expression for F.v must vanish, since $v$ is in the trivial subrepresentation. Therefore the other $u_{l_{n}}$ are obtained recursively from $u_{d_{n}-1}=\tau_{0}^{+}$, by $u_{l_{n}-1}=-q^{1-d_{n}+2 l_{n}} F \cdot u_{l_{n}}$. The solution of this recursion is the asserted formula $u_{l_{n}}=A_{l_{n}}^{+} F^{d_{n}-1-l_{n}} \cdot \tau_{0}^{+}$, and since this formula indeed satisfies E.v $=0$ and $K \cdot v=v$, we get that the linear mapping $R_{+}$defined by $R_{+}(v)=u_{d_{n}-1}$ is bijective.

We now show that the behavior of Möbius covariant functions as $x_{n} \rightarrow+\infty$ (resp. $x_{1} \rightarrow-\infty$ ) can be expressed in terms of the identification $R_{+}$(resp. $R_{-}$) defined in Lemma 5.3.

Proposition 5.4. Let $v \in \mathrm{H}_{1} \subset \bigotimes_{j=1}^{n} \mathrm{M}_{d_{j}}$, and use the mappings defined in Lemma 5.3 to construct $R_{+}(v) \in \mathrm{H}_{d_{n}}^{+} \subset \bigotimes_{j=1}^{n-1} \mathrm{M}_{d_{j}}$ and $R_{-}(v) \in \mathrm{H}_{d_{1}}^{-} \subset \bigotimes_{j=2}^{n} \mathrm{M}_{d_{j}}$. Then we have

$$
\lim _{y \rightarrow+\infty}\left(y^{2 h_{1, d_{n}}} \times \mathcal{F}[v]\left(x_{1}, \ldots, x_{n-1}, y\right)\right)=C_{+} \times \mathcal{F}\left[R_{+}(v)\right]\left(x_{1}, \ldots, x_{n-1}\right),
$$

with $C_{+}=\left(q-q^{-1}\right)^{d_{n}-1}\left[d_{n}-1\right] !^{2} \times B_{1}^{d_{n}, d_{n}}$ and

$$
\lim _{y \rightarrow-\infty}\left(|y|^{2 h_{1, d_{1}}} \times \mathcal{F}[v]\left(y, x_{2}, \ldots, x_{n}\right)\right)=C_{-} \times \mathcal{F}\left[R_{-}(v)\right]\left(x_{2}, \ldots, x_{n}\right) .
$$

with $C_{-}=\left(q^{-2}-1\right)^{d_{1}-1}\left[d_{1}-1\right] !^{2} \times B_{1}^{d_{1}, d_{1}}$.

Proof. Again, the two cases are similar, so we only give the details about the first. We write the vector $v$ in the form given by Lemma 5.3(a), as $v=\sum_{l_{n}} A_{l_{n}}^{+} \times e_{l_{n}} \otimes F^{d_{n}-1-l_{n}} \cdot \tau_{0}^{+}$, where $\tau_{0}^{+}=R_{+}(v)$ is a highest weight vector of a $d_{n}$-dimensional subrepresentation of $\bigotimes_{j=1}^{n-1} \mathrm{M}_{d_{j}}$. We then write, using homogeneity,

$$
y^{2 h_{1, d_{n}}} \times \mathcal{F}[v]\left(x_{1}, \ldots, x_{n-1}, y\right)=y^{h_{1, d_{n}}-\sum_{j=1}^{n-1} h_{1, d_{j}}} \times \mathcal{F}[v]\left(\frac{x_{1}}{y}, \ldots, \frac{x_{n-1}}{y}, 1\right) .
$$

We apply Proposition 5.1 to this. More precisely, for all terms $e_{l_{n}} \otimes F^{d_{n}-1-l_{n}} \cdot \tau_{0}^{+}$we have

$$
\begin{aligned}
& \lim _{y \rightarrow+\infty}\left(y^{2 h_{1, d_{n}}} \times \mathcal{F}^{\left(x_{0}\right)}\left[e_{l_{n}} \otimes F^{d_{n}-1-l_{n}} \cdot \tau_{0}^{+}\right]\left(x_{1}, \ldots, x_{n-1}, y\right)\right) \\
= & \lim _{y \rightarrow+\infty}\left(y^{h_{1, d_{n}}-\sum_{j=1}^{n-1} h_{1, d_{j}}} \times \mathcal{F}^{\left(x_{0}\right)}\left[e_{l_{n}} \otimes F^{d_{n}-1-l_{n}} \cdot \tau_{0}^{+}\right]\left(\frac{x_{1}}{y}, \ldots, \frac{x_{n-1}}{y}, 1\right)\right) \\
= & \mathcal{F}\left[\tau_{0}^{+}\right]\left(x_{1}, \ldots, x_{n-1}\right) \times \mathcal{F}^{\left(x_{0}\right)}\left[e_{l_{n}} \otimes e_{d_{n}-1-l_{n}}\right](0,1) .
\end{aligned}
$$


Now the vector $v$ reads $v=\sum_{l_{n}} A_{l_{n}}^{+} \times e_{l_{n}} \otimes F^{d_{n}-1-l_{n}} \cdot \tau_{0}^{+}$, so by linearity we have

$$
\begin{aligned}
\lim _{y \rightarrow+\infty}\left(y^{2 h_{1, d_{n}}} \times \mathcal{F}[v]\left(x_{1}, \ldots, x_{n-1}, y\right)\right) & =\mathcal{F}\left[\tau_{0}^{+}\right]\left(x_{1}, \ldots, x_{n-1}\right) \times \sum_{l_{n}} A_{l_{n}}^{+} \times \mathcal{F}^{\left(x_{0}\right)}\left[e_{l_{n}} \otimes e_{d_{n}-1-l_{n}}\right](0,1) \\
& =\left(q-q^{-1}\right)^{d_{n}-1}\left[d_{n}-1\right] !^{2} \times B_{1}^{d_{n}, d_{n}} \times \mathcal{F}\left[\tau_{0}^{+}\right]\left(x_{1}, \ldots, x_{n-1}\right),
\end{aligned}
$$

where in the last step we used the facts that $\sum_{l_{n}} A_{l_{n}}^{+} \times e_{l_{n}} \otimes e_{d_{n}-1-l_{n}}=\left(q-q^{-1}\right)^{d_{n}-1}\left[d_{n}-1\right] !^{2} \times \tau_{0}^{\left(1 ; d_{n}, d_{n}\right)}$ in the notation of Equation 2.8, and $\mathcal{F}^{\left(x_{0}\right)}\left[\tau_{0}^{\left(1 ; d_{n}, d_{n}\right)}\right](0,1)=B_{1}^{d_{n}, d_{n}}$, by Proposition 4.4

Remark 5.5. Proposition 5.4 can be seen in two ways: it allows us to trade Möbius covariance to dependence on one variable less. Directly by the statement, any Möbius covariant function of n variables may be viewed as a function of $n-1$ variables - we can either get rid of the variable $x_{1}$ or $x_{n}$. Conversely, if we assume that $v \in \bigotimes_{j=1}^{n} \mathrm{M}_{d_{j}}$ lies in a d-dimensional irreducible subrepresentation, then we can view it as a Möbius covariant function of $n+1$ variables - either associated to the vector $R_{+}^{-1}(v) \in \mathrm{M}_{d} \otimes\left(\bigotimes_{j=1}^{n} \mathrm{M}_{d_{j}}\right)$ or to the vector $R_{-}^{-1}(v) \in\left(\bigotimes_{j=1}^{n} \mathrm{M}_{d_{j}}\right) \otimes \mathrm{M}_{d}$, and with the additional variable respectively to the right or to the left of all other variables.

5.3. Cyclic permutations of variables. Remark 5.5 suggests yet another interesting way to interpret the operations $R_{+}$and $R_{-}$in Lemma 5.3. Namely, in view of Proposition 5.4, the composed map $S=R_{-}^{-1} \circ R_{+}$gives rise to cyclic permutations of variables in our functions.

Consider the Möbius covariant case, and denote by $H_{1}(V)$ the maximal trivial subrepresentation of a representation $V$. Let $v$ be in the trivial subrepresentation $H_{1}\left(\mathrm{M}_{d_{n}} \otimes \mathrm{M}_{d_{n-1}} \otimes \cdots \otimes \mathrm{M}_{d_{1}}\right)$. Then the vector $S(v)$ is in the trivial subrepresentation $H_{1}\left(\mathrm{M}_{d_{n-1}} \otimes \cdots \otimes \mathrm{M}_{d_{1}} \otimes \mathrm{M}_{d_{n}}\right)$, and the function $\mathcal{F}[S(v)]$ could be thought of as corresponding to the original function $\mathcal{F}[v]$ when the variables are ordered as $x_{n}<x_{1}<x_{2}<\cdots<x_{n-1}$.

When the number of variables is $n$, and the operation of moving the rightmost variable to the left of all others is repeated $n$ times, one expects to recover the original function. Above we have defined this operation by its action on vectors in the trivial subrepresentation of the $n$-fold tensor product as

$$
S^{\left(d_{n}\right)}: H_{1}\left(\mathrm{M}_{d_{n}} \otimes \mathrm{M}_{d_{n-1}} \otimes \cdots \otimes \mathrm{M}_{d_{1}}\right) \rightarrow H_{1}\left(\mathrm{M}_{d_{n-1}} \otimes \cdots \otimes \mathrm{M}_{d_{1}} \otimes \mathrm{M}_{d_{n}}\right),
$$

where we now emphasize that the definition of the operation $S^{\left(d_{n}\right)}=R_{-}^{-1} \circ R_{+}$depends on the dimension $d_{n}$. The correct $n$ :th iterate is thus

$$
S^{\left(d_{1}\right)} \circ S^{\left(d_{2}\right)} \circ \cdots \circ S^{\left(d_{n-1}\right)} \circ S^{\left(d_{n}\right)}: H_{1}\left(\bigotimes_{j=1}^{n} \mathrm{M}_{d_{j}}\right) \rightarrow H_{1}\left(\bigotimes_{j=1}^{n} \mathrm{M}_{d_{j}}\right) .
$$

This mapping turns out to be not exactly the identity, but rather a constant multiple of the identity.

To see that $S^{\left(d_{n}\right)} \circ \cdots \circ S^{\left(d_{1}\right)}$ is a constant multiple of the identity on $H_{1}\left(\bigotimes_{j=1}^{n} \mathrm{M}_{d_{j}}\right)$, it is convenient to characterize the vectors in $H_{1}\left(\bigotimes_{j=1}^{n} \mathrm{M}_{d_{j}}\right)$ by their projections to different subrepresentations. One then needs two basic commutative diagrams, which express how to exchange the order of the projections and the operations $S^{\left(d_{j}\right)}$. Let

$$
\hat{\pi}_{j, j+1}^{(\delta)}: \bigotimes_{i=1}^{n} \mathrm{M}_{d_{j}} \rightarrow\left(\bigotimes_{i=j+2}^{n} \mathrm{M}_{d_{j}}\right) \otimes \mathrm{M}_{\delta} \otimes\left(\bigotimes_{i=1}^{j-1} \mathrm{M}_{d_{j}}\right)
$$

denote the projection $\mathrm{M}_{d_{j+1}} \otimes \mathrm{M}_{d_{j}} \rightarrow \mathrm{M}_{\delta}$ acting in the $j$ :th and $j+1$ :st tensorands, as in Section 4.3 If $j<n-1$, then the projection obviously commutes with the operation $S^{\left(d_{n}\right)}$, according to the following 
square diagram

$$
\begin{gathered}
H_{1}\left(\mathrm{M}_{d_{n}} \otimes \mathrm{M}_{d_{n-1}} \otimes \cdots \otimes \mathrm{M}_{d_{1}}\right) \\
\left.S^{\left(d_{n}\right)}\right|_{\downarrow} \stackrel{\hat{\pi}_{j, j+1}^{(\delta)}}{\longrightarrow} H_{1}\left(\mathrm{M}_{d_{n}} \otimes \mathrm{M}_{d_{n-1}} \otimes \cdots \otimes \mathrm{M}_{\delta} \otimes \cdots \otimes \mathrm{M}_{d_{1}}\right) \\
H_{1}\left(\mathrm{M}_{d_{n-1}} \otimes \cdots \otimes \mathrm{M}_{d_{1}} \otimes \mathrm{M}_{d_{n}}\right) \underset{S^{\left(d_{n}\right)}}{\underset{\hat{\pi}_{j+1, j+2}^{(\delta)}}{\longrightarrow}} H_{1}\left(\mathrm{M}_{d_{n-1}} \otimes \cdots \otimes \mathrm{M}_{\delta} \otimes \cdots \otimes \mathrm{M}_{d_{1}} \otimes \mathrm{M}_{d_{n}}\right) .
\end{gathered}
$$

If $j=n-1$, then a diagram of the above type does not make sense — instead, we apply two $S$-operations before the projection. The following pentagon diagram commutes up to a multiplicative constant

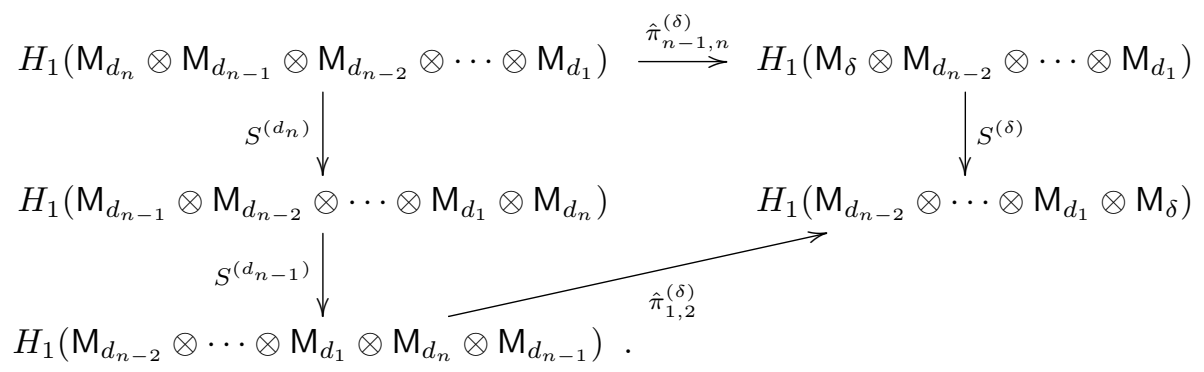

Using the above square and pentagon diagrams $\sqrt{5.2}$ and $\sqrt{5.3}$, one can finally show that also the larger diagram (5.4) below commutes up to constants. We choose a sequence of intermediate dimensions $\delta_{2}, \delta_{3}, \ldots, \delta_{n-2}, \delta_{n-1}=d_{n}$, to specify an element of the dual of $H_{1}\left(\bigotimes_{i=1}^{n} \mathrm{M}_{d_{i}}\right)$ through a sequence of projections. In this diagram, the top left $H_{1}$ stands for $H_{1}\left(\bigotimes_{i=1}^{n} \mathrm{M}_{d_{i}}\right)$, and moving downwards amounts to a cyclic permutation of the tensorands by an $S$-operation, and moving to the right reduces the number of tensorands by a projection. In the rightmost column, only two tensorands remain and the spaces $H_{1}$ stand for $H_{1}\left(\mathrm{M}_{d_{n}} \otimes \mathrm{M}_{d_{n}}\right)$ - note that $\delta_{n-1}=d_{n}$, as is needed for $\mathrm{M}_{d_{n}} \otimes \mathrm{M}_{\delta_{n-1}}$ to contain a trivial 
subrepresentation (see Lemma 2.4). The diagram

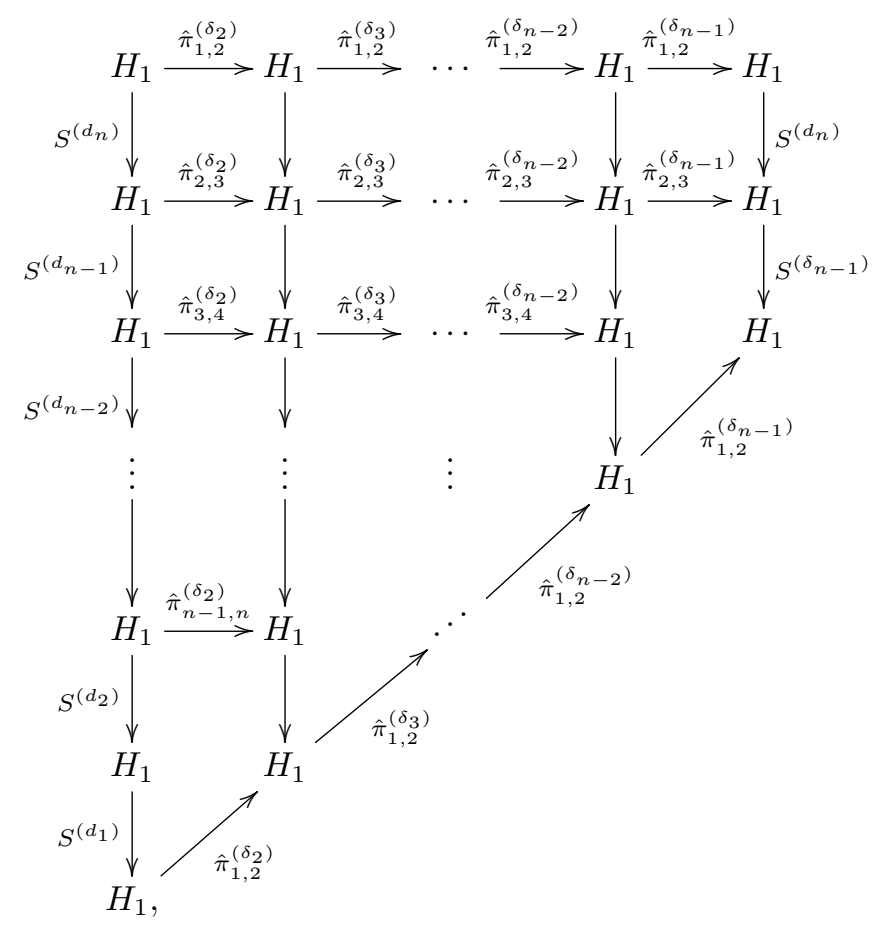

commutes up to constants, and we then deduce that

$$
\hat{\pi}_{1,2}^{\left(\delta_{n-1}\right)} \circ \cdots \circ \hat{\pi}_{1,2}^{\left(\delta_{2}\right)}=C \times \hat{\pi}_{1,2}^{\left(\delta_{n-1}\right)} \circ \cdots \circ \hat{\pi}_{1,2}^{\left(\delta_{2}\right)} \circ S^{\left(d_{1}\right)} \circ \cdots \circ S^{\left(d_{n}\right)} .
$$

The constant $C$ above is independent of the sequence of dimensions $\delta_{2}, \ldots, \delta_{n-1}$. Such projections span the dual of $H_{1}\left(\bigotimes_{i=1}^{n} \mathrm{M}_{d_{i}}\right)$, which allows us to conclude that

$$
S^{\left(d_{1}\right)} \circ \cdots \circ S^{\left(d_{n}\right)}=C \times \operatorname{id}_{H_{1}\left(\bigotimes_{i=1}^{n} \mathrm{M}_{d_{i}}\right)} .
$$

Thus, the $S$-operations give rise to a projective action of cyclic permutations on our Möbius covariant functions $\mathcal{F}[v]: \mathfrak{X}_{n} \rightarrow \mathbb{C}$. The constant is explicitly calculated in [Pel18, Corollary 4.3]: $C=\prod_{i=1}^{n}(-q)^{1-d_{i}}$.

\section{Conclusions And outlook}

We have defined the spin chain - Coulomb gas correspondence, which associates screened Coulomb gas correlation functions to vectors in a tensor product of representations of the quantum group $\mathcal{U}_{q}\left(\mathfrak{s l}_{2}\right)$. Natural representation theoretical properties of the vectors have been shown to imply properties of the corresponding functions.

The results presented here are used in [JJK16, KP16] to explicitly solve two interesting problems about SLEs. Other conformally covariant boundary correlation functions could be treated similarly with the techniques of the present article. Also, in [FP18b], the spin chain - Coulomb gas correspondence is applied to the construction of monodromy invariant bulk correlation functions of conformal field theory.

The results of the present article apply in the generic, semisimple case, in which the deformation parameter $q$ of the quantum group is not a root of unity. If $q$ were a root of unity - that is, $\kappa \in \mathbb{Q}-$ the representation theory of the quantum group would become non-semisimple and the corresponding functions would have degeneracies (exceptional linear dependencies or poles as a function of $\kappa$ ). Extending a version of the spin chain - Coulomb gas correspondence to these degenerate cases is a natural 
topic of future research - some of the non-semisimple representation theory has been analyzed in, e.g., [BFGT09], and examples of degeneracies of the functions have been resolved in, e.g., [FK15c, FSK15.

The boundary correlation functions obtained in this correspondence have conformal weights labeled by the first row of the Kac table. This is sufficient for those applications that served as our primary motivation, but the correspondence could possibly be generalized by considering another type of screening charges, and an appropriate "two-screening quantum group" [DF84, Fuc92].

\section{Appendix A. Contour manipulations}

In this appendix, we collect the proofs of intermediate results that involve in principle straightforward but occasionally lengthy contour deformation and branch choice calculations.

Lemma (Lemma 3.2. The deformed hypercube integral function $\widetilde{\rho}_{m_{1}, \ldots, m_{n}}^{\left(x_{0}\right)}$ defined in 3.5 is related to the real integral function $\rho_{m_{1}, \ldots, m_{n}}^{\left(x_{0}\right)}$ defined in 3.3 by

$$
\widetilde{\rho}_{m_{1}, \ldots, m_{n}}^{\left(x_{0}\right)}(\boldsymbol{x})=\left(\prod_{i=1}^{n} q^{-\left(\begin{array}{c}
m_{i} \\
2
\end{array}\right)}\left[m_{i}\right] !\right) \times \rho_{m_{1}, \ldots, m_{n}}^{\left(x_{0}\right)}(\boldsymbol{x}), \quad \text { for } \boldsymbol{x} \in \mathfrak{X}_{n}^{\left(x_{0}\right)}
$$

Proof. Note that $\rho$ is obtained by integration over the set $\mathcal{R}_{m_{1}, \ldots, m_{n}}$ which is a product of simplices, whereas $\widetilde{\rho}$ is obtained by integration over the set $\widetilde{\mathcal{R}}_{m_{1}, \ldots, m_{n}}$ which is a product of slightly deformed hypercubes. We split each of the $m_{i}$-dimensional hypercubes to $m_{i} !$ simplices, and thus express $\widetilde{\rho}_{m_{1}, \ldots, m_{n}}^{\left(x_{0}\right)}$ as a sum of $\prod_{i=1}^{n}\left(m_{i} !\right)$ terms, each of which is a phase factor times $\rho_{m_{1}, \ldots, m_{n}}^{\left(x_{0}\right)}$.

The variables $w_{r}, r \in I^{(i)}$, are integrated over one of the slightly deformed hypercubes. We select the deformed hypercube integration contour so that the variables are on the real axis, except when the distance between some of the variables becomes smaller than a chosen $\varepsilon>0$. In view of the integrand, proportional to (3.1), the contribution from cases with some $\left|w_{r}-x_{i}\right|<\varepsilon$ is $\mathcal{O}\left(\varepsilon^{1-\frac{4}{\kappa} \max \left(d_{i}-1\right)}\right)$, and the further contribution from cases with some $\left|w_{s}-w_{r}\right|<\varepsilon$ is $\mathcal{O}\left(\varepsilon^{1+\frac{8}{\kappa}}\right)$. We may thus neglect these contributions, which tend to zero as $\varepsilon \searrow 0$, and only consider cases with variables $w_{r}$ on the real line in some definite order.

We encode the possible orderings of the variables $w_{r}$ by $n$-tuples $\left(\sigma^{(1)}, \ldots, \sigma^{(n)}\right)$, where $\sigma^{(i)}$ is a permutation of $I^{(i)}$ : the associated order of the variables is

$$
\begin{array}{ll}
w_{\sigma^{(i)}(r)}<w_{\sigma^{(i)}\left(r^{\prime}\right)} & \text { for all } r, r^{\prime} \in I^{(i)} \text { such that } r<r^{\prime}, \\
w_{\sigma^{(i)}(r)}<w_{\sigma^{(j)}\left(r^{\prime}\right)} & \text { for all } r \in I^{(i)}, r^{\prime} \in I^{(j)} \text { such that } i<j .
\end{array}
$$

By our definition, the phase of $f_{m_{1}, \ldots, m_{n}}$ is positive when the ordering of the variables is the one corresponding to all identity permutations, $\sigma^{(i)}=\operatorname{id}_{I^{(i)}}$ for $i=1, \ldots, n$. In the limit $\varepsilon \searrow 0$, the integration over the set where the variables respect this standard ordering thus simply reproduces $\rho_{m_{1}, \ldots, m_{n}}^{\left(x_{0}\right)}$. If the ordering among the variables $w_{r}, r \in I^{(i)}$, is given by some other permutation $\sigma^{(i)}$, then the phase factors accumulated from exchanging the orders of these variables is $q^{-2 \times \# \operatorname{inv}\left(\sigma^{(i)}\right)}$, where

$$
\operatorname{inv}\left(\sigma^{(i)}\right)=\left\{r, s \in I^{(i)} \mid r<s \text { and } \sigma^{(i)}(r)>\sigma^{(i)}(s)\right\}
$$

denotes the set of inversions of $\sigma^{(i)}$. Apart from these phase factors, the contribution of the integral from the set corresponding to the ordering $\left(\sigma^{(1)}, \ldots, \sigma^{(n)}\right)$ coincides with the integral over the standard ordered part. In conclusion, we have

$$
\widetilde{\rho}_{m_{1}, \ldots, m_{n}}^{\left(x_{0}\right)}=\sum_{\sigma^{(1)}, \ldots, \sigma^{(n)}}\left(\prod_{i=1}^{n} q^{-2 \times \# \operatorname{inv}\left(\sigma^{(i)}\right)}\right) \times \rho_{m_{1}, \ldots, m_{n}}^{\left(x_{0}\right)} .
$$

By Lemma 2.1 (b) we simplify the prefactor to the asserted form. 
Lemma (Lemma 3.5). In the case $n=1$, the basis function (3.6) is related to the real integral by

$$
\varphi_{\ell}^{\left(x_{0}\right)}(x)=\left([\ell] ! \prod_{m=1}^{\ell}\left(q^{d-m}-q^{m-d}\right)\right) \times \rho_{\ell}^{\left(x_{0}\right)}(x) .
$$

In particular, $\varphi_{\ell}^{\left(x_{0}\right)}$ is identically zero if $\ell \geq d$.

Proof. We will prove that $\varphi_{\ell}^{\left(x_{0}\right)}(x)=q^{\left(\begin{array}{l}\ell \\ 2\end{array}\right)}\left(\prod_{m=1}^{\ell}\left(q^{d-m}-q^{m-d}\right)\right) \times \widetilde{\rho}_{\ell}^{\left(x_{0}\right)}(x)$ and the asserted equality will then follow from Lemma 3.2 . We achieve this by decomposing each of the loops based at $x_{0}$ encircling $x$ to two pieces: one from $x_{0}$ to $x$ and the other from $x$ back to $x_{0}$. We begin from the innermost loop and inductively proceed outwards. The procedure is illustrated in Figure A.1.

Let $\mathcal{C}_{1}, \ldots, \mathcal{C}_{\ell}$ be the contours of integration of the variables $w_{1}, \ldots, w_{\ell}$, so that $\mathcal{C}_{1}$ is a loop encircling $x$, and for $r>1$ the loop $\mathcal{C}_{r}$ encircles the entire loop $\mathcal{C}_{r-1}$. The basis function then reads

$$
\varphi_{\ell}^{\left(x_{0}\right)}(x)=\int_{\mathcal{C}_{\ell}} \mathrm{d} w_{\ell} \cdots \int_{\mathcal{C}_{2}} \mathrm{~d} w_{2} \int_{\mathcal{C}_{1}} \mathrm{~d} w_{1} f_{\ell}^{\ni}\left(x ; w_{1}, \ldots, w_{\ell}\right),
$$

see also Figure A.1(a), Recall that the branch and phase of the integrand are chosen so that

$$
f_{\ell}^{\ni}\left(x ; w_{1}, \ldots, w_{\ell}\right)=\prod_{r=1}^{\ell}\left(w_{r}-x\right)^{\frac{4}{\kappa}(1-d)} \prod_{1 \leq r<s \leq \ell}\left(w_{s}-w_{r}\right)^{\frac{8}{\kappa}}
$$

is positive at the "midpoint" $\boldsymbol{w}^{\prime}$ of the loops (illustrated by the red dots in the figure). We may thus fix the branches of each of the factors above so that they are positive when $x<w_{1}<w_{2}<\cdots<w_{\ell}$.

Choose a small $\varepsilon>0$. To decompose the first loop, that is, the innermost integral

$$
\int_{\mathcal{C}_{1}} f_{\ell}^{\ni} \mathrm{d} w_{1}
$$

deform it to a line segment along the real axis from $x_{0}$ to $x-\varepsilon$, a circle of radius $\varepsilon$ around $x$, and a line segment along the real axis from $x-\varepsilon$ to $x_{0}$. The innermost integral then becomes essentially

$$
\int_{x_{0}}^{x-\varepsilon} f_{\bar{\ell}}^{\ni} \mathrm{d} w_{1}+\oint_{\partial B_{\varepsilon}(x)} f_{\bar{\ell}}^{\ni} \mathrm{d} w_{1}+\int_{x-\varepsilon}^{x_{0}} f_{\ell}^{\ni} \mathrm{d} w_{1},
$$

but in this expression we have abused notation and hidden the important phase factors. In particular, the first and last terms do not cancel - the factor $\left(x-w_{1}\right)^{\frac{4}{\kappa}(1-d)}$ in the integrand $f_{\ell}^{\ni}$ has a constant but different phase on these two line segments. Indeed, on the first line segment $\left(x-w_{1}\right)^{\frac{4}{\kappa}(1-d)}=$ $q^{d-1}\left|x-w_{1}\right|^{\frac{4}{\kappa}(1-d)}$ and on the second $\left(x-w_{1}\right)^{\frac{4}{\kappa}(1-d)}=q^{1-d}\left|x-w_{1}\right|^{\frac{4}{\kappa}(1-d)}$, because from the reference point $\boldsymbol{w}^{\prime}$ we must take $w_{1}$ half a turn around $x$ in the negative or positive direction, respectively. The contribution of the integral around the circle is proportional to $\varepsilon^{1-\frac{4}{\kappa}(d-1)}$ and it thus vanishes in the limit $\varepsilon \searrow 0$ when $\kappa>4(d-1)$. Hence, we can write

$$
\int_{\mathcal{C}_{1}} \mathrm{~d} w_{1} f_{\ell}^{\ni}\left(x ; w_{1}, \ldots, w_{\ell}\right)=\left(q^{d-1}-q^{1-d}\right) \int_{x_{0}}^{x} \mathrm{~d} w_{1}\left|x-w_{1}\right|^{\frac{4}{\kappa}(1-d)} \prod_{r=2}^{\ell}\left(w_{r}-x\right)^{\frac{4}{\kappa}(1-d)} \prod_{1 \leq r<s \leq \ell}\left(w_{s}-w_{r}\right)^{\frac{8}{\kappa}} .
$$

These steps are illustrated in Figure A.1(b).

We proceed similarly with the integration contours $\mathcal{C}_{2}, \ldots, \mathcal{C}_{\ell}$, cutting each of them into two pieces between $x_{0}$ and $x$, and a negligible loop around $x$. The example of the second loop $\mathcal{C}_{2}$ is illustrated in Figure A.1(c), On each piece of the $r$ :th loop $\mathcal{C}_{r}$, we rephase the factors of the integrand that contain $w_{r}$, in order to finally compare with the integral $\widetilde{\rho}$. The first piece of the integration contour $\mathcal{C}_{r}$ is a path from $x_{0}$ to $x$ below the variables $w_{s}$, for $s<r$, and we extract a phase factor $q^{d-1}$ resulting from taking $w_{r}$ half a turn around $x$ in the negative direction. The second piece of the integration contour $\mathcal{C}_{r}$ is a path from $x$ to $x_{0}$ above the variables $w_{s}$, for $s<r$, and we not only take $w_{r}$ in the positive direction around $x$, but we also take it positively around all $w_{s}, s<r$, in order to reach a position where the 


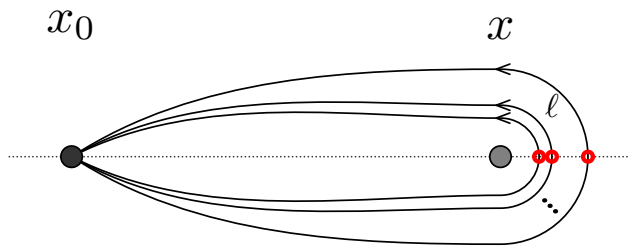

(a) The proof of Lemma 3.5 consists of manipulating the integral of $\varphi_{\ell}^{\left(x_{0}\right)}(x)$, which has $\ell$ nonintersecting positively oriented loops around $x$, anchored at $x_{0}$, as illustrated in this figure.

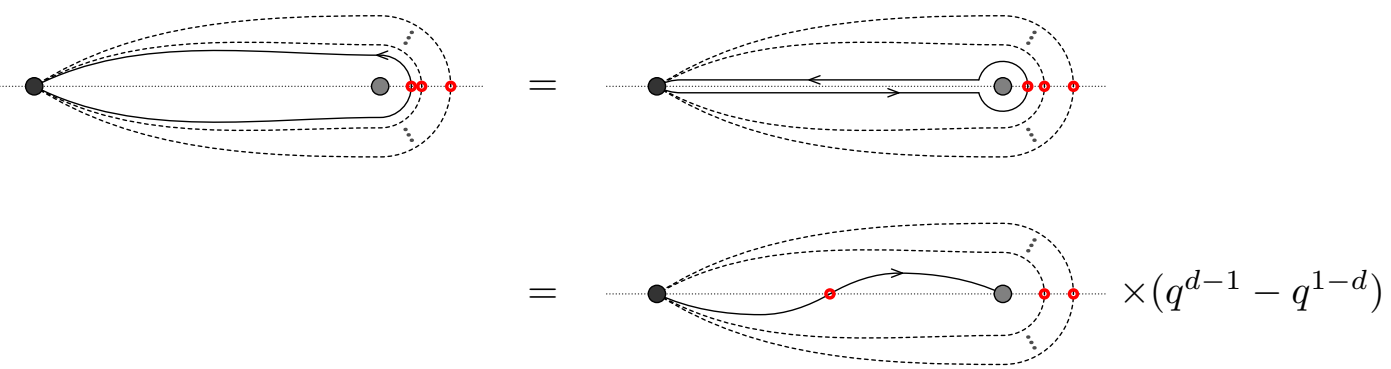

(b) First, the integration over the innermost loop $\mathcal{C}_{1}$ — corresponding to the variable $w_{1}$ — is rewritten according to the illustrations in this figure. The small circular arc can be neglected. Two pieces between $x_{0}$ and $x$ remain, and their contributions are the same up to orientation and phase factors.
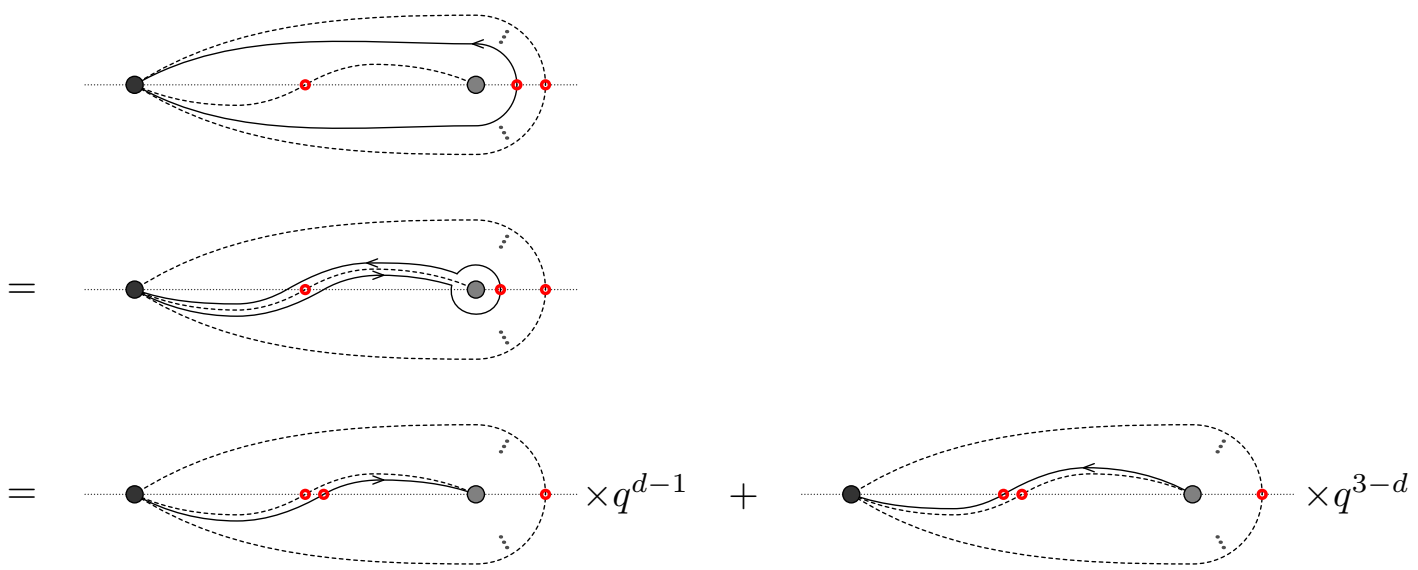

(c) Once the integration over $w_{1}$ has been rewritten as an integral from $x_{0}$ to $x$, we start manipulating the integration over the next loop $\mathcal{C}_{2}$ - corresponding to the variable $w_{2}$. The rewriting is illustrated in this figure. The result consists of two pieces, and upon relabeling the dummy integration variables $w_{1}$ and $w_{2}$ in one of them, the contributions are seen to be the same up to phase factors and signs.

Figure A.1. Illustrations for the proof of Lemma 3.5 . 
corresponding piece of the contour remains in the subset $\widetilde{\mathfrak{W}}_{\ell}$. The phase factor accumulated in this case is $q^{1-d+2(r-1)}$. After extracting all these phase factors, the remaining integral is, up to an orientation, equal to the integral $\widetilde{\rho}_{\ell}^{\left(x_{0}\right)}(x)$. The final result is

$$
\varphi_{\ell}^{\left(x_{0}\right)}(x)=\prod_{r=1}^{\ell}\left(q^{d-1}-q^{1-d+2(r-1)}\right) \times \int_{\widetilde{\mathcal{R}}_{\ell}} f_{\tilde{\ell}} \widetilde{(}\left(x ; w_{1}, \ldots, w_{\ell}\right) \mathrm{d} w_{1} \cdots \mathrm{d} w_{\ell},
$$

and we finish the proof by simplifying the prefactor

$$
\prod_{r=1}^{\ell}\left(q^{d-1}-q^{1-d+2(r-1)}\right)=q^{\left(\begin{array}{l}
\ell \\
2
\end{array}\right)} \prod_{m=1}^{\ell}\left(q^{d-m}-q^{m-d}\right)
$$

and using Lemma 3.2 If $\ell \geq d$, the product contains a factor which vanishes, and then $\varphi_{\ell}^{\left(x_{0}\right)}(x)=0$.

Lemma (Lemma 3.6). We have

$$
\begin{aligned}
\varphi_{l_{1}, l_{2}}^{\left(x_{0}\right)}\left(x_{1}, x_{2}\right)=q^{\left(\begin{array}{c}
l_{1} \\
2
\end{array}\right)+\left(\begin{array}{c}
l_{2} \\
2
\end{array}\right)\left(q-q^{-1}\right)^{l_{1}+l_{2}} \frac{\left[d_{1}-1\right] !\left[d_{2}-1\right] !}{\left[d_{1}-l_{1}-1\right] !\left[d_{2}-l_{2}-1\right] !}} \\
\times \sum_{m=0}^{l_{2}} q^{m\left(m-l_{2}+d_{1}-1\right)}\left[\begin{array}{c}
l_{2} \\
m
\end{array}\right] \widetilde{\rho}_{l_{1}+m, l_{2}-m}^{\left(x_{0}\right)}\left(x_{1}, x_{2}\right) .
\end{aligned}
$$

Proof. We use the method of the proof of Lemma 3.5 to decompose the $l_{1}$ loops around $x_{1}$ and the $l_{2}$ loops around $x_{2}$ to paths from $x_{0}$ to $x_{1}$ and $x_{2}$, respectively. We obtain

$$
\varphi_{l_{1}, l_{2}}^{\left(x_{0}\right)}\left(x_{1}, x_{2}\right)=\prod_{i=1,2}\left(q^{\left(\begin{array}{c}
l_{i} \\
2
\end{array}\right)} \prod_{t=1}^{l_{i}}\left(q^{d_{i}-t}-q^{t-d_{i}}\right)\right) \times \widetilde{\omega}_{l_{1}, l_{2}}^{\left(x_{0}\right)}\left(x_{1}, x_{2}\right),
$$

where $\widetilde{\omega}_{l_{1}, l_{2}}^{\left(x_{0}\right)}$ is the function defined by an integral as in Figure A.2. The variables $w_{1}, \ldots, w_{l_{1}}$ are integrated from $x_{0}$ to $x_{1}$ and the variables $w_{l_{1}+1}, \ldots, w_{l_{1}+l_{2}}$ from $x_{0}$ to $x_{2}$ in such a way that for all $r<r^{\prime}$, the path of the variable $w_{r^{\prime}}$ remains below the path of the variable $w_{r}$. The integrand is rephased so that it is positive in the region

$$
x_{0}<w_{1}<w_{2}<\cdots<w_{l_{1}}<x_{1}<w_{l_{1}+1}<\cdots<w_{l_{1}+l_{2}}<x_{2} .
$$

We further split the integration contours of the $l_{2}$ variables $w_{l_{1}+1}, \ldots, w_{l_{1}+l_{2}}$ into two pieces: the first from $x_{0}$ to $x_{1}$ and the second from $x_{1}$ to $x_{2}$. A contribution proportional to $\widetilde{\rho}_{l_{1}+m, l_{2}-m}^{\left(x_{0}\right)}$ is obtained whenever we make the first choice for some $m$ of the $l_{2}$ variables - we again just have to keep track of the correct phase factors. Suppose that we make the first choice for the variables

$$
w_{l_{1}+s_{1}}, w_{l_{1}+s_{2}}, \ldots, w_{l_{1}+s_{m}} \quad\left(1 \leq s_{1}<s_{2}<\cdots<s_{m} \leq l_{2}\right)
$$

and the second choice for the rest. Then, when taking the variables from the point where the integrand of $\widetilde{\omega}_{l_{1}, l_{2}}^{\left(x_{0}\right)}$ is positive to the point where the integrand of $\widetilde{\rho}_{l_{1}+m, l_{2}-m}^{\left(x_{0}\right)}$ is positive, we accumulate some phase. Indeed, first of all, each $w_{l_{1}+s_{p}}$ goes half a negative turn around $x_{1}$, contributing a factor $q^{d_{1}-1}$. Moreover, each $w_{l_{1}+s_{p}}$ goes half a negative turn around each $w_{r}$ with $l_{1}<r<l_{1}+s_{p}$, for $r \neq l_{1}+s_{p^{\prime}}$, contributing a phase factor $q^{-2\left(s_{p}-p\right)}$. In the end, all the contributions to $\widetilde{\omega}_{l_{1}, l_{2}}^{\left(x_{0}\right)}$ with fixed $m$ give

$$
q^{\left(d_{1}-1\right) m} \times \sum_{1 \leq s_{1}<s_{2}<\cdots<s_{m} \leq l_{2}} q^{-2 \sum_{p}\left(s_{p}-p\right)} \times \widetilde{\rho}_{l_{1}+m, l_{2}-m}^{\left(x_{0}\right)} .
$$

By Lemma 2.1 (c) we simplify the sum of the prefactors to the form $q^{-m\left(l_{2}-m\right)}\left[\begin{array}{l}l_{2} \\ m\end{array}\right]$, and obtain

$$
\widetilde{\omega}_{l_{1}, l_{2}}^{\left(x_{0}\right)}=\sum_{m=0}^{l_{2}} q^{\left(d_{1}-1+m-l_{2}\right) m}\left[\begin{array}{l}
l_{2} \\
m
\end{array}\right] \times \widetilde{\rho}_{l_{1}+m, l_{2}-m}^{\left(x_{0}\right)} .
$$




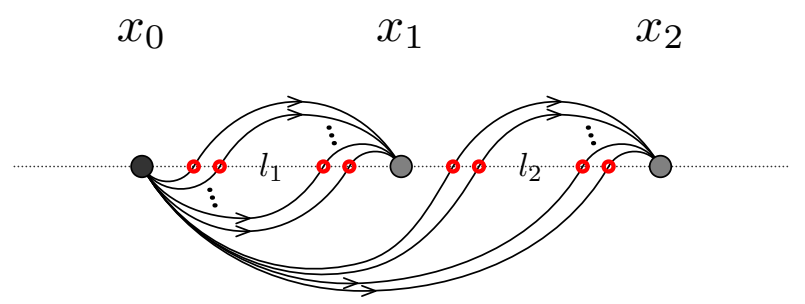

FIgURE A.2. The integration surface for the function $\widetilde{\omega}_{l_{1}, l_{2}}^{\left(x_{0}\right)}\left(x_{1}, x_{2}\right)$ used for the decomposition in the proof of Lemma 3.6. The integrand is rephased to be real and positive at the point $x_{0}<w_{1}<\cdots<w_{l_{1}}<x_{1}<w_{l_{1}+1}<\cdots<w_{l_{1}+l_{2}}<x_{2}$, marked by the red circles.

We finally write

$$
q^{\left(\begin{array}{c}
l_{i} \\
2
\end{array}\right)} \prod_{t=1}^{l_{i}}\left(q^{d_{i}-t}-q^{t-d_{i}}\right)=q^{\left(\begin{array}{c}
l_{i} \\
2
\end{array}\right)}\left(q-q^{-1}\right)^{l_{i}} \frac{\left[d_{i}-1\right] !}{\left[d_{i}-l_{i}-1\right] !},
$$

and the expression for $\varphi_{l_{1}, l_{2}}^{\left(x_{0}\right)}$ then takes the form

$$
\begin{aligned}
\varphi_{l_{1}, l_{2}}^{\left(x_{0}\right)}\left(x_{1}, x_{2}\right)=q^{\left(\begin{array}{c}
l_{1} \\
2
\end{array}\right)+\left(\begin{array}{c}
l_{2} \\
2
\end{array}\right)\left(q-q^{-1}\right)^{l_{1}+l_{2}} \frac{\left[d_{1}-1\right] !\left[d_{2}-1\right] !}{\left[d_{1}-l_{1}-1\right] !\left[d_{2}-l_{2}-1\right] !}} \\
\times \sum_{m=0}^{l_{2}} q^{\left(d_{1}-1+m-l_{2}\right) m}\left[\begin{array}{c}
l_{2} \\
m
\end{array}\right] \times \widetilde{\rho}_{l_{1}+m, l_{2}-m}^{\left(x_{0}\right)}\left(x_{1}, x_{2}\right) .
\end{aligned}
$$

This finishes the proof.

Lemma (Lemma 3.7). We have

$$
\varphi_{l_{1}, \ldots, l_{n}}^{\left(x_{0}\right)}(\boldsymbol{x})=\sum_{m_{1}, \ldots, m_{n}} C_{l_{1}, \ldots, l_{n}}^{m_{1}, \ldots, m_{n}} \times \widetilde{\rho}_{m_{1}, \ldots, m_{n}}^{\left(x_{0}\right)}(\boldsymbol{x}),
$$

with some coefficients $C_{l_{1}, \ldots, l_{n}}^{m_{1}, \ldots, m_{n}}$, which are zero unless $\sum_{i} l_{i}=\sum_{i} m_{i}$ and $\sum_{i=1}^{j} l_{i} \leq \sum_{i=1}^{j} m_{i}$ for all $j=1, \ldots, n$.

Proof. We only give a rough outline, and leave the details to the reader. By a generalization of the method used in the proof of Lemma 3.6, one can show that

$$
\begin{aligned}
& \varphi_{l_{1}, \ldots, l_{n}}^{\left(x_{0}\right)}=\prod_{i=1}^{n} q^{\left(\begin{array}{c}
l_{i} \\
2
\end{array}\right)} \prod_{t=1}^{l_{i}}\left(q^{d_{i}-t}-q^{t-d_{i}}\right) \\
& \times \sum_{\substack{\left(k_{j}^{(i)}\right) \\
\forall i \leq i \leq n ; 1 \leq j \leq i \\
\forall i: \sum_{j=1}^{i} k_{j}^{(i)}=l_{i}}} C_{l_{1}, \ldots, l_{n}}\left(\left(k_{j}^{(i)}\right)_{1 \leq i \leq n ; 1 \leq j \leq i}\right) \times \widetilde{\rho}_{\left.\sum_{i \geq 1} x_{0}\right)}^{(i)}, \sum_{i \geq 2} k_{2}^{(i)}, \ldots, k_{n}^{(n)},
\end{aligned}
$$

where

$$
\begin{aligned}
& C_{l_{1}, \ldots, l_{n}}\left(\left(k_{j}^{(i)}\right)_{1 \leq i \leq n ; 1 \leq j \leq i}\right) \\
= & \prod_{i}\left[\begin{array}{c}
l_{i} \\
k_{1}^{(i)} ; k_{2}^{(i)} ; \cdots ; k_{i}^{(i)}
\end{array}\right] q^{-\sum_{j^{\prime}<j} k_{j}^{(i)} k_{j^{\prime}}^{(i)}} \prod_{i<i^{\prime}} q^{-2 \sum_{j^{\prime}<j} k_{j}^{(i)} k_{j^{\prime}}^{\left(i^{\prime}\right)}} \prod_{i<i^{\prime}} q^{\sum_{j \leq i}\left(d_{i}-1\right) k_{j}^{\left(i^{\prime}\right)}},
\end{aligned}
$$


and where we have used the following $q$-multinomial coefficients

$$
\left[\begin{array}{c}
\ell \\
k_{1} ; k_{2} ; \cdots ; k_{i}
\end{array}\right]=\frac{[\ell] !}{\prod_{j=1}^{i}\left[k_{j}\right] !}
$$

The desired coefficients of $\widetilde{\rho}_{m_{1}, \ldots, m_{n}}$ are then expressible as sums of the coefficients above,

$$
C_{l_{1}, \ldots, l_{n}}^{m_{1}, \ldots, m_{n}}=\prod_{i=1}^{n} q^{\left(\begin{array}{c}
l_{i} \\
2
\end{array}\right)} \prod_{t=1}^{l_{i}}\left(q^{d_{i}-t}-q^{t-d_{i}}\right) \times \sum_{\substack{\left(k_{j}^{(i)}\right) \\
\forall j: \sum_{i \geq j} k_{j}^{(i)}=m_{j}}} C_{l_{1}, \ldots, l_{n}}\left(\left(k_{j}^{(i)}\right)_{1 \leq i \leq n ; 1 \leq j \leq i}\right) .
$$

Lemma (Lemma 3.8). Whenever $l_{i} \geq d_{i}$ for some $i=1, \ldots, n$, we have

$$
\varphi_{l_{1}, \ldots, l_{n}}^{\left(x_{0}\right)}\left(x_{1}, \ldots, x_{n}\right) \equiv 0 .
$$

Proof. We may rearrange the integrations over those $l_{i} w$-variables which encircle $x_{i}$ similarly as in the case of Lemma 3.5. After this rearrangement, the result of the whole integral $\varphi_{l_{1}, \ldots, l_{n}}^{\left(x_{0}\right)}(\boldsymbol{x})$ is the factor $\left[\ell_{i}\right] ! \prod_{m=1}^{l_{i}}\left(q^{d_{i}-m}-q^{m-d_{i}}\right)$ times an integral which is convergent for large $\kappa$. The result thus again vanishes if $l_{i} \geq d_{i}$ and $\kappa$ is large, and by analyticity in $\kappa$, the same conclusion is valid for all values of $\kappa>0$.

\section{Appendix B. Differential operators ACting on the Integrand}

In this appendix, we outline the proof of Lemma 4.9, which is used to show, roughly speaking, that the differential operators acting on our integrands produce exact forms.

Lemma (Lemma 4.9p. Let $d_{1}, \ldots, d_{n}$ be real numbers. The function

$$
f^{(0)}\left(x_{1}, \ldots, x_{n}\right)=\prod_{1 \leq i<j \leq n}\left(x_{j}-x_{i}\right)^{\frac{2}{\kappa}\left(d_{i}-1\right)\left(d_{j}-1\right)}
$$

satisfies the partial differential equation $\mathcal{D}_{d_{j}}^{(j)} f^{(0)}=0$, for all $j=1, \ldots, n$ such that $d_{j}$ is a positive integer.

Proof. If $d_{j}=1$, the claim $\mathcal{D}_{1}^{(j)} f^{(0)}\left(x_{1}, \ldots, x_{n}\right)=\frac{\partial}{\partial x_{j}} f\left(x_{1}, \ldots, x_{n}\right)=0$ is obvious: the function is constant in the variable $x_{j}$.

In the case $d_{j}=2$, the claim $\mathcal{D}_{2}^{(j)} f^{(0)}\left(x_{1}, \ldots, x_{n}\right)=0$ has been verified by an explicit calculation in [Kyt07, Lemma 5.1]. We take this special case as our starting point, and proceed by a recursive argument, which employs fusion similar to [Dub15, Theorem 15].

We perform an induction on the number

$$
D=\sum_{\substack{j \\ d_{j} \in\{3,4, \ldots\}}}\left(d_{j}-2\right) \in \mathbb{Z}_{\geq 0} .
$$

The base case $D=0$ is covered by the initial observations above. In the induction step, we want to increment $D$ by one, which amounts to being able to increment one of the parameters that are integers greater than or equal to 2 . By permutation symmetry, we may assume that $d_{1}=d$ is the parameter we wish to increment. We may also take $d_{2}=2$, which will allow us to get to $d+1$ by fusion.

Hence, fix parameters $d_{1}, \ldots, d_{n}$ such that

$$
d_{1}=d \in\{2,3, \ldots\} \quad \text { and } \quad d_{2}=2
$$


and assume that the claim has been verified for the corresponding $f^{(0)}$. For notational convenience, we will denote the two first variables by $x_{1}=z$ and $x_{2}=y$. The fusion is based on the following obvious asymptotics of $f^{(0)}$. If we denote

$$
\Delta=h_{1, d+1}-h_{1, d}-h_{1,2}=\frac{2}{\kappa}(d-1),
$$

then the function defined by

$$
g_{0}\left(z, x_{3}, \ldots, x_{n}\right)=\lim _{y \rightarrow z} \frac{1}{(z-y)^{\Delta}} f^{(0)}\left(z, y, x_{3}, \ldots, x_{n}\right)
$$

is of the same type as $f^{(0)}$, but with the new parameter sequence $d+1, d_{3}, \ldots, d_{n}$, simply because

$$
\left(x_{j}-z\right)^{\frac{2}{\kappa}(d-1)\left(d_{j}-1\right)}\left(x_{j}-y\right)^{\frac{2}{\kappa}\left(d_{j}-1\right)} \underset{y \rightarrow z}{\longrightarrow}\left(x_{j}-z\right)^{\frac{2}{\kappa} d\left(d_{j}-1\right)} .
$$

Our goal is to verify the asserted partial differential equations for this $g_{0}$, assuming the ones on $f^{(0)}$. The most involved among them is the partial differential equation of order $d+1$ given by

$$
\mathcal{D}_{d+1} g_{0}\left(z, x_{3}, \ldots, x_{n}\right)=0,
$$

where

$$
\mathcal{D}_{d+1}=\sum_{k=1}^{d+1} \sum_{\substack{p_{1}, \ldots, p_{k} \geq 1 \\ p_{1}+\cdots+p_{k}=d+1}} \frac{(-4 / \kappa)^{d+1-k} d !^{2}}{\prod_{u=1}^{k-1}\left(\sum_{i=1}^{u} p_{i}\right)\left(\sum_{i=u+1}^{k} p_{i}\right)} \times \mathcal{L}_{-p_{1}} \cdots \mathcal{L}_{-p_{k}}
$$

and

$$
\mathcal{L}_{-p}=-\sum_{i=3}^{n}\left(x_{i}-z\right)^{1-p} \frac{\partial}{\partial x_{i}}-(1-p) \sum_{i=3}^{n}\left(x_{i}-z\right)^{-p} h_{1, d_{i}} .
$$

In order to verify (B.2), we use the induction assumption that $f^{(0)}$ satisfies, in particular, the following two differential equations respectively associated to the points $z$ and $y$ :

$$
\begin{aligned}
& \widehat{\mathcal{D}}_{d} f^{(0)}\left(z, y, x_{3}, \ldots, x_{n}\right)=0 \\
& \widetilde{\mathcal{D}}_{2} f^{(0)}\left(z, y, x_{3}, \ldots, x_{n}\right)=0,
\end{aligned}
$$

where

$$
\begin{aligned}
& \widehat{\mathcal{D}}_{d}=\sum_{k=1}^{d} \sum_{\substack{p_{1}, \ldots, p_{k} \geq 1 \\
p_{1}+\cdots+p_{k}=d}} \frac{(-4 / \kappa)^{d-k}(d-1) !^{2}}{\prod_{u=1}^{k-1}\left(\sum_{i=1}^{u} p_{i}\right)\left(\sum_{i=u+1}^{k} p_{i}\right)} \times \widehat{\mathcal{L}}_{-p_{1}} \cdots \widehat{\mathcal{L}}_{-p_{k}} \\
& \widetilde{\mathcal{D}}_{2}=\widetilde{\mathcal{L}}_{-1}^{2}-\frac{4}{\kappa} \widetilde{\mathcal{L}}_{-2},
\end{aligned}
$$

with

$$
\begin{aligned}
& \widehat{\mathcal{L}}_{-p}=-(y-z)^{1-p} \frac{\partial}{\partial y}-(1-p)(y-z)^{-p} h_{1,2}-\sum_{i=3}^{n}\left(x_{i}-z\right)^{1-p} \frac{\partial}{\partial x_{i}}-(1-p) \sum_{i=3}^{n}\left(x_{i}-z\right)^{-p} h_{1, d_{i}} \\
& \widetilde{\mathcal{L}}_{-p}=-(z-y)^{1-p} \frac{\partial}{\partial z}-(1-p)(z-y)^{-p} h_{1, d}-\sum_{i=3}^{n}\left(x_{i}-y\right)^{1-p} \frac{\partial}{\partial x_{i}}-(1-p) \sum_{i=3}^{n}\left(x_{i}-y\right)^{-p} h_{1, d_{i}} .
\end{aligned}
$$

To study the limit function $g_{0}$ defined by (B.1), we perform an expansion in $\varepsilon=y-z$ around $\varepsilon=0$. We thus write first of all

$$
f^{(0)}\left(z, y, x_{3}, \ldots, x_{n}\right)=\sum_{m=0}^{\infty} \varepsilon^{\Delta+m} g_{m}\left(z, x_{3}, \ldots, x_{n}\right) .
$$


Moreover, we write

$$
\widehat{\mathcal{L}}_{-p}=-\varepsilon^{1-p} \frac{\partial}{\partial \varepsilon}-(1-p) \varepsilon^{-p} h_{1,2}+\mathcal{L}_{-p},
$$

and note that the action of $\widetilde{\mathcal{L}}_{-1}$ and $\widetilde{\mathcal{L}}_{-2}$ on the translation invariant function $f^{(0)}$ is

$$
\widehat{\mathcal{L}}_{-1}=\frac{\partial}{\partial \varepsilon} \quad \text { and } \quad \widehat{\mathcal{L}}_{-2}=\sum_{s=-1}^{\infty} \varepsilon^{s} \mathcal{L}_{-2-s}-\varepsilon^{-1} \frac{\partial}{\partial \varepsilon}+\varepsilon^{-2} h_{1, d}
$$

With these, it is straightforward to calculate $\widetilde{\mathcal{D}}_{2} f^{(0)}$ in a series expansion in $\varepsilon$, and after some simplifications the result is

$$
\widetilde{\mathcal{D}}_{2} f^{(0)}=\sum_{m=0}^{\infty} \varepsilon^{\Delta+m-2}\left(r(\Delta+m) g_{m}-\frac{4}{\kappa} \sum_{p^{\prime}=1}^{m} \mathcal{L}_{-p^{\prime}} g_{m-p^{\prime}}\right),
$$

where $r(\alpha):=\alpha^{2}-\alpha+\frac{4}{\kappa} \alpha-\frac{4}{\kappa} h_{1, d}$. According to equation B.4 , this series must vanish term by term. Since also $r(\Delta+m) \neq 0$ for all $m=1,2, \ldots$, we can derive that

$$
g_{m}=\mathcal{R}_{m} g_{0},
$$

where

$$
\mathcal{R}_{m}=\sum_{u=1}^{m} \sum_{\substack{p_{1}^{\prime}, \ldots, p_{u}^{\prime} \geq 1 \\ p_{1}^{\prime}+\cdots+p_{k}^{\prime}=m}} \frac{(4 / \kappa)^{u}}{\prod_{j=1}^{u} r\left(\Delta+\sum_{i=j}^{u} p_{i}^{\prime}\right)} \mathcal{L}_{-p_{1}^{\prime}} \cdots \mathcal{L}_{-p_{u}^{\prime}} g_{0}
$$

The next step is to study the expression $\widehat{D}_{d} f^{(0)}$, as a series expanded in $\varepsilon$. Using the expression derived above, we get

$$
\widehat{\mathcal{D}}_{d} f^{(0)}=\sum_{m=0}^{\infty} \widehat{\mathcal{D}}_{d}\left(\varepsilon^{\Delta+m}\left(\mathcal{R}_{m} g_{0}\right)\right)=\sum_{m=0}^{\infty}\left(\mathcal{P}_{m} g_{0}\right) \varepsilon^{\Delta-d+m}
$$

where $\mathcal{P}_{m}$ is a polynomial in the operators $\mathcal{L}_{-p}$, for $p=1, \ldots$. By assumption $(\mathrm{B} .5)$, the series above must vanish term by term, i.e., $\mathcal{P}_{m} g_{0}=0$ for all $m$. From the argument in the proof of Dub15, Lemma 1] it follows that the polynomial $\mathcal{P}_{d+1}$ giving the coefficient of $\varepsilon^{\Delta+1}$ is a non-zero multiple of the differential operator $\mathcal{D}_{d+1}$ defined by (B.3). We conclude that $g_{0}$ satisfies the differential equation (B.2) of order $d+1$.

The other asserted differential equations for $g_{0}$ are easier to verify. The key is to still use the expansion $\left(\right.$ B.6) in $\varepsilon$, and note that in the first order differential operators $\mathcal{L}_{-p}^{(j)}$ acting on such an expansion, the following terms combine

$$
\begin{aligned}
& \left\{\left(z-x_{j}\right)^{1-p} \frac{\partial}{\partial z}+(1-p)\left(z-x_{j}\right)^{-p} h_{1, d}+\left(y-x_{j}\right)^{1-p} \frac{\partial}{\partial y}+(1-p)\left(y-x_{j}\right)^{-p} h_{1,2}\right\} \sum_{m=0}^{\infty} \varepsilon^{\Delta+m} g_{m} \\
= & \varepsilon^{\Delta} \times\left(\left(z-x_{j}\right)^{1-p} \frac{\partial}{\partial z}+(1-p)\left(z-x_{j}\right)^{-p}\left(h_{1, d}+h_{1,2}+\Delta\right)\right) g_{0}+\mathcal{O}\left(\varepsilon^{\Delta+1}\right) .
\end{aligned}
$$

Moreover, here we have $h_{1, d}+h_{1,2}+\Delta=h_{1, d+1}$. With these observations, it is routine to check that from the induction assumption that $f^{(0)}$ satisfies $\mathcal{D}_{d_{j}}^{(j)} f^{(0)}=0$ it follows that $g_{0}$ satisfies the corresponding partial differential equation of order $d_{j}$. This finishes the proof. 


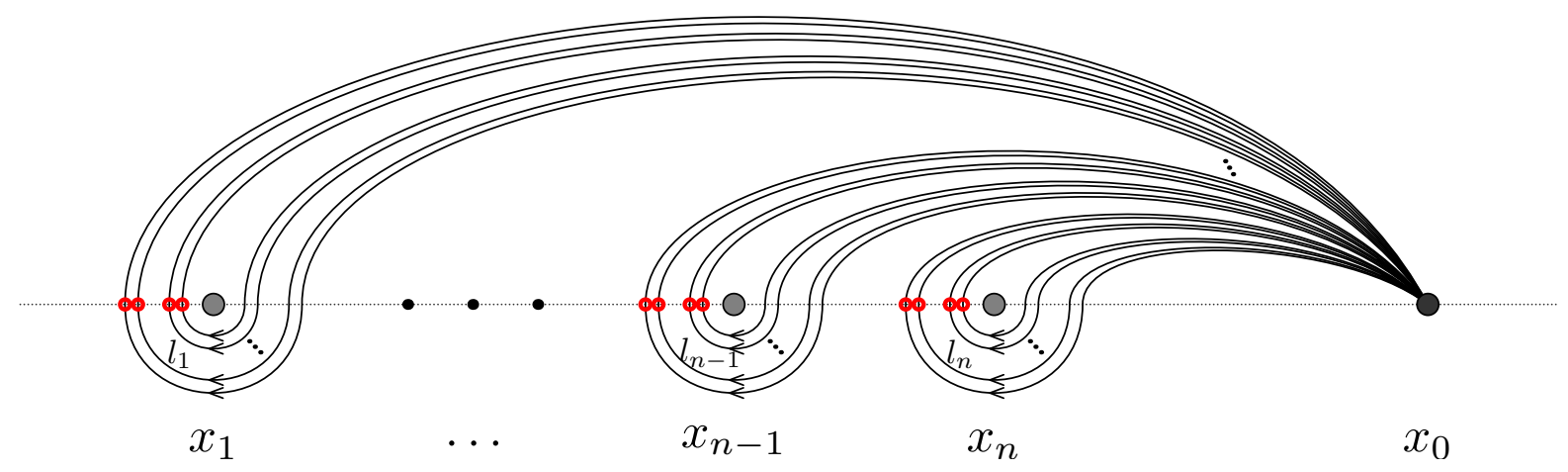

Figure C.1. The integration surface $\mathfrak{L}_{l_{1}, \ldots, l_{n}}^{\Subset}$. The point where the integrand $f_{l_{1}, \ldots, l_{n}}^{\Subset}(\boldsymbol{x} ; \cdot)$ is rephased to be positive is marked by red circles.

\section{Appendix C. Alternative conventions}

The main topic of the present article was the construction of the spin chain - Coulomb gas correspondence maps

$$
\mathcal{F}^{\left(x_{0}\right)}: \mathrm{M}_{d_{n}} \otimes \cdots \otimes \mathrm{M}_{d_{1}} \rightarrow \mathcal{C}^{\infty}\left(\mathfrak{X}_{n}^{\left(x_{0}\right)}\right),
$$

for which the representation theoretic properties on the quantum group side translate to properties of the functions. Our construction of the maps $\mathcal{F}^{\left(x_{0}\right)}$ necessarily involved certain somewhat arbitrary choices, and occasionally some other conventions could be considered more natural. In particular, it may be desirable to have the order of the tensorands correspond to the order of the variables $x_{1}<\cdots<x_{n}$ of the functions on the real line (the boundary of the upper half-plane), rather than to have the tensor product constructed in the reverse order. This appendix gives two alternatives to our conventions, which can be used to achieve the more intuitive order of tensor products. The first straightforward option is to modify the coproduct, which defines the tensor product representations for the quantum group. The second option is to modify the choice of basis functions and the restricted chamber in which the basis functions are defined. The first option alters the Hopf algebra structure of $\mathcal{U}_{q}\left(\mathfrak{s l}_{2}\right)$, and we will not explicitly list all the necessary changes in the representation theoretic lemmas. For the second choice, the Hopf algebra structure remains the same, so we can concisely state the corresponding versions of our main results for that case.

C.1. Opposite coproduct. The coproduct in Section 2.2.1 was chosen according to what appears more commonly in the quantum group literature. The algebra $\mathcal{U}_{q}\left(\mathfrak{s l}_{2}\right)$ could alternatively be equipped with a unique Hopf algebra structure corresponding to the coproduct

$$
\Delta^{\mathrm{op}}: \mathcal{U}_{q}\left(\mathfrak{s l}_{2}\right) \rightarrow \mathcal{U}_{q}\left(\mathfrak{s l}_{2}\right) \otimes \mathcal{U}_{q}\left(\mathfrak{s l}_{2}\right)
$$

given on the generators by the expressions

$$
\Delta^{\mathrm{op}}(E)=K \otimes E+E \otimes 1, \quad \Delta^{\mathrm{op}}(K)=K \otimes K, \quad \Delta^{\mathrm{op}}(F)=1 \otimes F+F \otimes K^{-1},
$$

i.e., with the order opposite to that of Section 2.2.1. It is then obvious that for the correspondence defined by

$$
\begin{gathered}
\mathcal{F}_{\mathrm{op}}^{\left(x_{0}\right)}: \mathrm{M}_{d_{1}} \otimes \cdots \otimes \mathrm{M}_{d_{n}} \rightarrow \mathcal{C}^{\infty}\left(\mathfrak{X}_{n}^{\left(x_{0}\right)}\right) \\
\mathcal{F}_{\mathrm{op}}^{\left(x_{0}\right)}\left[e_{l_{1}} \otimes \cdots \otimes e_{l_{n}}\right]=\varphi_{l_{1}, \ldots, l_{n}}^{\left(x_{0}\right)}
\end{gathered}
$$

analogues of our main results still hold. 


\section{C.2. Different basis functions.}

We finally present a way of keeping the standard coproduct while achieving the more natural order of tensor products. The Hopf algebra structure of $\mathcal{U}_{q}\left(\mathfrak{s l}_{2}\right)$ will thus be the same as elsewhere in the article, and all representation theoretic formulas of Section 2 hold without any changes. What we change now is the basis functions of the spin chain - Coulomb gas correspondence.

We take the anchor point $x_{0}$ for the new basis functions to lie to the right of all other variables, so the basis functions are defined on the chamber restricted from the right

$$
\overleftarrow{\mathfrak{X}}_{n}^{\left(x_{0}\right)}:=\left\{\left(x_{1}, \ldots, x_{n}\right) \in \mathbb{R}^{n} \mid x_{1}<\cdots<x_{n}<x_{0}\right\}
$$

Fix again dimension parameters $d_{1}, \ldots, d_{n} \in \mathbb{Z}_{>0}$. Analogously to the definition of basis functions in Section 3.3 , define now, for $l_{1}, \ldots, l_{n} \in \mathbb{Z}_{\geq 0}$, the function

$$
\overleftarrow{\varphi}_{l_{1}, \ldots, l_{n}}^{\left(x_{0}\right)}: \overleftarrow{\mathfrak{X}}_{n}^{\left(x_{0}\right)} \rightarrow \mathbb{C}
$$

as an integral over the surface $\mathfrak{L}_{l_{1}, \ldots, l_{n}}^{\Subset}$ depicted in Figure C.1. with the integrand $f_{l_{1}, \ldots, l_{n}}^{\Subset}$ rephased to be positive at the point marked in the figure,

$$
\overleftarrow{\varphi}_{l_{1}, \ldots, l_{n}}^{\left(x_{0}\right)}\left(x_{1}, \ldots, x_{n}\right)=\int_{\mathfrak{L}_{l_{1}, \ldots, l_{n}}^{\Subset}} f_{l_{1}, \ldots, l_{n}}^{\Subset}\left(x_{1}, \ldots, x_{n} ; w_{1}, \ldots, w_{\ell}\right) \mathrm{d} w_{1} \cdots \mathrm{d} w_{\ell}
$$

where $\ell=\sum_{j=1}^{n} l_{j}$ as before.

Then form the tensor product representation

$$
\mathrm{M}_{d_{1}} \otimes \cdots \otimes \mathrm{M}_{d_{n}}
$$

in the order that is opposite to our convention 4.1) used elsewhere. We define

$$
\overleftarrow{\mathcal{F}}_{d_{1}, \ldots, d_{n}}^{\left(x_{0}\right)}: \mathrm{M}_{d_{1}} \otimes \cdots \otimes \mathrm{M}_{d_{n}} \rightarrow \mathcal{C}^{\infty}\left(\overleftarrow{\mathfrak{X}}_{n}^{\left(x_{0}\right)}\right)
$$

by setting

$$
\overleftarrow{\mathcal{F}}_{d_{1}, \ldots, d_{n}}^{\left(x_{0}\right)}\left[e_{l_{1}}^{\left(d_{1}\right)} \otimes \cdots \otimes e_{l_{n}}^{\left(d_{n}\right)}\right]=\overleftarrow{\varphi}_{l_{1}, \ldots, l_{n}}^{\left(x_{0}\right)}
$$

and extending linearly. Below we again omit the subscript dimensions from the notation. This alternative correspondence $\overleftarrow{\mathcal{F}}$ has properties entirely similar to $\mathcal{F}$. In particular, for $v \in \mathrm{M}_{d_{1}} \otimes \cdots \otimes \mathrm{M}_{d_{n}}$ we have:

- (well-def.): If $E \cdot v=0$, then $\overleftarrow{\mathcal{F}}^{\left(x_{0}\right)}[v]\left(x_{1}, \ldots, x_{n}\right)$ is independent of $x_{0}$, and thus defines a function

$$
\overleftarrow{\mathcal{F}}[v]: \mathfrak{X}_{n} \rightarrow \mathbb{C}
$$

- (COV): For any $\xi \in \mathbb{R}$ we have the translation invariance

$$
\overleftarrow{\mathcal{F}}^{\left(x_{0}+\xi\right)}[v]\left(x_{1}+\xi, \ldots, x_{n}+\xi\right)=\overleftarrow{\mathcal{F}}^{\left(x_{0}\right)}[v]\left(x_{1}, \ldots, x_{n}\right)
$$

If furthermore $K \cdot v=q^{d-1} v$, then for any $\lambda>0$ we have the scaling covariance

$$
\overleftarrow{\mathcal{F}}^{\left(\lambda x_{0}\right)}[v]\left(\lambda x_{1}, \ldots, \lambda x_{n}\right)=\lambda^{\Delta_{d}^{d_{1}, \ldots, d_{n}}} \times \overleftarrow{\mathcal{F}}^{\left(x_{0}\right)}[v]\left(x_{1}, \ldots, x_{n}\right)
$$

If $K \cdot v=v$ and $E \cdot v=0$, then we have the full Möbius covariance

$$
\prod_{j=1}^{n} \mu^{\prime}\left(x_{j}\right)^{h_{1, d_{j}}} \times \overleftarrow{\mathcal{F}}[v]\left(\mu\left(x_{1}\right), \ldots, \mu\left(x_{n}\right)\right)=\overleftarrow{\mathcal{F}}[v]\left(x_{1}, \ldots, x_{n}\right)
$$

for any Möbius transformation $\mu: \mathbb{H} \rightarrow \mathbb{H}$ such that $\mu\left(x_{1}\right)<\mu\left(x_{2}\right)<\cdots<\mu\left(x_{n}\right)$.

- (PDE): If $E \cdot v=0$, we have

$$
\mathcal{D}_{d_{j}}^{(j)} \overleftarrow{\mathcal{F}}[v]=0 \quad \text { for } j=1, \ldots, n
$$

where $\mathcal{D}_{d_{j}}^{(j)}$ is the differential operator 4.12 . 
- (ASY): Suppose that $v$ belongs to the subrepresentation obtained by picking the $d$-dimensional irreducible direct summand in the tensor product of the $j$ :th and $j+1$ :st factors $\mathrm{M}_{d_{j}}$ and $\mathrm{M}_{d_{j+1}}$ (now counting from the left), and denote by

$$
\hat{v} \in\left(\mathrm{M}_{d_{1}} \otimes \cdots \otimes \mathrm{M}_{d_{j-1}}\right) \otimes \mathrm{M}_{d} \otimes\left(\mathrm{M}_{d_{j+2}} \otimes \cdots \otimes \mathrm{M}_{d_{n}}\right)
$$

the vector obtained by identifying $v$ as a vector in an $(n-1)$-fold tensor product representation. More precisely, with the earlier notations, this means $v=\pi_{N-j, N-j+1}^{(d)}(v)$ and $\hat{v}=\hat{\pi}_{N-j, N-j+1}^{(d)}(v)$. Then we have

$$
\begin{aligned}
\lim _{x_{j}, x_{j+1} \rightarrow \xi} & \left(\left(x_{j+1}-x_{j}\right)^{-\Delta_{d}^{d_{j}, d_{j+1}}} \times \overleftarrow{\mathcal{F}}^{\left(x_{0}\right)}[v]\left(x_{1}, \ldots, x_{n}\right)\right) \\
= & B_{d}^{d_{j}, d_{j+1}} \times \overleftarrow{\mathcal{F}}^{\left(x_{0}\right)}[\hat{v}]\left(x_{1}, \ldots, x_{j-1}, \xi, x_{j+2}, \ldots, x_{n}\right)
\end{aligned}
$$

Also the further results of Section $\sqrt[5]{5}$ have straightforward analogues for $\overleftarrow{\mathcal{F}}$

\section{REFERENCES}

[AKL12] T. Alberts, M. J. Kozdron, and G. F. Lawler. The Green's function for the radial Schramm-Loewner evolution. J. Phys. A: Math. Theor., 45:494015, 2012.

[BBK05] M. Bauer, D. Bernard, and K. Kytölä. Multiple Schramm-Loewner evolutions and statistical mechanics martingales. J. Stat. Phys., 120(5-6):1125-1163, 2005.

[BFGT09] P. V. Bushlanov, B. L. Fergin, A. M. Gainutdinov, and I. Yu. Tipunin. Lusztig limit of quantum sl(2) at root of unity and fusion of (1,p) Virasoro logarithmic minimal models. Nucl. Phys. B, 818(3):179-195, 2009.

[BMP90] P. Bouwknegt, J. McCarthy, and K. Pilch. Quantum group structure in the Fock space resolutions of $\hat{s l}(n)$ representations. Comm. Math. Phys., 131(1):125-155, 1990.

[BSA88] L. Benoit and Y. Saint-Aubin. Degenerate conformal field theories and explicit expressions for some null vectors. Phys. Lett., B215(3):517-522, 1988.

[BPZ84] A. A. Belavin, A. M. Polyakov, and A. B. Zamolodchikov. Infinite conformal symmetry in two-dimensional quantum field theory. Nucl. Phys. B 241(2):333-380, 1984.

$\left[\mathrm{CDCH}^{+}{ }^{14}\right]$ D. Chelkak, H. Duminil-Copin, C. Hongler, A. Kemppainen, and S. Smirnov. Convergence of Ising interfaces to Schramm's SLE curves. C. R. Acad. Sci. Paris Sér. I Math., 352(2):157-161, 2014.

[CN07] F. Camia and C. M. Newman. Critical percolation exploration path and $\mathrm{SLE}_{6}$ : a proof of convergence. Probab. Theory Related Fields, 139(3-4):473-519, 2007.

[CS12] D. Chelkak and S. Smirnov. Universality in the 2D Ising model and conformal invariance of fermionic observables. Invent. Math., 189(3):515-580, 2012.

[DF84] V. S. Dotsenko and V. A. Fateev. Conformal algebra and multipoint correlation functions in 2D statistical models. Nucl. Phys., B240(3):312-348, 1984.

[DF85] V. S. Dotsenko and V. A. Fateev. Four-point correlation functions and the operator algebra in 2D conformal invariant theories with $c \geq 1$. Nucl. Phys., B251:691-734, 1985.

[Dub06] J. Dubédat. Euler integrals for commuting SLEs. J. Stat. Phys., 123(6):1183-1218, 2006.

[Dub07] J. Dubédat. Commutation relations for SLE. Comm. Pure Appl. Math., 60(12):1792-1847, 2007.

[Dub15] J. Dubédat. SLE and Virasoro representations: Fusion. Comm. Math. Phys., 336(2):761-809, 2015.

[Fel89] G. Felder. BRST approach to minimal models. Nucl. Phys. B, 317(1):215-236, 1989. Erratum ibid. B, 324(2):548, 1989.

[FF90] B. L. Fergin and D. B. Fuchs. Representations of the Virasoro algebra. In Representation of Lie groups and related topics, volume 7 of Adv. Stud. Contemp. Math., pages 465-554. Gordon and Breach, New York, 1990.

[FFK89] G. Felder, J. Fröhlich, and G. Keller. Braid matrices and structure constants for minimal conformal models. Comm. Math. Phys., 124(4):647-664, 1989.

[FK15a] S. M. Flores and P. Kleban. A solution space for a system of null-state partial differential equations, Part I. Comm. Math. Phys., 333(1):389-434, 2015.

[FK15b] S. M. Flores and P. Kleban. A solution space for a system of null-state partial differential equations, Part II. Comm. Math. Phys., 333(1):435-481, 2015.

[FK15c] S. M. Flores and P. Kleban. A solution space for a system of null-state partial differential equations, Part III. Comm. Math. Phys., 333(2):597-667, 2015.

[FK15d] S. M. Flores and P. Kleban. A solution space for a system of null-state partial differential equations, Part IV. Comm. Math. Phys., 333(2):669-715, 2015.

[For10] P. Forrester. Log-gases and random matrices. London Mathematical Society Monographs 34, Princeton Univ. Press, 2010. 
[FP18a] S. M. Flores and E. Peltola. Higher quantum and classical Schur-Weyl duality for $\mathfrak{s l}_{2}(\mathbb{C})$. In preparation, 2018.

[FP18b] S. M. Flores and E. Peltola. Monodromy invariant CFT correlation functions of first column Kac operators. In preparation, 2018.

[FSK15] S. M. Flores, J. J. H. Simmons, and P. Kleban. Multiple-SLE $\kappa_{\kappa}$ connectivity weights for rectangles, hexagons, and octagons. Preprint: http://arxiv.org/abs/1505.07756 2015.

[FSKZ17] S. M. Flores, J. J. H. Simmons, P. Kleban, and R. M. Ziff. A formula for crossing probabilities of critical systems inside polygons. J. Phys. A: Math. Theor., 50(6):064005, 2017.

[Fuc92] J. Fuchs. Affine Lie algebras and quantum groups. Cambridge Monographs on Mathematical Physics. Cambridge Univ. Press, 1992.

[FW91] G. Felder and C. Wieczerkowski. Topological representation of the quantum group $\mathcal{U}_{q}\left(\mathfrak{s l}_{2}\right)$. Comm. Math. Phys., 138(3):583-605, 1991.

[GRAS96] C. Gómez, M. Ruiz-Altaba, and G. Sierra. Quantum groups in two-dimensional physics. Cambridge Univ. Press, 1996.

[Gra07] K. Graham. On multiple Schramm-Loewner evolutions. J. Stat. Mech.: Theory and Exp., P03008, 2007.

[GS90] C. Gómez and G. Sierra. Quantum group meaning of the Coulomb gas. Phys. Lett., 240B(1-2):149-157, 1990.

[IK11] K. Iohara and Y. Koga. Representation theory of the Virasoro algebra. Springer Monographs in Mathematics. Springer, 2011.

[Izy11] K. Izyurov. Holomorphic spinor observables and interfaces in the critical Ising model. Ph.D. thesis, Université de Genève, 2011.

[JJK16] N. Jokela, M. Järvinen, and K. Kytölä. SLE boundary visits. Ann. Henri Poincaré 17(6):1263-1330, 2016.

[KL07] M. J. Kozdron and G. F. Lawler. The configurational measure on mutually avoiding SLE paths. In Universality and Renormalization: From Stochastic Evolution to Renormalization of Quantum Fields, Fields Inst. Commun. Amer. Math. Soc., 2007.

[KP16] K. Kytölä and E. Peltola. Pure partition functions of multiple SLEs. Comm. Math. Phys. 346(1):237-292, 2016.

[KS18] A. Kemppainen and S. Smirnov. Configurations of FK Ising interfaces and hypergeometric SLE. Math. Res. Lett., to appear, 2018. Preprint: http://arxiv.org/abs/1704.02823.

[Kyt07] K. Kytölä. Virasoro module structure of local martingales of SLE variants. Rev. Math. Phys., 19(5):455-509, 2007.

[Law15] G. F. Lawler. Minkowski content of the intersection of a Schramm-Loewner Evolution (SLE) curve with the real line. J. Math. Soc. Japan 67(4):1631-1669, 2015.

[LSW04] G. F. Lawler, O. Schramm, and W. Werner. Conformal invariance of planar loop-erased random walks and uniform spanning trees. Ann. Probab., 32(1B):939-995, 2004.

[LS11] G. F. Lawler and S. Sheffield. A natural parametrization for the Schramm-Loewner evolution. Ann. Probab., 39(5):1896-1937, 2011.

[LW13] G. F. Lawler and B. M. Werness. Multi-point Green's functions for SLE and an estimate of Beffara. Ann. Probab., 41(3A):1513-1555, 2013.

[LZ13] G. F. Lawler and W. Zhou. SLE curves and natural parametrization. Ann. Probab., 41(3A):1556-1584, 2013.

[MR89] G. Moore and N. Reshetikhin. A comment on quantum group symmetry in conformal field theory. Nucl. Phys., B328(3):557-574, 1989.

[PS91] V. Pasquier and H. Saleur. Common structures between finite systems and conformal field theories through quantum groups. Nucl. Phys., B330(2):523-556, 1990.

[Pel18] E. Peltola. Basis for solutions of the Benoit \& Saint-Aubin PDEs with particular asymptotic properties. Ann. Inst. Henri Poincaré D, to appear, 2018. Preprint: http://arxiv.org/abs/1605.06053

[PW17] E. Peltola and H. Wu. Global and local multiple SLEs for $\kappa \leq 4$ and connection probabilities for level lines of GFF. Preprint: http://arxiv.org/abs/1703.00898 2017.

[RRRA91] C. Ramirez, H. Ruegg, and M. Ruiz-Altaba. The contour picture of quantum groups in conformal field theories. Nucl. Phys., B364(1):195-233, 1991.

[Sch00] O. Schramm. Scaling limits of loop-erased random walks and uniform spanning trees. Israel J. Math., 118(1):221-288, 2000.

[Sel44] A. Selberg. Bemerkninger om et multiplet integral. Norsk Mat. Tidsskr., 26:71-78, 1944.

[Smi01] S. Smirnov. Critical percolation in the plane: conformal invariance, Cardy's formula, scaling limits. $C . R$. Acad. Sci. Paris, 333(3):239-244, 2001. See also http://arxiv.org/abs/0909.4499

[Var92] A. Varchenko. Multidimensional hypergeometric functions in conformal field theory, algebraic K-theory, algebraic geometry. In Proceedings of the International Congress of Mathematicians, Kyoto 1990, 1992.

[Var95] A. Varchenko. Multidimensional hypergeometric functions and representation theory of Lie algebras and quantum groups. Advanced Series in Mathematical Physics, Vol. 21. World Scientific, 1995.

[Zha08] D. Zhan. The scaling limits of planar LERW in finitely connected domains. Ann. Probab., 36(2):467-529, 2008. 